\title{
Poisson-Lie interpretation of trigonometric Ruijsenaars duality
}

\author{
L. Fehér ${ }^{a}$ and C. Klimčík ${ }^{b}$ \\ ${ }^{a}$ Department of Theoretical Physics, MTA KFKI RMKI \\ 1525 Budapest 114, P.O.B. 49, Hungary, and \\ Department of Theoretical Physics, University of Szeged \\ Tisza Lajos krt 84-86, H-6720 Szeged, Hungary \\ e-mail: lfeher@rmki.kfki.hu \\ ${ }^{b}$ Institut de mathématiques de Luminy, \\ 163, Avenue de Luminy, \\ 13288 Marseille, France \\ e-mail: klimcik@iml.univ-mrs.fr
}

\begin{abstract}
A geometric interpretation of the duality between two real forms of the complex trigonometric Ruijsenaars-Schneider system is presented. The phase spaces of the systems in duality are viewed as two different models of the same reduced phase space arising from a suitable symplectic reduction of the standard Heisenberg double of $U(n)$. The collections of commuting Hamiltonians of the systems in duality are shown to descend from two families of 'free' Hamiltonians on the double which are dual to each other in a Poisson-Lie sense. Our results give rise to a major simplification of Ruijsenaars' proof of the crucial symplectomorphism property of the duality map.
\end{abstract}




\section{Introduction}

In 1986 Ruijsenaars and Schneider [32] introduced a remarkable deformation of the nonrelativistic integrable many-body systems due to Calogero [3], Sutherland [34] and Moser [22]. The deformation corresponds to a passage from Galilei to Poincare invariance, and for this reason the deformed systems can be called relativistic Calogero systems. The family of Calogero type systems is very important both from the physical and from the mathematical point of view, and has been the subject of intense studies ever since its inception. See, e.g., the reviews [31, 35, 8].

When constructing action-angle maps for the classical, non-elliptic $A_{n-1}$ systems, Ruijsenaars [27, 29, 30] discovered an intriguing relation that arranges the Calogero type systems into 'dual pairs'. The main feature of the duality between system (i) and system (ii) is the fact that the action variables of system (i) are the particle-position variables of system (ii), and vice versa. The simplest example is provided by the non-relativistic rational Calogero system, whose self-duality was already noticed by Kazhdan, Kostant, and Sternberg [16] when treating the system by symplectic reduction of $T^{*} u(n) \simeq u(n) \times u(n)$, and thereby relating the symmetry between the $u(n)$ factors to the self-duality property. In his papers Ruijsenaars hinted at the possibility that there might exist an analogous geometric picture behind the duality in the other cases, too, leaving this as a problem for future investigation.

Later Gorsky and Nekrasov [15] and their coworkers [13, 14, 23] introduced new ideas and conjectures in the area of Ruijsenaars' duality. By generalizing [16], they proposed to interpret the duality in general in terms of symplectic reduction of two distinguished families of commuting Hamiltonians living on a suitable higher dimensional phase space. Upon a single reduction of this phase space described using two alternative gauge slices, i.e., two alternative models of the reduced phase space, those two families of commuting Hamiltonians may reduce to the two respective sets of action variables of the mutually dual systems. They mainly focused on reductions of infinite-dimensional phase spaces aiming to relate the Calogero type manybody systems to field theories. We wish to stress that also relatively simple finite-dimensional phase spaces can be considered for which these ideas work fully as expected. For example, we worked out in [10] the duality between the hyperbolic Sutherland and the rational RuijsenaarsSchneider systems [27] by reducing the cotangent bundle of the group $G L(n, \mathbb{C})$.

The key problem in the reduction approach is to find for each particular case of Ruijsenaars' duality two distinguished families of commuting Hamiltonians on an appropriate higherdimensional phase space and to find a way how to reduce those families. In the present paper, we solve this problem for one of the structurally most interesting and technically most involved cases of the duality that links together two particular real forms of the complex trigonometric Ruijsenaars-Schneider system [30]. The first real form is the original trigonometric RuijsenaarsSchneider system [32] characterized by the Lax matrix $L$ and symplectic form $\omega$

$$
\begin{gathered}
L_{j, k}(q, p)=\frac{e^{p_{k}} \sinh \left(\frac{x}{2}\right)}{\sinh \left(\mathrm{i} q_{j}-\mathrm{i} q_{k}+\frac{x}{2}\right)} \prod_{m \neq j}\left[1+\frac{\sinh ^{2} \frac{x}{2}}{\sin ^{2}\left(q_{j}-q_{m}\right)}\right]^{\frac{1}{4}} \prod_{m \neq k}\left[1+\frac{\sinh ^{2} \frac{x}{2}}{\sin ^{2}\left(q_{k}-q_{m}\right)}\right]^{\frac{1}{4}}, \\
\omega=\sum_{k=1}^{n} d p_{k} \wedge d q_{k}, \quad 0 \leq q_{k}<\pi, \quad q_{1}>q_{2}>\ldots>q_{n}, \quad p_{k} \in \mathbb{R},
\end{gathered}
$$


and the other real form is the Ruijsenaars dual of (1.1-2) that can be locally characterized by the Lax matrix $\hat{L}$ and symplectic form $\hat{\omega}$

$$
\begin{aligned}
\hat{L}_{j, k}(\hat{q}, \hat{p}) & =\frac{e^{\mathrm{i} \hat{q}_{k}} \sinh \left(-\frac{x}{2}\right)}{\sinh \left(\hat{p}_{j}-\hat{p}_{k}-\frac{x}{2}\right)} \prod_{m \neq j}\left[1-\frac{\sinh ^{2} \frac{x}{2}}{\sinh ^{2}\left(\hat{p}_{j}-\hat{p}_{m}\right)}\right]^{\frac{1}{4}} \prod_{m \neq k}\left[1-\frac{\sinh ^{2} \frac{x}{2}}{\sinh ^{2}\left(\hat{p}_{k}-\hat{p}_{m}\right)}\right]^{\frac{1}{4}}, \\
\hat{\omega} & =\sum_{k=1}^{n} d \hat{p}_{k} \wedge d \hat{q}_{k}, \quad 0 \leq \hat{q}_{k}<2 \pi, \quad \hat{p}_{j}-\hat{p}_{j+1}>\frac{|x|}{2} \quad(j=1, \ldots, n-1) .
\end{aligned}
$$

The Lax matrices are generating functions for Hamiltonians in involution. The 'main Hamiltonians' from which these systems derive their names read

$$
H_{\mathrm{RS}}(q, p)=\frac{1}{2} \operatorname{tr}\left(L(q, p)+L(q, p)^{-1}\right)=\sum_{j=1}^{n}\left(\cosh p_{j}\right) \prod_{m \neq j}\left[1+\frac{\sinh ^{2} \frac{x}{2}}{\sin ^{2}\left(q_{j}-q_{m}\right)}\right]^{\frac{1}{2}}
$$

and

$$
\hat{H}_{\mathrm{RS}}(\hat{q}, \hat{p})=\frac{1}{2} \operatorname{tr}\left(\hat{L}(\hat{q}, \hat{p})+\hat{L}(\hat{q}, \hat{p})^{-1}\right)=\sum_{j=1}^{n}\left(\cos \hat{q}_{j}\right) \prod_{m \neq j}\left[1-\frac{\sinh ^{2} \frac{x}{2}}{\sinh ^{2}\left(\hat{p}_{j}-\hat{p}_{m}\right)}\right]^{\frac{1}{2}} .
$$

Here $x$ is a real, non-zero coupling constant and the 'velocity of light' has been set to unity. The variables $(q, p)$ and $(\hat{q}, \hat{p})$ provide one-to-one set theoretic parametrizations of the phase spaces $P$ and $\hat{P}$ of the original and the dual Ruijsenaars-Schneider systems, respectively. As manifolds, $P$ is the cotangent bundle of the configuration space consisting of unordered $n$ tuples of distinct points on the circle $U(1)$, which corresponds to $n$ indistinguishable particles moving on the circle, and $\hat{P}$ is an open submanifold of the cotangent bundle of the torus $\mathbb{T}_{n}=U(1)^{\times n}$. In what follows $(P, \omega, L)$ denotes the collection of the Ruijsenaars-Schneider Hamiltonian systems defined by the spectral-invariants of $L(1.1)$, and similarly for $(\hat{P}, \hat{\omega}, \hat{L})$.

The duality between the real forms (1.1-2) and (1.3-4) was previously studied in [30] by the 'direct' approach, or, in other words, by an approach that did not use the methods of symplectic reduction. In particular, Ruijsenaars presented integration algorithms for the Hamiltonian flows of the original and dual systems and proved that the flows of the original system are complete on $(P, \omega)$ but the flows of the dual system are not complete on $(\hat{P}, \hat{\omega})$. He also pointed out that this singular behaviour of the dual system is related to the fact that the injective actionangle map from $(\hat{P}, \hat{\omega})$ into $(P, \omega)$ is not surjective but has only a dense open image. He then introduced an extension $\left(\hat{P}_{c}, \hat{\omega}_{c}\right)$ of the phase space $(\hat{P}, \hat{\omega})$ in order to achieve bijectivity of the corresponding extension of the action-angle map (alias the duality map) 1 . Remarkably, the dual flows turned out to be complete on the extended phase space $\left(\hat{P}_{c}, \hat{\omega}_{c}\right)$, which can be therefore referred to as the completion of $(\hat{P}, \hat{\omega})$.

Our interest in the real forms of the complex trigonometric Ruijsenaars-Schneider system was inspired by a conjecture that Gorsky and Nekrasov raised in [15]. They derived yet another trigonometric real form (called the $\mathrm{III}_{\mathrm{b}}$ system in [30]) from gauged WZW theory, and

\footnotetext{
${ }^{1}$ Our notations $P, \hat{P}, \hat{P}_{c}$ correspond, respectively, to $\Omega, \hat{\Omega}, \hat{\Omega} \sharp$ in [30]. Further notational correspondence is given in footnotes 4 and 5 in the text.
} 
conjectured that it should be possible to derive the same system also from an appropriate finitedimensional Heisenberg double. Although, as it stands, this conjecture remains still open, we recently succeeded to prove its 'extrapolation' to the trigonometric real form (1.1-2). Indeed, in our paper [11, we obtained the collection $(P, \omega, L)$ of the original Ruijsenaars-Schneider Hamiltonian systems by a reduction of a certain family of 'free' Hamiltonians on the Heisenberg double of the standard compact Poisson-Lie group $U(n)$. The 'free' Hamiltonians were constructed as the pull-backs of the dressing-invariant functions on the dual Poisson-Lie group $B(n)$ by the Iwasawa map $\Lambda_{R}$. (See Section 2.1 for the definitions of the Iwasawa maps and of the pertinent actions of $U(n)$.) Their basic properties are explicit integrability and invariance with respect to the so-called quasi-adjoint action of $U(n)$ on the double. Our present investigation relies on the existence of a dual family of 'free' Hamiltonians that have the same properties. In fact, the members of the dual family can be constructed by pulling-back to the double the conjugation-invariant functions on the Poisson-Lie group $U(n)$ by means of the dual Iwasawa map $\Xi_{R}$. As the main technical result of the present paper, we shall obtain the completion of the collection $(\hat{P}, \hat{\omega}, \hat{L})$ of the dual Ruijsenaars-Schneider Hamiltonian systems by reducing the dual family of free Hamiltonians on the Heisenberg double of $U(n)$. Notice that the first occurrence of the term 'dual' in the previous sentence refers to Ruijsenaars' duality of integrable systems and the second occurrence refers to duality in the sense of Poisson-Lie groups. Said in other words, the duality between the two trigonometric Ruijsenaars-Schneider systems described in [30] can be interpreted as a remnant of the 'geometric democracy' between the Poisson-Lie group $U(n)$ and the corresponding dual Poisson-Lie group $B(n)$ that survives the symplectic reduction. We believe that our results are related to the quantum group aspects of the standard trigonometric Ruijsenaars-Schneider system [28, 8, 24, 21] through the general correspondence between quantum groups and Poisson-Lie groups.

Both the original and the dual families of free Hamiltonian systems on the Heisenberg double are Poisson-Lie symmetric with respect to the quasi-adjoint action of $U(n)$ on the double. A decisive step in our work [11] was the choice of a suitable value $\nu(x)$ of the Poisson-Lie moment map $\Lambda$ of this quasi-adjoint symmetry such that the original Ruijsenaars-Schneider phase space $(P, \omega)$ could be identified with the constraint-manifold $F_{\nu(x)}:=\Lambda^{-1}(\nu(x))$ factorized by the gauge group given by the isotropy group $G_{\nu(x)}<U(n)$. In other words, $(P, \omega)$ was identified in [11] with the reduced phase space arising from the symplectic reduction of the Heisenberg double by the quasi-adjoint Poisson-Lie symmetry at the value $\nu(x)$ of the moment map $\Lambda$. In this paper, we shall demonstrate that the completion $\left(\hat{P}_{c}, \hat{\omega}_{c}\right)$ of the dual phase space $(\hat{P}, \hat{\omega})$ is also symplectomorphic to the same reduced phase space $F_{\nu}(x) / G_{\nu}(x)$. Therefore we can view $(P, \omega)$ and $\left(\hat{P}_{c}, \hat{\omega}_{c}\right)$ as two distinct models of a single reduced phase space. Moreover, as was already mentioned, we shall interpret the two collections of commuting Hamiltonians associated with the dual pair of Ruijsenaars-Schneider systems as reductions of two commutative families of free Hamiltonians on the Heisenberg double. Our results thus fit the geometric interpretation of Ruijsenaars' duality advocated by Gorsky and his collaborators for example in [13].

Speaking generally, the symplectic reduction approach often represents useful technical streamlining or simplification with respect to direct methods. This feature occurs also in our particular case. For instance, the free Hamiltonian systems on the Heisenberg double are themselves integrable and, as we shall see, their very simple Lax matrices reduce to the relatively complicated Lax matrices of the Ruijsenaars-Schneider systems. In addition, we can recover 
the integration algorithms for the original and dual Ruijsenaars-Schneider flows [30] simply by projecting the 'free' flows on the respective models of the reduced phase space $F_{\nu(x)} / G_{\nu(x)}$, and these projected flows are automatically complete. However, there is one aspect of the duality story where the reduction approach yields more than technical streamlining or simplification and, in fact, represents a major technical advantage with respect to the direct approach. Indeed, in the direct approach it was very difficult to prove the crucial symplectomorphism property of the extended action-angle map between the phase space $(P, \omega)$ and the extended dual phase space $\left(\hat{P}_{c}, \hat{\omega}_{c}\right)$. To realize the difficulties, the reader may consult the 'hyperbolic proof' published in [27] and its 'trigonometric analytic continuation' presented in [30]. In these references, a sophisticated web of non-trivial steps had been used combining scattering theory with demanding analysis and intricate analytic continuation arguments. However, from the point of view of the reduction approach the extended action-angle map is automatically a symplectomorphism, since it is easily recognized to be the composition of the symplectomorhism relating $(P, \omega)$ with $F_{\nu(x)} / G_{\nu(x)}$ and of the symplectomorphism relating $F_{\nu(x)} / G_{\nu(x)}$ with $\left(\hat{P}_{c}, \hat{\omega}_{c}\right)$.

To render justice to the direct methods, we note that so far considerably more examples of the duality were thoroughly investigated in the direct approach [27, 29, 30] than in the symplectic reduction approach. We hope, however, that the attractive features mentioned above supply sufficient motivation to further develop the reduction approach, along the lines discussed at the end of this article.

The rest of the paper is organized as follows. In Section 2, we first review the geometry of the standard Heisenberg double of $U(n)$ and recall the definition of the quasi-adjoint action of $U(n)$ on it. We then describe the two families of free Hamiltonian systems on the double that are Poisson-Lie symmetric with respect to the quasi-adjoint action. We do not claim any new results in this section, although we could not find in the literature any previous detailed treatment of the free flows as given by our Propositions 2.1 and 2.2.

The reduction of the two families of free Hamiltonians to the respective two real forms of the complex trigonometric Ruijsenaars-Schneider system is presented in Section 3. After a summary of required notions, Theorems 3.1 and 3.2 state in a strengthened form the result of [11], where the first family was reduced to the system $(P, \omega, L)$. Then the new Theorems 3.3 and 3.4 are formulated, which claim that the same symplectic reduction applied to the second family yields the completion of the system $(\hat{P}, \hat{\omega}, \hat{L})$. Section 4 is devoted to the proofs of Theorems 3.1 and 3.2. Although many results described in this section were obtained already in our letter [11], we still add here important new material. Namely, we pay special attention to the Weyl group covering of the phase space $P$ of the original Ruijsenaars-Schneider system, since this enables us to recover the geometric meaning of the standard coordinates on $P$ from the reduction. Indeed, in [11, we identified the Ruijsenaars-Schneider system with the reduced system on $F_{\nu(x)} / G_{\nu(x)}$ by means of a somewhat mysterious coordinate transformation that was just 'cooked up' to do the job. Here we explain the geometrical origin of this transformation.

Theorems 3.3 and 3.4 represent the main results obtained in this paper and their proof occupies much of Section 5. As a by-product, the integration algorithm for the dual flows is also treated at the end of Section 5. Further discussion, including comparison with [30], is offered in Section 6. Finally, Appendix A contains an alternative proof of the fact that $F_{\nu(x)}$ is an embedded submanifold of the Heisenberg double, and Appendix B is devoted to the subtle topology of the configuration space of $n$ indistinguishable point-particles moving on the circle. 


\section{Free systems on the Heisenberg double}

Let us recall that a Poisson-Lie group is a Lie group, $G$, equipped with a Poisson bracket, $\{., .\}_{G}$, such that the multiplication map $G \times G \rightarrow G$ is Poisson. It is an important concept that makes it possible to generalize the usual notion of symmetry for Hamiltonian systems. Namely, if the Poisson-Lie group $G$ acts on a symplectic manifold $M$ in a Poisson way (i.e. the action map $G \times M \rightarrow M$ is Poisson) and, moreover, if the Hamiltonian $H$ is $G$-invariant, then one says that the system $(M, H)$ is $G$ Poisson-Lie symmetric 33. For our purpose we shall focus on the group $G=U(n)$ equipped with its standard Poisson-Lie structure, although all results collected in this section hold true, with minor modifications, for any compact reductive group $G$.

In subsection 2.1, we summarize the necessary information concerning the structure of the Heisenberg double of the dual pair of Poisson-Lie groups $U(n)$ and $B(n)$ and also recall the notion of the quasi-adjoint action of $U(n)$ on the double. Then, in subsection 2.2, we describe the two families of $U(n)$ Poisson-Lie symmetric Hamiltonian systems that will descend upon symplectic reduction to the trigonometric Ruijsenaars-Schneider system (1.1) and its dual. These systems turn out to be explicitly integrable, and for this reason we call the underlying Hamiltonians 'free' Hamiltonians.

\subsection{Recall of the Heisenberg double and the quasi-adjoint action}

The Heisenberg double is a Poisson manifold $\left(D,\{., .\}_{+}\right)$that Semenov-Tian-Shansky [33] associated with any Poisson-Lie group $\left(G,\{., .\}_{G}\right)$. The manifold $D$ is itself a Lie group, the Drinfeld double of $G$, and its Poisson bracket $\{., .\}_{+}$can be expressed in terms of the factorizable $r$-matrix of Lie $(D)$. One can also directly define the double $\left(D,\{., .\}_{+}\right)$and then recover from it the Poisson-Lie group $\left(G,\{., .\}_{G}\right)$ and its dual.

Consider the real Lie group $D:=G L(n, \mathbb{C})$ and endow the corresponding real Lie algebra $\mathcal{D}:=g l(n, \mathbb{C})$ with the non-degenerate, invariant 'scalar product'

$$
(X, Y)_{\mathcal{D}}:=\Im \operatorname{tr}(X Y), \quad \forall X, Y \in \mathcal{D} .
$$

Here $\Im z$ stands for the imaginary part of the complex number $z$. Let $B:=B(n)$ be the subgroup of $D$ formed by the upper-triangular matrices having positive entries along the diagonal, and denote $G:=U(n)$. As a real vector space, we have the direct sum decomposition

$$
\mathcal{D}=\mathcal{G}+\mathcal{B},
$$

where $\mathcal{G}:=\operatorname{Lie}(G)=u(n)$ and $\mathcal{B}:=\operatorname{Lie}(B)$ are isotropic subalgebras mutually dual to each other with respect to the pairing provided by $(., .)_{\mathcal{D}}$. In other words, we have a so-called Manin triple in our hands, and thus the real Lie group $D$ carries two natural Poisson structures $\{., .\}_{ \pm}$. Here we need only the structure $\{., .\}_{+}$, and to define it we introduce the projection operators $\pi_{\mathcal{G}}: \mathcal{D} \rightarrow \mathcal{G}$ and $\pi_{\mathcal{B}}: \mathcal{D} \rightarrow \mathcal{B}$ associated with the splitting (2.2). Furthermore, for any real function $\Phi \in C^{\infty}(D)$ introduce the left- and right gradients $\nabla^{L, R} \Phi \in C^{\infty}(D, \mathcal{D})$ by

$$
\left.\frac{d}{d s}\right|_{s=0} \Phi\left(e^{s X} K e^{s Y}\right)=\left(X, \nabla^{L} \Phi(K)\right)_{\mathcal{D}}+\left(Y, \nabla^{R} \Phi(K)\right)_{\mathcal{D}}, \quad \forall X, Y \in \mathcal{D}, \forall K \in D .
$$


By using the factorizable $r$-matrix of $\mathcal{D}$,

$$
\rho:=\frac{1}{2}\left(\pi_{\mathcal{G}}-\pi_{\mathcal{B}}\right)
$$

for any $\Phi_{1}, \Phi_{2} \in C^{\infty}(D)$ one has the Poisson bracket

$$
\left\{\Phi_{1}, \Phi_{2}\right\}_{+}(K)=\left(\nabla^{R} \Phi_{1}(K), \rho\left(\nabla^{R} \Phi_{2}(K)\right)\right)_{\mathcal{D}}+\left(\nabla^{L} \Phi_{1}(K), \rho\left(\nabla^{L} \Phi_{2}(K)\right)\right)_{\mathcal{D}} .
$$

The Poisson manifold $\left(D,\{., .\}_{+}\right)$is called the Heisenberg double of $G$.

By the Iwasawa decomposition, each element $K \in D$ has the unique representations

$$
K=b_{L} g_{R}^{-1} \quad \text { and } \quad K=g_{L} b_{R}^{-1} \quad \text { with } \quad b_{L, R} \in B, g_{L, R} \in G .
$$

As a result of the global character of this decomposition, our $\left(D,\{.,\}_{+}\right)$is actually symplectic. The underlying symplectic form, called $\omega_{+}$, was found by Alekseev and Malkin [1]. In fact, with the help of the Iwasawa maps $\Lambda_{L, R}: D \rightarrow B$ and $\Xi_{L, R}: D \rightarrow G$ defined by using (2.6) as

$$
\Lambda_{L, R}(K):=b_{L, R} \quad \text { and } \quad \Xi_{L, R}(K):=g_{L, R}
$$

one has

$$
\omega_{+}=\frac{1}{2} \Im \operatorname{tr}\left(d \Lambda_{L} \Lambda_{L}^{-1} \wedge d \Xi_{L} \Xi_{L}^{-1}\right)+\frac{1}{2} \Im \operatorname{tr}\left(d \Lambda_{R} \Lambda_{R}^{-1} \wedge d \Xi_{R} \Xi_{R}^{-1}\right) .
$$

Having described the Heisenberg double, next we recall how the Poisson bracket $\{., .\}_{+}$ induces Poisson-Lie structures on the groups $B$ and $G$. As a preparation, define the left- and right derivatives $d^{L, R} f \in C^{\infty}(B, \mathcal{G})$ for a real function $f \in C^{\infty}(B)$ by the equality

$$
\left.\frac{d}{d s}\right|_{s=0} f\left(e^{s X} b e^{s Y}\right)=\left(X, d^{L} f(b)\right)_{\mathcal{D}}+\left(Y, d^{R} f(b)\right)_{\mathcal{D}}, \quad \forall X, Y \in \mathcal{B}, \forall b \in B .
$$

For a real function $\phi \in C^{\infty}(G)$ define $d^{L, R} \phi \in C^{\infty}(G, \mathcal{B})$ similarly,

$$
\left.\frac{d}{d s}\right|_{s=0} \phi\left(e^{s X} g e^{s Y}\right)=\left(X, d^{L} \phi(g)\right)_{\mathcal{D}}+\left(Y, d^{R} \phi(g)\right)_{\mathcal{D}}, \quad \forall X, Y \in \mathcal{G}, \forall g \in G .
$$

By using the negative-definite scalar product on $\mathcal{G}$ furnished by

$$
\langle X, Y\rangle_{\mathcal{G}}:=\operatorname{tr}(X Y), \quad \forall X, Y \in \mathcal{G},
$$

introduce also $\mathrm{D}^{L, R} \phi \in C^{\infty}(G, \mathcal{G})$ by

$$
\left.\frac{d}{d s}\right|_{s=0} \phi\left(e^{s X} g e^{s Y}\right)=\left\langle X, \mathbf{D}^{L} \phi(g)\right\rangle_{\mathcal{G}}+\left\langle Y, \mathbf{D}^{R} \phi(g)\right\rangle_{\mathcal{G}}, \quad \forall X, Y \in \mathcal{G}, \forall g \in G .
$$

Defining $R^{\mathrm{i}} \in \operatorname{End}(\mathcal{G})$ as

$$
R^{\mathrm{i}}(X)=\pi_{\mathcal{G}}(-\mathrm{i} X), \quad \forall X \in \mathcal{G},
$$

which is actually nothing but the standard $r$-matrix of $\mathcal{G}$, it is easy to check that the two types of derivatives over $G$ are related by

$$
d^{L} \phi=\mathrm{i} \mathbf{D}^{L} \phi+R^{\mathrm{i}}\left(\mathbf{D}^{L} \phi\right), \quad d^{R} \phi=\mathrm{i} \mathbf{D}^{R} \phi+R^{\mathrm{i}}\left(\mathbf{D}^{R} \phi\right),
$$


which in particular implies that

$$
d^{L, R} \phi=\pi_{\mathcal{B}}\left(\mathrm{i} \mathbf{D}^{L, R} \phi\right), \quad \forall \phi \in C^{\infty}(G)
$$

After fixing all the above notations, we are ready to write down explicit formulas for the Poisson-Lie structures $\{., .\}_{B}$ on $B$ and $\{., .\}_{G}$ on $G$. In fact, it can be shown that the algebras of functions $C^{\infty}(B)$ and $C^{\infty}(G)$ pulled-back, respectively, by the Iwasawa maps $\Lambda_{R}$ and $\Xi_{R}$ form two Poisson subalgebras of $C^{\infty}(D)$. Because of the surjectivity of the Iwasawa maps in (2.7), the bracket $\{., .\}_{+}$on $D$ thus induces two brackets $\{., .\}_{B}$ and $\{., .\}_{G}$ by the following prescriptions:

$$
\left\{\Lambda_{R}^{*} f_{1}, \Lambda_{R}^{*} f_{2}\right\}_{+}=\Lambda_{R}^{*}\left\{f_{1}, f_{2}\right\}_{B} \quad \text { and } \quad\left\{\Xi_{R}^{*} \phi_{1}, \Xi_{R}^{*} \phi_{2}\right\}_{+}=\Xi_{R}^{*}\left\{\phi_{1}, \phi_{2}\right\}_{G} .
$$

The brackets $\{., .\}_{B}$ and $\{., .\}_{G}$ induced in this way are just the Poisson-Lie brackets on $B$ and on $G$, respectively. The explicit form of the induced Poisson bracket on $B$ reads

$$
\left\{f_{1}, f_{2}\right\}_{B}(b)=-\left(b^{-1}\left(d^{L} f_{1}(b)\right) b, d^{R} f_{2}(b)\right)_{\mathcal{D}}, \quad \forall f_{1}, f_{2} \in C^{\infty}(B), \forall b \in B .
$$

This is obtained from (2.5) and (2.6) using that if $\Phi=\Lambda_{R}^{*} f$ for some $f \in C^{\infty}(B)$, then

$$
\nabla^{L} \Phi(K)=-g_{L}\left(d^{R} f\left(b_{R}\right)\right) g_{L}^{-1}, \quad \nabla^{R} \Phi(K)=-b_{R}\left(d^{R} f\left(b_{R}\right)\right) b_{R}^{-1} .
$$

The induced Poisson-Lie structure on $G$ permits the analogous formula

$$
\left\{\phi_{1}, \phi_{2}\right\}_{G}(g)=\left(g^{-1}\left(d^{L} \phi_{1}(g)\right) g, d^{R} \phi_{2}(g)\right)_{\mathcal{D}}, \quad \forall \phi_{1}, \phi_{2} \in C^{\infty}(G), \forall g \in G,
$$

which can be conveniently rewritten as

$$
\left\{\phi_{1}, \phi_{2}\right\}_{G}(g)=\left\langle\mathbf{D}^{R} \phi_{1}(g), R^{\mathrm{i}}\left(\mathbf{D}^{R} \phi_{2}(g)\right)\right\rangle_{\mathcal{G}}-\left\langle\mathbf{D}^{L} \phi_{1}(g), R^{\mathrm{i}}\left(\mathbf{D}^{L} \phi_{2}(g)\right)\right\rangle_{\mathcal{G}}
$$

by virtue of (2.15) $)$. In the last formula only $G$ features explicitly, while (2.17) and (2.19) are formulated relying on conjugations defined in the group $D$.

The Poisson bracket $\{., .\}_{+}$closes also on $\Lambda_{L}^{*} C^{\infty}(B)$ and on $\Xi_{L}^{*} C^{\infty}(G)$. In fact, by using $\Lambda_{L}$ and $\Xi_{L}$ (2.7) one obtains the same Poisson-Lie structures on $B$ and on $G$ as by means of (2.16). Another important fact is that the elements of $\Lambda_{R}^{*} C^{\infty}(B)$ (respectively $\Xi_{R}^{*} C^{\infty}(G)$ ) commute with the elements of $\Lambda_{L}^{*} C^{\infty}(B)$ (respectively $\Xi_{L}^{*} C^{\infty}(G)$ ) with respect to $\{., .\}_{+}$.

Finally, we recall from [18] the so-called quasi-adjoint Poisson action of $G$ on $\left(D, \omega_{+}\right)$. The corresponding action map $G \times D \rightarrow D$ sends $(g, K)$ to $g \triangleright K$ defined by

$$
g \triangleright K:=g K \Xi_{R}\left(g \Lambda_{L}(K)\right), \quad \forall g \in G, \forall K \in D .
$$

The composition property, $g_{1} \triangleright\left(g_{2} \triangleright K\right)=\left(g_{1} g_{2}\right) \triangleright K$ for all $g_{1}, g_{2} \in G$ and $K \in D$, can be checked by using (2.6) and (2.7). The $G$-action $\triangleright$ admits the equivariant Poisson-Lie moment map $\Lambda: D \rightarrow B$ given by

$$
\Lambda(K)=\Lambda_{L}(K) \Lambda_{R}(K), \quad \forall K \in D
$$


This means that $\Lambda$ enjoys the following two properties. First,

$$
X_{D}[\Phi]=\left(X,\{\Phi, \Lambda\}_{+} \Lambda^{-1}\right)_{\mathcal{D}}, \quad \forall X \in \mathcal{G}, \forall \Phi \in C^{\infty}(D),
$$

where $\left(X_{D}[\Phi]\right)(K):=\left.\frac{d}{d s} \Phi\left(e^{s X} \triangleright K\right)\right|_{s=0}$ for all $K \in D$. Second,

$$
\Lambda(g \triangleright K)=\operatorname{Dress}_{g}(\Lambda(K)), \quad \forall g \in G, \forall K \in D,
$$

where we use the dressing action of $G$ on $B$ that operates as

$$
\operatorname{Dress}_{g}(b):=\Lambda_{L}(g b), \quad \forall g \in G, \forall b \in B .
$$

The quasi-adjoint action (2.21) was found in [18] by the following method. One first observes that the map $\Lambda$ defined by (2.22) satisfies (2.24) and it generates via (2.23) an infinitesimal action of $\mathcal{G}$ on $D$. These statements are implied [20] by the fact that $\Lambda: D \rightarrow B$ is a Poisson map, which is obvious. The resulting infinitesimal action was then integrated, and the $G$-action obtained in this way automatically has the Poisson property, i.e., the action map $G \times D \rightarrow D$ is a Poisson map.

Remark 2.1. It follows from (2.21) that, like for the ordinary adjoint action, the central $U(1)$ subgroup of $G=U(n)$ acts trivially, and the factor group $U(n) / U(1)$ acts effectively. It is also easy to check (see Appendix A) that the map $\Lambda$ (2.22) takes its values in the Poisson-Lie subgroup $S B$ of $B$ consisting of the elements of determinant one. Consider the projection

$$
\pi_{D}: D \rightarrow \bar{D}:=D / G L(1, \mathbb{C})
$$

where $G L(1, \mathbb{C})$ denotes the center of $D=G L(n, \mathbb{C})$, and define subgroups of $\bar{D}$ by

$$
\bar{G}:=\pi_{D}(G) \quad \text { and } \quad \bar{B}:=\pi_{D}(B)=\pi_{D}(S B)
$$

The groups $\bar{G}$ and $\bar{B}$ sit in $\bar{D}$ in quite the same way as $G$ and $B$ sit in $D$, and therefore are dual to each other in the Poisson-Lie sense. In fact, $\bar{D}$ carries a symplectic structure inherited from $\left(D, \omega_{+}\right)$, whereby it can be regarded as the Heisenberg double constituted by $\bar{G}$ and $\bar{B}$. The projection $\pi_{D}$ gives rise to natural isomorphisms

$$
U(n) / U(1) \simeq \bar{G} \quad \text { and } \quad S B \simeq \bar{B} .
$$

By using these identifications, the map $\Lambda: D \rightarrow S B$ given by (2.22) yields the Poisson-Lie moment map for the quasi-adjoint action of $U(n) / U(1)$ on $D$.

\subsection{Integration algorithms for the free flows}

Our principal aim now is to present the flows of two families of commuting Hamiltonians on the Heisenberg double that are invariant with respect to the quasi-adjoint action of $G$.

The Hamiltonians of our interest form the rings $\mathcal{H}$ and $\hat{\mathcal{H}}$ defined by

$$
\mathcal{H}:=\Lambda_{R}^{*} C^{\infty}(B)^{c} \quad \text { and } \quad \hat{\mathcal{H}}:=\Xi_{R}^{*} C^{\infty}(G)^{G} .
$$


Here $C^{\infty}(B)^{c}$ denotes the center of the Poisson-Lie structure on $C^{\infty}(B)$ and $C^{\infty}(G)^{G}$ contains the functions on $G$ that are invariant with respect to the standard adjoint action of $G$ on $G$. It is obvious that all elements of $\mathcal{H}$ mutually Poisson commute which each other. The Poisson commutativity of $\hat{\mathcal{H}}$ also takes places, because $\mathbf{D}^{L} \phi=\mathbf{D}^{R} \phi$ for any $\phi \in C^{\infty}(G)^{G}$, and hence (2.20) implies that $\left\{\phi_{1}, \phi_{2}\right\}_{G}=0$ for any $\phi_{1}, \phi_{2} \in C^{\infty}(G)^{G}$. Note, however, that the elements of $C^{\infty}(G)^{G}$ do not lie in the center of the Poisson-Lie structure on $C^{\infty}(G)$ in general.

It is well known and is readily seen from (2.17) and (2.25) that

$$
C^{\infty}(B)^{c}=C^{\infty}(B)^{G}
$$

with the dressing-invariant functions on the right-hand side. Moreover, if inv : $B \rightarrow B$ is the (anti-Poisson) inversion map, then one can verify that inv ${ }^{*}$ stabilizes $C^{\infty}(B)^{c}$ and

$$
\Lambda_{R}^{*} f=\Lambda_{L}^{*}(f \circ \text { inv }), \quad \forall f \in C^{\infty}(B)^{c} .
$$

In particular, it follows that

$$
\mathcal{H}:=\Lambda_{R}^{*} C^{\infty}(B)^{c}=\Lambda_{L}^{*} C^{\infty}(B)^{c} .
$$

To obtain another useful characterization of $\mathcal{H}$, consider the diffeomorphism, $\mathfrak{P}$, from $B$ to the space of Hermitian positive definite matrices, $\mathcal{P}$, defined by

$$
\mathfrak{P}(b):=b b^{\dagger}, \quad \forall b \in B .
$$

The map $\mathfrak{P}$ intertwines the dressing action (2.25) on $B$ with the ordinary conjugation action on $\mathcal{P}$,

$$
\mathfrak{P}\left(\text { Dress }_{g} b\right)=g \mathfrak{P}(b) g^{-1}, \quad \forall g \in G, \forall b \in B,
$$

whereby the elements of $C^{\infty}(B)^{c}$ correspond to the spectral-invariants $C^{\infty}(\mathcal{P})^{G}$ on $\mathcal{P}$.

It is of crucial importance that all Hamiltonians in $\mathcal{H}$ and in $\hat{\mathcal{H}}$ are invariant under the quasi-adjoint action (2.21) on the double. This holds since for all $g \in G$ and $K \in D$ one has

$$
\Lambda_{L}(g \triangleright K)=\operatorname{Dress}_{g}\left(\Lambda_{L}(K)\right), \quad \Xi_{R}(g \triangleright K)=\Xi_{R}\left(g \Lambda_{L}(K)\right)^{-1} \Xi_{R}(K) \Xi_{R}\left(g \Lambda_{L}(K)\right) .
$$

The first relation and (2.30), (2.32) imply the $G$ invariance of the elements of $\mathcal{H}$, which also follows directly from $\Lambda_{R}(g \triangleright K)=\operatorname{Dress}_{\Xi_{R}\left(g \Lambda_{L}(K)\right)^{-1}}\left(\Lambda_{R}(K)\right)$.

Now we are ready to show that the evolution equations of the Hamiltonian systems $\left(D, \omega_{+}, H\right)$ and $\left(D, \omega_{+}, \hat{H}\right)$ can be explicitly solved for any $H \in \mathcal{H}$ and any $\hat{H} \in \hat{\mathcal{H}}$. We start with $\mathcal{H}$.

Proposition 2.1. The flow induced on the Heisenberg double $D$ by the Hamiltonian $H=\Lambda_{R}^{*} f$ with an arbitrary $f \in C^{\infty}(B)^{c}$ is given by

$$
K(t)=g_{L}(0) \exp \left[-t d^{R} f\left(b_{R}(0)\right)\right] b_{R}^{-1}(0) .
$$

In terms of the Iwasawa decompositions (2.6), the flow can be written as

$$
g_{L}(t)=g_{L}(0) \exp \left[-t d^{R} f\left(b_{R}(0)\right)\right], \quad b_{R}(t)=b_{R}(0),
$$




$$
g_{R}(t)=\exp \left[-t d^{L} f\left(b_{L}(0)\right)\right] g_{R}(0), \quad b_{L}(t)=b_{L}(0) .
$$

Proof. Equation (2.5) entails that the Hamiltonian vector field, $V_{\Phi}$, generated by an arbitrary $\Phi \in C^{\infty}(D)$ has the form

$$
V_{\Phi}(K)=K \rho\left(\nabla^{R} \Phi(K)\right)+\rho\left(\nabla^{L} \Phi(K)\right) K .
$$

Notice from (2.17) that

$$
\pi_{\mathcal{B}}\left(b\left(d^{R} f(b)\right) b^{-1}\right)=0, \quad \forall b \in B, \forall f \in C^{\infty}(B)^{c} .
$$

By combining these formulae and (2.18), we obtain

$$
V_{\Phi}(K)=-g_{L}\left(d^{R} f\left(b_{R}\right)\right) b_{R}^{-1}, \quad \text { if } \quad \Phi=\Lambda_{R}^{*} f, f \in C^{\infty}(B)^{c} .
$$

At the same time, since $\Lambda_{R}: D \rightarrow B$ is a Poisson map, we also have $V_{\Phi}\left(b_{R}\right)=0$. It follows immediately that the flow is given by (2.36), which obviously translates into (2.37). The alternative formula (2.38) can be established using that $b_{L}(t)=b_{L}(0)$ by (2.32), and that $d^{L} f(b)=b\left(d^{R} f(b)\right) b^{-1}$ for any $f \in C^{\infty}(B)^{c}$. Q.E.D.

Proposition 2.1 says that the Poisson-Lie momenta $b_{L}, b_{R}$ are constants of motion and both 'position-like' variables $g_{L}, g_{R}$ follow Killing geodesics on $G$. In a special case, this statement first appeared in [38].

Now we turn to the flows associated with the conjugation-invariant functions on $G$. We begin by treating this problem on the Poisson-Lie group $G$.

Lemma 2.1. For $\phi \in C^{\infty}(G)^{G}$, consider the Hamiltonian evolution equation on $\left(G,\{., .\}_{G}\right)$,

$$
\dot{g}:=\{g, \phi\}_{G}=\left[g, R^{\mathrm{i}}(\mathbf{D} \phi(g))\right],
$$

where we denote $\mathbf{D}^{L} \phi=\mathbf{D}^{R} \phi$ simply by $\mathbf{D} \phi$. Taking an arbitrary initial value $g(0)$, let the curves $\beta(t) \in B$ and $\gamma(t) \in G$ be the (unique, smooth) solutions of the factorization problem

$$
e^{\mathrm{i} t \mathbf{D} \phi(g(0))}=\beta(t) \gamma(t)
$$

Then the solution of (2.42) with the initial value $g(0)$ is given by

$$
g(t)=\gamma(t) g(0) \gamma(t)^{-1}
$$

Proof. By taking the derivative of (2.44), we obtain

$$
\dot{g}(t)=\left[\dot{\gamma}(t) \gamma(t)^{-1}, g(t)\right]
$$

On the other hand, by taking the derivative of (2.43) we obtain

$$
\beta(t) \gamma(t)(\mathrm{i} \mathbf{D} \phi(g(0)))=\dot{\beta}(t) \gamma(t)+\beta(t) \dot{\gamma}(t) .
$$


This implies

$$
\mathrm{i} \gamma(t) \mathbf{D} \phi(g(0)) \gamma(t)^{-1}=\mathrm{i} \mathbf{D} \phi(g(t))=\beta(t)^{-1} \dot{\beta}(t)+\dot{\gamma}(t) \gamma(t)^{-1},
$$

where the first equality follows from the invariance property of $\phi$. We see from (2.47) that

$$
\dot{\gamma}(t) \gamma(t)^{-1}=\pi_{\mathcal{G}}(\mathrm{i} \mathbf{D} \phi(g(t)))=-R^{\mathrm{i}}(\mathbf{D} \phi(g(t)),
$$

which concludes the proof. Q.E.D.

Proposition 2.2. The flow $K(t)=b_{L}(t) g_{R}(t)^{-1}$ induced on the Heisenberg double by the Hamiltonian $\hat{H}=\Xi_{R}^{*} \phi$ with an arbitrary $\phi \in C^{\infty}(G)^{G}$ is given by

$$
g_{R}(t)=\gamma(t) g_{R}(0) \gamma(t)^{-1}, \quad b_{L}(t)=b_{L}(0) \beta(t)
$$

in terms of the solutions $\gamma(t) \in G, \beta(t) \in B$ of the factorization problem $e^{\mathrm{i} t \mathbf{D} \phi\left(g_{R}(0)\right)}=\beta(t) \gamma(t)$.

Proof. Consider first an arbitrary 'collective Hamiltonian' on $D$ of the type

$$
\Phi=\Xi_{R}^{*} \phi, \quad \phi \in C^{\infty}(G) .
$$

In this case one obtains directly from the definitions that

$$
\nabla^{L} \Phi(K)=-b_{L}\left(d^{R} \phi\left(g_{R}\right)\right) b_{L}^{-1}, \quad \nabla^{R} \Phi(K)=-g_{R}\left(d^{R} \phi\left(g_{R}\right)\right) g_{R}^{-1} .
$$

With the aid of these relations, the evolution equation $\dot{K}=V_{\Phi}(K)(2.39)$ can be spelled out as

$$
\begin{aligned}
& \dot{g}_{R}=g_{R} R^{\mathrm{i}}\left(\mathbf{D}^{R} \phi\left(g_{R}\right)\right)-R^{\mathrm{i}}\left(\mathbf{D}^{L} \phi\left(g_{R}\right)\right) g_{R}, \\
& \dot{b}_{L}=b_{L}\left(d^{R} \phi\left(g_{R}\right)\right)=b_{L} \pi_{\mathcal{B}}\left(\mathrm{i}^{R} \phi\left(g_{R}\right)\right) .
\end{aligned}
$$

Besides (2.51), we also used (2.13) and (2.14) to get this system of equations. Notice that (2.52) is just the Hamiltonian evolution equation generated by $\phi$ on $\left(G,\{., .\}_{G}\right)$. If we now assume that $\phi \in C^{\infty}(G)^{G}$, then the desired solution of the last system of equations is easily found with the aid of (2.44) and (2.47). This yields the flow as claimed in (2.49). Q.E.D.

Corollary 2.1. The integral curve of the Hamiltonian $\hat{H}=\Xi_{R}^{*} \phi, \phi \in C^{\infty}(G)^{G}$ satisfies

$$
K(t) K^{\dagger}(t)=b_{L}(t) b_{L}(t)^{\dagger}=b_{L}(0) e^{2 \mathrm{i} t \mathbf{D} \phi\left(g_{R}(0)\right)} b_{L}(0)^{\dagger} .
$$

Proof. Combine (2.49) with $b_{L}(0) \beta(t)\left(b_{L}(0) \beta(t)\right)^{\dagger}=b_{L}(0) \beta(t) \gamma(t)\left(b_{L}(0) \beta(t) \gamma(t)\right)^{\dagger}$. Q.E.D.

We finish this section with a few comments. First we notice from the statement below (2.34) that $C^{\infty}(B)^{c}$ is functionally generated by the (not all independent) invariants

$$
f_{k}(b):=\frac{1}{2 k} \operatorname{tr}\left(b b^{\dagger}\right)^{k}, \quad \forall k \in \mathbb{Z}^{*}
$$

It is also clear that $C^{\infty}(G)^{G}$ is generated by the functions

$$
\phi_{k}(g):=\frac{1}{2 k} \operatorname{tr}\left(g^{k}+g^{-k}\right), \quad \phi_{-k}(g):=\frac{1}{2 k \mathrm{i}} \operatorname{tr}\left(g^{k}-g^{-k}\right), \quad \forall k \in \mathbb{Z}_{+} .
$$


The flows of the generators $H_{k}:=\Lambda_{R}^{*} f_{k}$ of $\mathcal{H}$ and $\hat{H}_{k}:=\Xi_{R}^{*} \phi_{k}$ of $\hat{\mathcal{H}}$ can be written down explicitly using that

$$
\begin{gathered}
d^{R} f_{k}(b)=\mathrm{i}\left(b^{\dagger} b\right)^{k}, \quad d^{L} f_{k}(b)=\mathrm{i}\left(b b^{\dagger}\right)^{k}, \quad \forall k \in \mathbb{Z}^{*}, \\
\mathbf{D} \phi_{k}(g)=\frac{1}{2}\left(g^{k}-g^{-k}\right), \quad \mathbf{D} \phi_{-k}(g)=\frac{1}{2 \mathrm{i}}\left(g^{k}+g^{-k}\right), \quad \forall k \in \mathbb{Z}_{+} .
\end{gathered}
$$

For later reference, we record that if one considers a real linear combination $\phi:=\sum_{k \neq 0} \mu_{k} \phi_{k}$, and writes it with some analytic function $\chi$ in the form $\phi(g)=\operatorname{tr}(\chi(g))+$ c.c., then one has

$$
\mathbf{D} \phi(g)=g \chi^{\prime}(g)-\left(g \chi^{\prime}(g)\right)^{\dagger} .
$$

We note from the above that the Hamiltonians $H_{k}$ and $\hat{H}_{k}$, and more generally the elements of the rings $\mathcal{H}$ and $\hat{\mathcal{H}}(2.29)$ that they generate, are spectral-invariants of the respective matrix functions $\mathcal{L}$ and $\hat{\mathcal{L}}$ on the Heisenberg double $D$ defined by

$$
\mathcal{L}: K \mapsto b_{R} b_{R}^{\dagger} \quad \text { and } \quad \hat{\mathcal{L}}: K \mapsto g_{R}
$$

with the Iwasawa decompositions in (2.6). For our purpose, it will be fruitful to view $\mathcal{L}$ and $\hat{\mathcal{L}}$ as 'unreduced Lax matrices' and the following convention will also prove to be very convenient.

Definition 2.1. By using the previous notations (2.29) and (2.60), we define the collections of Hamiltonian systems

$$
\begin{aligned}
& \left(D, \omega_{+}, \mathcal{L}\right):=\left\{\left(D, \omega_{+}, H\right) \mid H \in \mathcal{H}\right\}, \\
& \left(D, \omega_{+}, \hat{\mathcal{L}}\right):=\left\{\left(D, \omega_{+}, \hat{H}\right) \mid \hat{H} \in \hat{\mathcal{H}}\right\},
\end{aligned}
$$

and henceforth refer to these collections as the 'canonical free systems'.

Any member of the above collections is integrable in the obvious sense that one can directly write down its Hamiltonian flow, as given by Propositions 2.1 and 2.2. In the rest of the paper we shall study the symplectic reduction of the canonical free systems, i.e., the simultaneous reduction of all the Hamiltonian systems that constitute them. The main advantage of our, somewhat non-standard, notation (2.61) is that it suggests that one should directly reduce the Lax matrices $\mathcal{L}$ and $\hat{\mathcal{L}}$, instead of separately reducing the Hamiltonians that they generate. In this respect, observe from (2.35) and the sentence afterwards that the quasi-adjoint action (2.21) operates by similarity transformations on $\mathcal{L}$ and on $\hat{\mathcal{L}}$ in (2.60). Since, as generators of commuting Hamiltonians, any two Lax matrices that are related by a similarity transformation are equivalent, one can indeed use the quasi-adjoint action to reduce the (equivalence classes of the) Lax matrices $\mathcal{L}$ and $\hat{\mathcal{L}}$. The usefulness of this point of view will become clear in Section 3 .

Finally, for clarity, let us remark that the elements of the Abelian subalgebra $\Xi_{L}^{*} C^{\infty}(G)^{G}$ of the Poisson algebra $C^{\infty}(D)$ are in general not invariant under the quasi-adjoint action (2.21). Indeed

$$
\Xi_{L}(g \triangleright K)=g \Xi_{L}(K) \Xi_{L}\left(\Lambda_{R}(K)^{-1} \Xi_{R}\left(g \Lambda_{L}(K)\right)\right)
$$

is not conjugate to $\Xi_{L}(K)$ in general. However, the elements of $\Xi_{L}^{*} C^{\infty}(G)^{G}$ are actually invariant with respect to the alternative quasi-adjoint action [18] of $G$ on $D$ that can be associated with the 'flipped moment map' $\Lambda^{\prime}:=\Lambda_{R} \Lambda_{L}: D \rightarrow B$. (The elements of $\mathcal{H}(2.32)$ are invariant under both quasi-adjoint actions.) Since $\Lambda=\Lambda_{L} \Lambda_{R}$ can be converted into $\Lambda^{\prime}$ by inversion on the group $D$, it is sufficient to consider only the quasi-adjoint action given by (2.21). 


\section{Reduction of the canonical free systems}

Our goal here is to present the results that permit the identification of a certain symplectic reduction of the canonical free systems $\left(D, \omega_{+}, \mathcal{L}\right)$ and $\left(D, \omega_{+}, \hat{\mathcal{L}}\right)$ of Definition 2.1 with the Ruijsenaars-Schneider system $(P, \omega, L)(1.1)$ and with a natural extension of the dual system $(\hat{P}, \hat{\omega}, \hat{L})(1.3)$, respectively. For this purpose we must take $D=G L(n, \mathbb{C})$, but it is worth stressing that the preliminaries described in Section 2 remain valid in a more general context.

We first review the necessary theoretical background concerning symplectic reduction based on Poisson-Lie symmetry with an equivariant moment map [20]. (The notations $\bar{G}, \bar{B}$, $\Lambda$ used in this overview anticipate the application studied later on.) Consider a symplectic manifold $M$ acted upon smoothly and effectively by a compact Poisson-Lie group $\bar{G}$ in such a way that the action map $\Psi: \bar{G} \times M \rightarrow M$ is Poisson and choose a regular value $\nu \in \bar{B}$ in the image of the moment map ${ }^{2} \Lambda: M \rightarrow \bar{B}$. The $\Lambda$-preimage $F_{\nu}$ of the point $\nu$ is then an embedded submanifold of $M$. The maximal subgroup $\bar{G}_{\nu}<\bar{G}$ which leaves $F_{\nu}$ invariant is called the gauge group. If $\bar{G}_{\nu}$ acts freely on $F_{\nu}$ then, as is well known, there exists a unique manifold structure on the space of orbits, $F_{\nu} / \bar{G}_{\nu}$, such that the canonical projection

$$
\pi: F_{\nu} \rightarrow F_{\nu} / \bar{G}_{\nu}
$$

is a smooth submersion, i.e., $F_{\nu}\left(F_{\nu} / \bar{G}_{\nu}, \bar{G}_{\nu}, \pi\right)$ is a principal fiber bundle. Let us note that at every point $m \in F_{\nu}$ the tangent space $T_{m} F_{\nu}$ has the vertical subspace

$$
V_{m} F_{\nu} \subset T_{m} F_{\nu}
$$

generated by the infinitesimal action of $\bar{G}_{\nu}$. Smooth functions on $F_{\nu} / \bar{G}_{\nu}$ correspond to smooth $\bar{G}_{\nu}$-invariant functions on $F_{\nu}$. The manifold structure on $F_{\nu} / \bar{G}_{\nu}$ is constructed with the aid of local cross sections for the $\bar{G}_{\nu}$ action [7].

In fact, it turns out that $F_{\nu} / \bar{G}_{\nu}$ is a symplectic manifold. It is referred to as the reduced symplectic manifold (or reduced phase space) and the symplectic form $\Omega_{\nu}$ on it is uniquely determined by the requirement

$$
\left.\Omega\right|_{F_{\nu}}=\pi^{*} \Omega_{\nu}
$$

Here $\left.\Omega\right|_{F_{\nu}}$ denotes the pull-back of the original symplectic form $\Omega$ of $M$ on the submanifold $F_{\nu} \subset M$.

The Hamiltonian flow induced by any $\bar{G}$-invariant function $H \in C^{\infty}(M)^{\bar{G}}$ preserves $F_{\nu}$, and the restricted flow is projectable from $F_{\nu}$ to $F_{\nu} / \bar{G}_{\nu}$. The projection gives the flow associated with the reduced Hamiltonian $H^{\nu} \in C^{\infty}\left(F_{\nu} / \bar{G}_{\nu}\right)$ (for which $\left.H\right|_{F_{\nu}}=H^{\nu} \circ \pi$ ) by means of $\Omega_{\nu}$. It further follows that the involutivity of a set of $\bar{G}$-invariant Hamiltonians $\left\{H_{j}\right\} \subset C^{\infty}(M)^{\bar{G}}$ is inherited by the corresponding set of reduced Hamiltonians $\left\{H_{j}^{\nu}\right\} \subset C^{\infty}\left(F_{\nu} / \bar{G}_{\nu}\right)$.

In concrete examples one wishes to exhibit models of the reduced symplectic manifold $\left(F_{\nu} / \bar{G}_{\nu}, \Omega_{\nu}\right)$. In principle, any symplectic manifold that is globally symplectomorphic to $\left(F_{\nu} / \bar{G}_{\nu}, \Omega_{\nu}\right)$ can serve as a model, but the real aim is to construct models as explicitly as

\footnotetext{
${ }^{2}$ The moment map is a Poisson map into the Poisson-Lie group $\bar{B}$ dual to $\bar{G}$. It generates the $\bar{G}$ action via the Poisson bracket on $M$ by $X_{M}[f]=\left\langle X,\{f, \Lambda\}_{M} \Lambda^{-1}\right\rangle$, where $X_{M}$ is the vector field on $M$ corresponding to $X \in \operatorname{Lie}(\bar{G}), f \in C^{\infty}(M)$, and $\langle.,$.$\rangle is the dual pairing.$
} 
possible. The simplest situation occurs when the manifold $F_{\nu}$ is a trivial $\bar{G}_{\nu}$-bundle. Then the reduced symplectic manifold $\left(F_{\nu} / \bar{G}_{\nu}, \Omega_{\nu}\right)$ can be modelled by any global cross section of the $\bar{G}_{\nu}$ action on $F_{\nu}$. To be more precise, we present the following (standard) result.

Lemma 3.1. Suppose that $(\bar{P}, \bar{\omega})$ is a symplectic manifold and $\mathcal{J}: \bar{P} \rightarrow F_{\nu}$ is a smooth injective map such that

1. $\mathcal{J}^{*}\left(\left.\Omega\right|_{F_{\nu}}\right)=\bar{\omega}$,

2. the image $S:=\{\mathcal{J}(y) \mid y \in \bar{P}\}$ intersects every $\bar{G}_{\nu}$-orbit in $F_{\nu}$ exactly in one point.

Then the map $\pi \circ \mathcal{J}: \bar{P} \rightarrow F_{\nu} / \bar{G}_{\nu}$ is a symplectic diffeomorphism, and $(\bar{P}, \bar{\omega})$ can thus serve as a model of the reduced phase space $\left(F_{\nu} / \bar{G}_{\nu}, \Omega_{\nu}\right)$.

Proof. The procedure of symplectic reduction rests on the fact that

$$
\operatorname{Ker}_{m}\left(\left.\Omega\right|_{F_{\nu}}\right)=V_{m} F_{\nu}, \quad \forall m \in F_{\nu}
$$

where $\operatorname{Ker}_{m}\left(\left.\Omega\right|_{F_{\nu}}\right)$ is the annihilator of $\left.\Omega\right|_{F_{\nu}}$ in $T_{m} F_{\nu}$. This equation and $\mathcal{J}^{*}\left(\left.\Omega\right|_{F_{\nu}}\right)=\bar{\omega}$ imply that the tangent (derivative) map $T_{y} \mathcal{J} \equiv(D \mathcal{J})(y): T_{y} \bar{P} \rightarrow T_{\mathcal{J}(y)} F_{\nu}$ is injective and

$$
V_{\mathcal{J}(y)} F_{\nu} \cap T_{y} \mathcal{J}\left(T_{y} \bar{P}\right)=\{0\}, \quad \forall y \in \bar{P} .
$$

Indeed, the hypothesis that, at some $y \in \bar{P}, T_{y} \mathcal{J}(Y) \in V_{\mathcal{J}(y)} F_{\nu}$ for a non-zero $Y \in T_{y} \bar{P}$ would entail that $\mathcal{J}^{*}\left(\left.\Omega\right|_{F_{\nu}}\right)(Y, Z)=0$ for all $Z \in T_{y} \bar{P}$. However, this is excluded by the non-degeneracy of $\mathcal{J}^{*}\left(\left.\Omega\right|_{F_{\nu}}\right)$. The injectivity of $T_{y} \mathcal{J}$ means that the map $\mathcal{J}: \bar{P} \rightarrow F_{\nu}$ is an immersion. Next, being the composition of two smooth maps, $\pi \circ \mathcal{J}: \bar{P} \rightarrow F_{\nu} / \bar{G}_{\nu}$ is smooth and we see from (3.5) that it is also an immersion. Therefore, since $\pi \circ \mathcal{J}$ is injective and surjective by assumption, it must be a submersion, which in particular requires that

$$
T_{\mathcal{J}(y)} F_{\nu}=V_{\mathcal{J}(y)} F_{\nu} \oplus T_{y} \mathcal{J}\left(T_{y} \bar{P}\right), \quad \forall y \in \bar{P} .
$$

It is well known that the one-to-one smooth submersions are precisely the diffeomorphisms. Hence we conclude that the map $\pi \circ \mathcal{J}$ is a diffeomorphism. Finally, using (3.3), we obtain

$$
(\pi \circ \mathcal{J})^{*} \Omega_{\nu}=\mathcal{J}^{*}\left(\pi^{*} \Omega_{\nu}\right)=\mathcal{J}^{*}\left(\left.\Omega\right|_{F_{\nu}}\right)=\bar{\omega} .
$$

Q.E.D.

Remark 3.1. On account of (3.6), the smooth one-to-one map $\Psi \circ\left(\operatorname{id}_{\bar{G}_{\nu}}, \mathcal{J}\right): \bar{G}_{\nu} \times \bar{P} \rightarrow F_{\nu}$ is again a submersion. This implies that the map $\Psi \circ\left(\operatorname{id}_{\bar{G}_{\nu}}, \mathcal{J}\right)$ is a diffeomorphism. Hence the map $\mathcal{J}$ is an embedding and the restriction of the projection $\pi$ to $S=\mathcal{J}(\bar{P})$ yields a diffeomorphism, $\pi_{S}: S \rightarrow F_{\nu} / \bar{G}_{\nu}$. Moreover, if we define $\sigma: F_{\nu} / \bar{G}_{\nu} \rightarrow F_{\nu}$ by $\sigma(b):=\mathcal{J}(y)$ for the unique $y \in \bar{P}$ such that $b=\pi(\mathcal{J}(y))$, then $\sigma$ is a smooth global section of the bundle $\pi: F_{\nu} \rightarrow F_{\nu} / \bar{G}_{\nu}$ in the usual sense, i.e., $\sigma \in C^{\infty}\left(F_{\nu} / \bar{G}_{\nu}, F_{\nu}\right)$ and $\pi \circ \sigma=\operatorname{id}_{F_{\nu} / \bar{G}_{\nu}}$. This follows by noting that $\sigma=\iota_{S} \circ \pi_{S}^{-1}$, where $\iota_{S}: S \rightarrow F_{\nu}$ is the tautological injection.

Remark 3.2. We call the map $\mathcal{J}$ of Lemma 3.1 a global cross section of the $\bar{G}_{\nu}$ action on $F_{\nu}$. The same term can be used to refer to the image $S$ of $\mathcal{J}$, too, but here we adopt the more 
widespread terminology that refers to $S$ as a global gauge slice. Note that $\left(S,\left.\Omega\right|_{S}\right)$ can be also thought of as a model of the reduced phase space, since it is symplectomorphic to $(\bar{P}, \bar{\omega})$ by $\mathcal{J}$.

From now on we take the unreduced symplectic manifold to be the Heisenberg double,

$$
(M, \Omega):=\left(G L(n, \mathbb{C}), \omega_{+}\right)
$$

and identify the effectively acting symmetry group $\bar{G}$ with $U(n)$ divided by its center. As explained in Remark 2.1, $\bar{G}$ acts by the quasi-adjoint action (2.21) according to

$$
\Psi([g], K):=g \triangleright K, \quad \forall[g] \in \bar{G}, \forall K \in G L(n, \mathbb{C}),
$$

where $g \in U(n)$ is an arbitrary representative of $[g] \in U(n) / U(1)$. In Definition 2.1 of Section 2, we introduced two families of $\bar{G}$ Poisson-Lie symmetric Hamiltonian systems, namely $\left(G L(n, \mathbb{C}), \omega_{+}, \mathcal{L}\right)$ and $\left(G L(n, \mathbb{C}), \omega_{+}, \hat{\mathcal{L}}\right)$. Since these canonical free systems have the same symplectic structure $\omega_{+}$and they are Poisson-Lie symmetric with respect to the same action of $\bar{G}$, we can consider their simultaneous symplectic reduction based on a moment map value $\nu$. In our letter [11], we identified the (parameter-dependent) value $\nu(x) \in \bar{B}$ such that the symplectic reduction of the system $\left(G L(n, \mathbb{C}), \omega_{+}, \mathcal{L}\right)$ gives the original Ruijsenaars-Schneider system $(P, \omega, L)$ (1.1). An enhanced formulation of this result is given by Theorems 3.1 and 3.2 below. Later on, we shall formulate Theorems 3.3 and 3.4, which claim that the symplectic reduction of $\left(G L(n, \mathbb{C}), \omega_{+}, \hat{\mathcal{L}}\right)$ with respect to the same $\nu(x)$ gives an extension of the dual Ruijsenaars-Schneider system $(\hat{P}, \hat{\omega}, \hat{L})(1.3)$.

In order to formulate precisely the above mentioned theorems, first we have to recall some notations and results from our previous paper [11. Thus let $x$ be a real non-zero parameter and denote by $\nu(x)$ the element of the group $\bar{B} \simeq S B<B$ (remember (2.28) ) such that

$$
\nu(x)_{k k}=1, \quad \forall k, \quad \nu(x)_{k l}=\left(1-e^{-x}\right) e^{\frac{(l-k) x}{2}}, \quad \forall k<l .
$$

There holds the equation

$$
\nu(x) \nu(x)^{\dagger}=e^{-x}\left[\mathbf{1}_{n}+\frac{e^{n x}-1}{n} v(x) v(x)^{\dagger}\right]
$$

where the components of the real column vector $v(x)$ read

$$
v_{k}(x)=\sqrt{\frac{n\left(e^{x}-1\right)}{1-e^{-n x}}} e^{-\frac{k x}{2}}, \quad \forall k=1, \ldots, n .
$$

The isotropy subgroup $G_{\nu(x)}<U(n)$ is the direct product $G_{v(x)} \times U(1)$, where $G_{v(x)}<U(n)$ is the isotropy group of $v(x) \in \mathbb{C}^{n}$ and $U(1)$ is the center of $U(n)$. The corresponding subgroup $\bar{G}_{\nu(x)}$ of the effective symmetry group $\bar{G}=U(n) / U(1)$ admits the natural isomorphisms

$$
\bar{G}_{\nu(x)} \simeq G_{\nu(x)} / U(1) \simeq G_{v(x)},
$$

and therefore we can identify $\bar{G}_{\nu(x)}$ with $G_{v(x)}$. 
Let $\Lambda: G L(n, \mathbb{C}) \rightarrow \bar{B} \simeq S B(2.28)$ be the moment map of the quasi-adjoint action (3.9) of $\bar{G}=U(n) / U(1)$. With $\nu(x)$ in (3.10), consider the symplectic reduction of the Heisenberg double $\left(G L(n, \mathbb{C}), \omega_{+}\right)$defined by imposing the constraint

$$
\Lambda(K):=\Lambda_{L}(K) \Lambda_{R}(K)=\nu(x), \quad K \in G L(n, \mathbb{C}) .
$$

The associated gauge group is given by $\bar{G}_{\nu(x)}(\underline{3.13})$, and we have the following basic lemma.

Lemma 3.2 ([11]). The set $F_{\nu(x)}$ consisting of the solutions of the moment map constraint (3.14) is an embedded submanifold of $G L(n, \mathbb{C})$ and the compact group $\bar{G}_{\nu(x)}$ acts freely on it.

Lemma 3.2 was proven in [11] by finding explicitly all solutions of the moment map constraint (3.14). For completeness, in Appendix A we offer an alternative proof of the fact that $F_{\nu(x)}$ is an embedded submanifold by showing that the element $\nu(x)$ is a regular value of the moment map $\Lambda$. It follows from the lemma that the orbit space $F_{\nu(x)} / \bar{G}_{\nu(x)}$ is a smooth manifold: the base of the principal fiber bundle with total space $F_{\nu(x)}$ and structure group $\bar{G}_{\nu(x)}$. For any $H \in \mathcal{H}(2.29)$ (respectively $\hat{H} \in \hat{\mathcal{H}}$ ), the reduction of the Hamiltonian system $\left(G L(n, \mathbb{C}), \omega_{+}, H\right)$ yields a system with complete Hamiltonian flow on the reduced phase space, which can be constructed by projecting the original flow given by Propositions 2.1 (respectively Proposition 2.2). Before giving a characterization of the reduction of the canonical free system $\left(G L(n, \mathbb{C}), \omega_{+}, \mathcal{L}\right)$ of Definition 2.1, next we present a convenient description of the phase space $(P, \omega)$ of the original Ruijsenaars-Schneider system (1.1).

Let $\mathbb{T}_{n}^{0}$ denote the regular part of the standard maximal torus $\mathbb{T}_{n}<G=U(n)$. The symmetric group $S(n)$ acts freely on $\mathbb{T}_{n}^{0}$, any $\sigma \in S(n)$ acts by permuting the diagonal entries of the elements $T=\operatorname{diag}\left(T_{1}, \ldots, T_{n}\right) \in \mathbb{T}_{n}^{0}$. Thus the corresponding space of $S(n)$-orbits

$$
Q(n):=\mathbb{T}_{n}^{0} / S(n)
$$

is a smooth manifold. This is the set of unordered $n$-tuples of pairwise distinct points of the circle $S^{1}=U(1)$. The phase space of the trigonometric Ruijsenaars-Schneider system, when interpreted as a many-body system of indistinguishable particles, is in fact given by

$$
(P, \omega):=\left(T^{*} Q(n), \Omega_{T^{*} Q(n)}\right),
$$

where $\Omega_{T^{*} Q(n)}$ is the canonical symplectic form of the cotangent bundle $T^{*} Q(n)$. Note that $S(n)$ acts freely on $T^{*} \mathbb{T}_{n}^{0}$, too, by the cotangent lift of the $S(n)$-action on $\mathbb{T}_{n}^{0}$. If we use the realization

$$
T^{*} \mathbb{T}_{n}^{0} \simeq \mathbb{T}_{n}^{0} \times \mathbb{R}^{n}=\left\{\left(e^{2 \mathrm{i} q}, p\right)\right\}
$$

then this $S(n)$-action operates by the simultaneous permutations of the entries of $e^{2 \mathrm{iq} q}$ and $p$. We identify $p$ with the diagonal matrix $p \equiv \operatorname{diag}\left(p_{1}, \ldots, p_{n}\right)$ and choose the normalization of the natural symplectic form $\Omega_{T^{*} \mathbb{T}_{n}^{0}}$ as

$$
\Omega_{T^{*} \mathbb{T}_{n}^{0}} \equiv \sum_{k=1}^{n} d p_{k} \wedge d q_{k}=\frac{1}{2} \Im \operatorname{tr}\left(d p \wedge e^{-2 \mathrm{i} q} d e^{2 \mathrm{i} q}\right) .
$$

The point is that $\mathbb{T}_{n}^{0}$ is a covering space of $Q(n)$, and $T^{*} \mathbb{T}_{n}^{0}$ is a symplectic covering space of $T^{*} Q(n)$. To state this formally, we have

$$
\pi_{1}^{*}\left(\Omega_{T^{*} Q(n)}\right)=\Omega_{T^{*} \mathbb{T}_{n}^{0}},
$$


where

$$
\pi_{1}: T^{*} \mathbb{T}_{n}^{0} \rightarrow\left(T^{*} \mathbb{T}_{n}^{0}\right) / S(n) \equiv T^{*}\left(\mathbb{T}_{n}^{0} / S(n)\right) \equiv T^{*} Q(n)
$$

is the natural submersion. Therefore one can identify the Poisson algebra of the smooth functions on $T^{*} Q(n)$ with the Poisson algebra of the smooth $S(n)$-invariant functions on $T^{*} \mathbb{T}_{n}^{0}$. For example, one may regard the Lax matrix $L(q, p)$ (1.1) as a function on $T^{*} \mathbb{T}_{n}^{0}$, with its spectral invariants (symmetric functions) giving the commuting Hamiltonians on $T^{*} Q(n)$. Actually $Q(n)$ is a rather non-trivial manifold, and the ensuing technical complications are avoided if one works with the $S(n)$-invariant functions on $T^{*} \mathbb{T}_{n}^{0}$.

Theorem 3.1. Consider the smooth map $\tilde{\mathcal{I}}: T^{*} \mathbb{T}_{n}^{0} \rightarrow G L(n, \mathbb{C})$ defined by the formula

$$
\begin{gathered}
\tilde{\mathcal{I}}\left(e^{2 \mathrm{i} q}, p\right)_{k k}=e^{-\frac{p_{k}}{2}-2 \mathrm{i} q_{k}} \prod_{m<k}\left[1+\frac{\sinh ^{2} \frac{x}{2}}{\sin ^{2}\left(q_{k}-q_{m}\right)}\right]^{-\frac{1}{4}} \prod_{m>k}\left[1+\frac{\sinh ^{2} \frac{x}{2}}{\sin ^{2}\left(q_{k}-q_{m}\right)}\right]^{\frac{1}{4}}, \\
\tilde{\mathcal{I}}\left(e^{2 \mathrm{i} q}, p\right)_{k l}=0, \quad k>l, \quad \tilde{\mathcal{I}}\left(e^{2 \mathrm{i} q}, p\right)_{k l}=\tilde{\mathcal{I}}\left(e^{2 \mathrm{i} q}, p\right)_{l l} \prod_{m=1}^{l-k} \frac{e^{\frac{x}{2}} e^{2 \mathrm{i} q_{l}}-e^{-\frac{x}{2}} e^{2 \mathrm{i} q_{k+m}}}{e^{2 \mathrm{i} q_{l}}-e^{2 \mathrm{i} q_{k+m-1}}}, \quad k<l,
\end{gathered}
$$

where $T^{*} \mathbb{T}_{n}^{0}$ is parametrized according to (3.17). This map is injective, its image lies in $F_{\nu(x)}$, and it enjoys the property

$$
\tilde{\mathcal{I}}^{*}\left(\omega_{+}\right)=\Omega_{T^{*} \mathbb{T}_{n}^{0}}
$$

Moreover, $\tilde{\mathcal{I}}$ descends to a diffeomorphism $\mathcal{I}: T^{*} Q(n) \rightarrow F_{\nu(x)} / \bar{G}_{\nu(x)}$ defined by the equality

$$
\mathcal{I} \circ \pi_{1}=\pi \circ \tilde{\mathcal{I}}
$$

with the projections $\pi_{1}: T^{*} \mathbb{T}_{n}^{0} \rightarrow T^{*} Q(n), \pi: F_{\nu(x)} \rightarrow F_{\nu(x)} / \bar{G}_{\nu(x)}$, and there holds the relation

$$
\mathcal{I}^{*}\left(\Omega_{\nu(x)}\right)=\Omega_{T^{*} Q(n)} .
$$

Thus $(P, \omega)$ (3.16) is a model of the reduced phase space $\left(F_{\nu(x)} / \bar{G}_{\nu(x)}, \Omega_{\nu(x)}\right)$.

Remark 3.3. The image of $T^{*} \mathbb{T}_{n}^{0}$ by $\tilde{\mathcal{I}}$ is a symplectic submanifold $\tilde{S} \subset G L(n, \mathbb{C}) .\left(\tilde{S},\left.\omega_{+}\right|_{\tilde{S}}\right)$ and $T^{*} \mathbb{T}_{n}^{0}$ are symplectomorphic by $\tilde{\mathcal{I}}$, and furnish symplectic covering spaces of the reduced phase space.

Theorem 3.2. The composition map $\mathcal{L} \circ \tilde{\mathcal{I}}$, with $\mathcal{L}$ in (2.60), gives (up to an inessential similarity transformation) the Lax matrix $L$ (1.1) of the original Ruijsenaars-Schneider system, where $L$ is regarded as a function on the covering space $T^{*} \mathbb{T}_{n}^{0}$ of $P=T^{*} Q(n)$.

The previous Theorems 3.1 and 3.2 were essentially obtained in our letter [11, but there the covering space aspects and some other non-trivial details were omitted. We shall give full proofs of them later in this paper. The forthcoming Theorems 3.3 and 3.4 encapsulate the main original results obtained in this paper. They are technically more involved than Theorems 3.1 and 3.2 and, in order to formulate them, we first need to define several matrix-valued functions of various sets of arguments. The reader who does not wish to be slowed down in following the conceptual line of presentation may skip the details of these definitions on first reading and continue directly to the statements of the theorems afterwards. 
Definition 3.1. For any $x \in \mathbb{R}$, define the following subsets of $\mathbb{R}^{n}$ :

$$
\mathfrak{C}_{x}:=\left\{\hat{p} \in \mathbb{R}^{n} \mid \hat{p}_{l}-\hat{p}_{l+1}>\frac{|x|}{2}, 1 \leq l<n\right\}, \quad \overline{\mathfrak{C}}_{x}:=\left\{\hat{p} \in \mathbb{R}^{n} \mid \hat{p}_{l}-\hat{p}_{l+1} \geq \frac{|x|}{2}, 1 \leq l<n\right\}
$$

Remark 3.4. We shall refer to the elements of $\mathfrak{C}_{x}$ as the interior elements of $\overline{\mathfrak{C}}_{x}$. Note that $\overline{\mathfrak{C}}_{0}$ is the standard Weyl chamber associated with $g l(n, \mathbb{C})$ and the phase space of the system $(\hat{P}, \hat{\omega}, \hat{L})$ is $\hat{P}=\mathbb{T}_{n} \times \mathfrak{C}_{x}$. We often identify an element of $\mathbb{R}^{n}$ with a corresponding $n \times n$ diagonal matrix. For example, $\hat{q}$ in (1.4) parametrizes $\mathbb{T}_{n}$ by $e^{\mathrm{i} \hat{q}}$ and we may use $\hat{p} \simeq \operatorname{diag}\left(\hat{p}_{1}, \ldots, \hat{p}_{n}\right)$.

Definition 3.2. Consider the phase space $\hat{P}=\mathbb{T}_{n} \times \mathfrak{C}_{x}(1.4)$ of the dual Ruijsenaars-Schneider system and the symplectic manifold $\hat{P}_{c}:=\mathbb{C}^{n-1} \times \mathbb{C}^{\times}$, where $\mathbb{C}^{\times}$denotes the complex plane without the origin and the symplectic form $\hat{\omega}_{c}$ on $\hat{P}_{c}$ is defined by

$$
\hat{\omega}_{c}:=\frac{\mathrm{i} d Z \wedge d \bar{Z}}{2 \bar{Z} Z}+\operatorname{sign}(x) \sum_{j=1}^{n-1} \mathrm{i} d z_{j} \wedge d \bar{z}_{j}, \quad Z \in \mathbb{C}^{\times}, \quad z \in \mathbb{C}^{n-1} .
$$

Define the smooth injective map $\mathcal{Z}_{x}: \hat{P} \rightarrow \hat{P}_{c}$ by

$$
\begin{aligned}
& z_{j}(x, \hat{q}, \hat{p})=\left(\hat{p}_{j}-\hat{p}_{j+1}-x / 2\right)^{\frac{1}{2}} \prod_{k=j+1}^{n} e^{-\mathrm{i} \hat{q}_{k}}, j=1, \ldots, n-1, Z(x, \hat{q}, \hat{p})=e^{-\hat{p}_{1}} \prod_{k=1}^{n} e^{-\mathrm{i} \hat{q}_{k}}, x>0 \\
& z_{j}(x, \hat{q}, \hat{p})=\left(\hat{p}_{j}-\hat{p}_{j+1}+x / 2\right)^{\frac{1}{2}} \prod_{k=1}^{j} e^{-\mathrm{i} \hat{q}_{k}}, j=1, \ldots, n-1, Z(x, \hat{q}, \hat{p})=e^{-\hat{p}_{n}} \prod_{k=1}^{n} e^{-\mathrm{i} \hat{q}_{k}}, x<0 .
\end{aligned}
$$

Remark 3.5. One can check that $\mathcal{Z}_{x}$ is a symplectic embedding of $(\hat{P}, \hat{\omega})$ into $\left(\hat{P}_{c}, \hat{\omega}_{c}\right)$, i.e.,

$$
\mathcal{Z}_{x}^{*} \hat{\omega}_{c}=\hat{\omega}
$$

with $\hat{\omega}$ in (1.4). The $\mathcal{Z}_{x}$-image of $\hat{P}$ in $\hat{P}_{c}$ is a dense open submanifold; $\hat{P}_{c} \backslash \mathcal{Z}_{x}(\hat{P})$ consists of the points for which one or more of the complex coordinates $z_{j}$ is equal to zero. It is important to note that the same embedding of $\hat{P}$ into $\hat{P}_{c}$ was also used in [30] $]^{3}$. This fact, together with the requirements forced on us by the technical analysis in Section 5, motivated Definition 3.2.

Definition 3.3. By introducing the 'special index' $a:=n$ for $x>0$ and $a:=1$ for $x<0$, define the $n \times n$ orthogonal matrix $\kappa_{L}(x)$ by

$$
\kappa_{L}(x)_{a a}=\frac{v_{a}(x)}{\sqrt{n}}, \kappa_{L}(x)_{i j}=\delta_{i j}-\frac{v_{i}(x) v_{j}(x)}{n+\sqrt{n} v_{a}(x)}, \kappa_{L}(x)_{i a}=-\kappa_{L}(x)_{a i}=\frac{v_{i}(x)}{\sqrt{n}}, i, j \neq a,
$$

where $v(x)$ is given by (3.12). Then consider $z \in \mathbb{C}^{n-1}$ and introduce the smooth functions

$$
Q_{j k}(x, z)=\sqrt{\frac{\sinh \left(\sum_{l=j}^{k-1} z_{l} \bar{z}_{l}+(k-j) \frac{|x|}{2}-\frac{x}{2}\right)}{\sinh \left(\sum_{l=j}^{k-1} z_{l} \bar{z}_{l}+(k-j) \frac{|x|}{2}\right)}}, \quad 1 \leq j<k \leq n,
$$

\footnotetext{
${ }^{3}$ One can see this from Eq. (1.73) in [30], where the completion of the dual phase space was formulated in terms of a covering space of $\hat{P}$.
} 
with $Q_{j k}(x, z):=Q_{k j}(-x, z)$ for $j>k$. By using the above notations and $J(y):=\sqrt{\frac{\sinh y}{y}}$ for $y \neq 0, J(0):=1$, define the smooth $n \times n$ matrix function $\hat{\zeta}(x, z)$ as

$$
\begin{aligned}
& \hat{\zeta}(x, z)_{a a}=\sqrt{\frac{\sinh \frac{x}{2}}{\sinh \frac{n x}{2}}} \prod_{l \neq a} Q_{a l}(x, z), \quad \hat{\zeta}(x, z)_{a j}=-\overline{\hat{\zeta}(x, z)_{j a}}, \quad j \neq a, \\
& \hat{\zeta}(x, z)_{j n}=\sqrt{\frac{\sinh \frac{x}{2}}{\sinh \frac{n x}{2}}} \frac{z_{j} J\left(z_{j} \bar{z}_{j}\right)}{\sqrt{\sinh \left(z_{j} \bar{z}_{j}+\frac{x}{2}\right)}} \prod_{l \neq j, j+1} Q_{j l}(x, z), \quad x>0, \quad j \neq n, \\
& \hat{\zeta}(x, z)_{j 1}=\sqrt{\frac{\sinh \frac{x}{2}}{\sinh \frac{n x}{2}}} \frac{z_{j-1} J\left(z_{j-1} \bar{z}_{j-1}\right)}{\sqrt{\sinh \left(z_{j-1} \bar{z}_{j-1}-\frac{x}{2}\right)}} \prod_{l \neq j-1, j} Q_{j l}(x, z), \quad x<0, \quad j \neq 1, \\
& \hat{\zeta}(x, z)_{j k}=\delta_{j k}+\frac{\hat{\zeta}(x, z)_{j a} \hat{\zeta}(x, z)_{a k}}{1+\hat{\zeta}(x, z)_{a a}}, \quad j, k \neq a .
\end{aligned}
$$

Next, define the smooth $n \times n$ matrix function $\hat{\theta}(x, z)$ for $x>0$ as

$$
\begin{gathered}
\hat{\theta}(x, z)_{j k}=\frac{\left(\sinh \frac{n x}{2}\right) \operatorname{sign}(k-j-1) \hat{\zeta}(x, z)_{j n} \hat{\zeta}(-x, z)_{1 k}}{\sinh \left(\sum_{l=\min (k, j)}^{\max (k, j)-1} z_{l} \bar{z}_{l}+|k-j-1| \frac{x}{2}\right)}, \quad k \neq j+1, \\
\hat{\theta}(x, z)_{j, j+1}=\frac{-\sinh \frac{x}{2}}{\sinh \left(z_{j} \bar{z}_{j}+\frac{x}{2}\right)} \prod_{l \neq j, j+1} Q_{j l}(x, z) Q_{j+1, l}(-x, z),
\end{gathered}
$$

and for $x<0$ as

$$
\hat{\theta}(x, z)=\hat{\theta}(-x, z)^{\dagger} .
$$

Finally, let $\Delta(x, z, Z)$ be the diagonal matrix function on $\hat{P}_{c}$ given for $x>0$ by the components

$$
\Delta_{1}(x, z, Z)=Z, \quad \Delta_{j}(x, z, Z)=|Z| \exp \left(\sum_{l=1}^{j-1} z_{l} \bar{z}_{l}+(j-1) \frac{x}{2}\right), \quad j=2, \ldots, n,
$$

and for $x<0$ by the components

$$
\Delta_{n}(x, z, Z)=Z, \quad \Delta_{j}(x, z, Z)=|Z| \exp \left(-\sum_{l=j}^{n-1} z_{l} \bar{z}_{l}+(n-j) \frac{x}{2}\right), \quad j=1, \ldots, n-1 .
$$

Remark 3.6. The origin of the above formulae will become clear in Section 5, and there we shall demonstrate the unitarity of the matrices $\hat{\theta}(x, z)$ and $\hat{\zeta}(x, z)$ for all values of their arguments.

Recall Remark 3.2 concerning our terminology for a global cross section.

Theorem 3.3. The symplectic manifold $\left(\hat{P}_{c}, \hat{\omega}_{c}\right)$ (3.27) is a model of the reduced phase space $\left(F_{\nu(x)} / \bar{G}_{\nu(x)}, \Omega_{\nu(x)}\right)$. With the notations introduced in Definition 3.3, the map $\hat{\mathcal{I}}: \hat{P}_{c} \rightarrow F_{\nu(x)}$ given by

$$
\hat{\mathcal{I}}(z, Z):=\left(\kappa_{L}(x) \hat{\zeta}(x, z)^{-1}\right) \triangleright\left(\Delta(x, z, Z) \hat{\theta}(x, z)^{-1}\right)
$$


is a global cross section, and $\pi \circ \hat{\mathcal{I}}: \hat{P}_{c} \rightarrow F_{\nu(x)} / \bar{G}_{\nu(x)}$ is a symplectomorphism.

Theorem 3.4. By using the symplectic embedding $\mathcal{Z}_{x}: \hat{P} \rightarrow \hat{P}_{c}$ introduced in Definition 3.2 and $\hat{\mathcal{L}}$ defined in (2.60), the composition map $\hat{\mathcal{L}} \circ \hat{\mathcal{I}} \circ \mathcal{Z}_{x}$ gives (up to an inessential similarity transformation) the Lax matrix $\hat{L}$ (1.3) of the dual Ruijsenaars-Schneider system $(\hat{P}, \hat{\omega}, \hat{L})$.

To sum up, Theorems 3.1 and 3.2 state that the original trigonometric Ruijsenaars-Schneider system $(P, \omega, L)$ is exactly the result of the symplectic reduction of the canonical free system $\left(G L(n, \mathbb{C}), \omega_{+}, \mathcal{L}\right)$. Theorems 3.3 and 3.4 affirm that the reduction of the other canonical free system $\left(G L(n, \mathbb{C}), \omega_{+}, \hat{\mathcal{L}}\right)$ gives a certain integrable system $\left(\hat{P}_{c}, \hat{\omega}_{c}, \hat{\mathcal{L}} \circ \hat{\mathcal{I}}\right)$ which can be viewed (due to the non-surjectivity of the map $\mathcal{Z}_{x}: \hat{P} \rightarrow \hat{P}_{c}$ ) as an extension of the dual RuijsenaarsSchneider system $(\hat{P}, \hat{\omega}, \hat{L})$. Moreover, together with Remark 3.5, they ensure that the extended dual Ruijsenaars-Schneider system $\left(\hat{P}_{c}, \hat{\omega}_{c}, \hat{\mathcal{L}} \circ \hat{\mathcal{I}}\right)$ coincides with the 'minimal completion' of the system $(\hat{P}, \hat{\omega}, \hat{L})$ constructed by Ruijsenaars by means of the direct method [30]. The guiding principle behind his extension of $\hat{P}$ was the aim to obtain a bijective correspondence between the phase spaces of the dual pair of systems. At the same time, the extension gave rise to the completion of the dual flows, which are not complete on $\hat{P}$. It is pleasing that Ruijsenaars' minimal completion comes about naturally from the symplectic reduction. In this framework the geometric origin of the duality symplectomorphism between $(P, \omega)$ and $\left(\hat{P}_{c}, \hat{\omega}_{c}\right)$, which has been established in [30] by a complicated web of arguments, becomes transparent: any two models of the reduced phase space are naturally symplectomorphic to each other. The natural symplectomorphism maps to each other those points of the two different models that correspond to the same point of the reduced phase space.

We shall further discuss in Section 6 why the geometrically induced symplectomorphism between $(P, \omega)$ and $\left(\hat{P}_{c}, \hat{\omega}_{c}\right)$ is the same as the duality map (alias 'action-angle map') constructed in $[30]$.

\section{$4 \quad$ Proofs of Theorems 3.1 and 3.2}

Many ingredients of the proofs that follow were already given in our previous paper [11] but here we present all this material in a more complete and natural way. In particular, we shall explain how the non-trivial topology of the configuration space of indistinguishable particles on the circle (see also Appendix B) is reflected in the symplectic reduction and why this aspect of the story explains the geometric origin of the important formula (3.13) of [11]. We recall that the somewhat complicated formula (3.13) of [1] (which appears as (4.19) below) relates the group theoretically simplest coordinates on the reduced phase space with the cotangent-bundle coordinates in which the Ruijsenaars-Schneider Hamiltonians are usually expressed.

Let $A<B$ denote the subgroup of diagonal matrices with positive real entries and $N<B$ the subgroup of upper-triangular matrices with unit diagonal. Define the smooth function $\mathcal{N}: \mathbb{T}_{n}^{0} \rightarrow N$ by the formula

$$
\mathcal{N}(T)_{k l}=\prod_{m=1}^{l-k} \frac{e^{\frac{x}{2}} T_{l}-e^{-\frac{x}{2}} T_{k+m}}{T_{l}-T_{k+m-1}}, \quad \forall k<l,
$$


and introduce the subset $\tilde{S} \subset G L(n, \mathbb{C})$ as follows:

$$
\tilde{S}:=\left\{\mathcal{N}(T) a T^{-1} \mid a \in A, T \in \mathbb{T}_{n}^{0}\right\} .
$$

Lemma 4.1. The set $\tilde{S}$ lies in the constraint-manifold $F_{\nu(x)} \subset G L(n, \mathbb{C})$ and it intersects every orbit of the gauge group $\bar{G}_{\nu(x)}$ acting on $F_{\nu(x)}$. Every $K \in F_{\nu(x)}$ with $\Xi_{R}(K) \in \mathbb{T}_{n}$ belongs to $\tilde{S}$. The map

$$
\mathbb{T}_{n}^{0} \times A \rightarrow F_{\nu(x)}, \quad(T, a) \mapsto \mathcal{N}(T) a T^{-1}
$$

is an embedding, and the corresponding pull-back of the form $\omega_{+}$is the symplectic form

$$
\omega_{\tilde{S}}=\Im \operatorname{tr}\left(T^{-1} d T \wedge a^{-1} d a\right) .
$$

Proof. The statement is just a reformulation of part of Theorem 1 of [11. The fact that $\tilde{S}$ is an embedded submanifold of $G L(n, \mathbb{C})$ is obvious from the Iwasawa decomposition, which also implies by Lemma 3.2 that $\tilde{S}$ is an embedded submanifold of $F_{\nu(x)}$. Q.E.D.

Lemma 4.2. For every fixed $K \in \tilde{S}$ and permutation $\sigma \in S(n)$ there exists a unique element $[g(K, \sigma)] \in \bar{G}_{\nu(x)}$ for which

$$
\Psi([g(K, \sigma)], K) \in \tilde{S} \quad \text { and } \quad \Xi_{R}(\Psi([g(K, \sigma)], K))=\sigma\left(\Xi_{R}(K)\right),
$$

where $\Psi$ denotes the action (3.9) and $\sigma(T)$ is obtained by permuting the entries of any $T \in \mathbb{T}_{n}^{0}$. All gauge transformations that map $K \in \tilde{S}$ to $\tilde{S}$ are of the above type, and the formula

$$
\Phi: S(n) \times \tilde{S} \rightarrow \tilde{S}, \quad \Phi_{\sigma}(K):=\Phi(\sigma, K):=\Psi([g(K, \sigma)], K)
$$

defines a smooth, free action of $S(n)$ on $\tilde{S}$, which preserves the symplectic form $\omega_{\tilde{S}}$.

Proof. This can be extracted from [11], too, and thus we can be brief here. First, for fixed $K \in \tilde{S}$ and $\sigma \in S(n)$ there cannot exist two gauge transformations subject to (4.5), since the action of $\bar{G}_{\nu(x)}$ is free on $F_{\nu(x)}$ and two different elements $K_{1}, K_{2} \in \tilde{S}$ satisfying $\Xi_{R}\left(K_{1}\right)=$ $\Xi_{R}\left(K_{2}\right)$ are never gauge equivalent [11]. Second, because of the second relation in (2.35) and the surjectivity of the map $g_{K}: U(n) \rightarrow U(n)$ given by $g_{K}(\eta)=\Xi_{R}\left(\eta \Lambda_{L}(K)\right)$ for each $K$, it is clear that for any $K \in \tilde{S}$ and $\sigma \in S(n)$ there exists some $\eta \in G$ for which

$$
\Xi_{R}(\eta \triangleright K)=\sigma\left(\Xi_{R}(K)\right) .
$$

This implies that

$$
\left.\operatorname{Dress}_{\eta}(\nu(x))\right)_{j j}=\Lambda(\eta \triangleright K)_{j j}=1, \quad \forall j,
$$

where we used both the equivariance of the moment map as well the formula (2.22), which shows that $\Lambda(K)_{j j}=1$ if $K=b T^{-1}$ for some $b \in B$ and $T \in \mathbb{T}_{n}$. Next, it is not difficult to see (e.g. from the proof of Lemma 1 in [11]) that for any element $\operatorname{Dress}_{\eta}(\nu(x))$ with unit diagonal there exists some $\tau \in \mathbb{T}_{n}$ for which $\operatorname{Dress}_{\eta}(\nu(x))=\operatorname{Dress}_{\tau}(\nu(x))$. Then it follows from (4.7) that $\Xi_{R}\left(\tau^{-1} \eta \triangleright K\right)=\sigma\left(\Xi_{R}(K)\right) \in \mathbb{T}_{n}$. Consequently, $[g(K, \sigma)]:=\left[\tau^{-1} \eta\right] \in \bar{G}_{\nu(x)}$ is the required element. 
Each gauge transformation that maps $K \in \tilde{S}$ to $\tilde{S}$ is associated with some $\sigma \in S(n)$ according to (4.5), since these gauge transformations act by some permutation on $\Xi_{R}(K)$ (again because of (2.35) ). It is clear from the established uniqueness property that (4.6) defines indeed a smooth, free action of $S(n)$ on $\tilde{S}$. This action preserves $\omega_{\tilde{S}}$ since it is given by gauge transformations (the gauge transformations preserve $\left.\omega_{+}\right|_{F_{\nu}(x)}$, and $\omega_{\tilde{S}}$ is the pull-back of $\left.\omega_{+}\right|_{F_{\nu}(x)}$ on $\tilde{S})$. Q.E.D.

The following important formula was found by first making a detailed inspection in the $n=2$ case, and then generalizing the result for arbitrary $n$.

Lemma 4.3. The action of the transposition $\sigma_{k, k+1} \in S(n)$ on $\mathcal{N}(T) a T^{-1} \in \tilde{S}$ is given explicitly by the formula

$$
\Phi_{\sigma_{k, k+1}}\left(\mathcal{N}(T) a T^{-1}\right)=\mathcal{N}\left(\sigma_{k, k+1}(T)\right) \hat{a} \sigma_{k, k+1}(T)^{-1},
$$

where $\hat{a}_{j}=a_{j}$ if $j \notin\{k, k+1\}$ and

$$
\hat{a}_{k}=a_{k+1} W_{k}(T), \quad \hat{a}_{k+1}=\frac{a_{k}}{W_{k}(T)} \text { with } W_{k}(T):=\left[1+\frac{\sinh ^{2} \frac{x}{2}}{\sin ^{2}\left(q_{k}-q_{k+1}\right)}\right]^{\frac{1}{2}}, T=e^{2 \mathrm{i} q} .
$$

Proof. Fix $1 \leq k \leq(n-1)$ and $K=\mathcal{N}(T) a T^{-1} \in \tilde{S}$. For any $\gamma \in(0, \pi)$ define the matrix $g(\gamma) \in S U(2)$ by

$$
g(\gamma):=\left[\begin{array}{ll}
\alpha & \beta \\
\beta & \bar{\alpha}
\end{array}\right], \quad \alpha:=\left(\cos \gamma+\mathrm{i} \sin \gamma \tanh \frac{x}{2}\right), \quad \beta:=\mathrm{i} \sin \gamma / \cosh \frac{x}{2} .
$$

Then introduce the element $g_{k}(\gamma) \in U(n)$ by

$$
g_{k}(\gamma):=\operatorname{diag}(\Gamma, \ldots, \Gamma, g(\gamma), \Gamma, \ldots, \Gamma), \quad \Gamma:=e^{\mathrm{i} \gamma},
$$

where the first string of $\Gamma$ 's occupies the first $(k-1)$-entries along the diagonal. Introduce similarly the matrix $\chi_{k}(\gamma) \in U(n)$ by

$$
\chi_{k}(\gamma):=\operatorname{diag}(\Gamma, \ldots, \Gamma, \chi, \Gamma, \ldots, \Gamma) \quad \text { with } \quad \chi:=\left[\begin{array}{cc}
0 & \mathrm{i} \\
\mathrm{i} & 0
\end{array}\right]
$$

It is readily verified that the vector $v(x)$ (3.12) is an eigenvector of $g_{k}(\gamma)$,

$$
g_{k}(\gamma) v(x)=e^{\mathrm{i} \gamma} v(x)
$$

which implies that $g_{k}(\gamma) \triangleright \nu(x)=\nu(x)$, i.e., $\left[g_{k}(\gamma)\right]$ belongs to the gauge group $\bar{G}_{\nu(x)}$. It is also straightforward to check that if $\gamma$ is determined by the equality

$$
\cot \gamma=(\tanh x / 2) \cot \left(q_{k}-q_{k+1}\right), \quad T_{j}=e^{2 \mathrm{i} q_{j}} \quad(\forall j=1, \ldots, n),
$$

then the following Iwasawa decomposition is valid:

$$
g_{k}(\gamma) \mathcal{N}(T) a=b \chi_{k}(\gamma) \quad \text { with } \quad b \in B, \quad b_{j, j}=\hat{a}_{j} \quad(\forall j=1, \ldots, n),
$$


where $\hat{a} \in A$ is as claimed by the lemma. To finish the proof, we notice from (4.16) that $\Xi_{R}\left(g_{k}(\gamma) \Lambda_{L}(K)\right)=\chi_{k}(\gamma)^{-1}$, and this allows us to calculate (cf. (2.35)) that

$$
\Xi_{R}\left(g_{k}(\gamma) \triangleright K\right)=\sigma_{k, k+1}(T)
$$

By the second sentence of Lemma 4.1, this implies that $g_{k}(\gamma) \triangleright K \in \tilde{S}$. Therefore the element $\left[g\left(K, \sigma_{k, k+1}\right)\right]$ of Lemma 4.2 is provided by $g_{k}(\gamma)$ with $\gamma$ in (4.15). Since any $K \in \tilde{S}$ is determined by $\Xi_{R}(K)$ and the diagonal part of $\Lambda_{L}(K)$, it follows that $b$ in (4.16) is given by $b=\mathcal{N}\left(\sigma_{k, k+1}(T)\right) \hat{a}$, which can be checked also by direct calculation. Q.E.D.

Lemma 4.4. The image of the map $\tilde{\mathcal{I}}$ defined in Theorem 3.1 is the submanifold $\tilde{S}$ defined in (4.2). The corresponding map $\tilde{\mathcal{I}}: T^{*} \mathbb{T}_{n}^{0} \rightarrow \tilde{S}$ is an $S(n)$-equivariant symplectic diffeomorphism. Here, we refer to the $S(n)$-action on $\left(T^{*} \mathbb{T}_{n}^{0}, \Omega_{T^{*} \mathbb{T}_{n}^{0}}\right)$ obtained as the cotangent lift of the permutation action on $\mathbb{T}_{n}^{0}$ and to the $S(n)$-action on $\left(\tilde{S}, \omega_{\tilde{S}}\right)$ described in Lemmas 4.2 and 4.3.

Proof. It follows from the definition of $\tilde{\mathcal{I}}$ in Theorem 3.1 and from equation (4.1) that we have the equality

$$
\tilde{\mathcal{I}}\left(e^{2 \mathrm{i} q}, p\right)=\mathcal{N}\left(e^{2 \mathrm{i} q}\right) a\left(e^{2 \mathrm{i} q}, p\right) e^{-2 \mathrm{i} q}
$$

with the function $a=\operatorname{diag}\left(a_{1}, \ldots, a_{n}\right)$ given by

$$
a_{j}\left(e^{2 \mathrm{i} q}, p\right):=e^{-\frac{p_{j}}{2}} \prod_{m<j}\left[1+\frac{\sinh ^{2} \frac{x}{2}}{\sin ^{2}\left(q_{j}-q_{m}\right)}\right]^{-\frac{1}{4}} \prod_{m>j}\left[1+\frac{\sinh ^{2} \frac{x}{2}}{\sin ^{2}\left(q_{j}-q_{m}\right)}\right]^{\frac{1}{4}}, \quad j=1, \ldots, n
$$

By taking into account the identification (3.17) and the definition (4.2), this ensures the validity of the first sentence of the lemma. One can see from the formula of $\tilde{\mathcal{I}}$ or directly from Lemma 4.1 that $\tilde{\mathcal{I}}: T^{*} \mathbb{T}_{n}^{0} \rightarrow \tilde{S}$ is a diffeomorphism. The symplectic property $\tilde{\mathcal{I}}^{*} \omega_{\tilde{S}}=\Omega_{T^{*} \mathbb{T}_{n}^{0}}$ (with (3.18) and (4.4) ) can be established directly. In fact, the properties mentioned so far would hold also if one replaced $\left[1+\left(\sinh ^{2} \frac{x}{2}\right) / \sin ^{2}\left(q_{j}-q_{m}\right)\right]$ in (4.19) by any positive even function $W\left(q_{j}-q_{m}\right)$.

It is sufficient to confirm the equivariance property of $\tilde{\mathcal{I}}$ for the transpositions

$$
\sigma:=\sigma_{k, k+1} \in S(n), \quad k=1, \ldots, n-1 .
$$

From (4.18) we obtain

$$
\tilde{\mathcal{I}}\left(\sigma\left(e^{2 \mathrm{i} q}\right), \sigma(p)\right)=\mathcal{N}\left(\sigma\left(e^{2 \mathrm{i} q}\right)\right) a\left(\sigma\left(e^{2 \mathrm{i} q}\right), \sigma(p)\right)\left(\sigma\left(e^{2 \mathrm{i} q}\right)\right)^{-1} .
$$

For any fixed $k$, is easily checked that $a_{j}\left(\sigma\left(e^{2 \mathrm{i} q}\right), \sigma(p)\right)=a_{j}\left(e^{2 \mathrm{i} q}, p\right)$ if $j \notin\{k, k+1\}$ and

$$
a_{k}\left(\sigma\left(e^{2 \mathrm{i} q}\right), \sigma(p)\right)=a_{k+1}\left(e^{2 \mathrm{i} q}, p\right) W_{k}\left(e^{2 \mathrm{i} q}\right), \quad a_{k+1}\left(\sigma\left(e^{2 \mathrm{i} q}\right), \sigma(p)\right)=\frac{a_{k}\left(e^{2 \mathrm{i} q}, p\right)}{W_{k}\left(e^{2 \mathrm{i} q}\right)}
$$

with the same function $W_{k}$ as in (4.10). The comparison of (4.21) with (4.9) shows that the proof is complete. Q.E.D.

Our lemmas explain the geometric picture behind Theorem 3.1, which is now easy to prove. 
Proof of Theorem 3.1: Lemmas 4.1 and 4.2 give rise to the identification of symplectic manifolds

$$
\tilde{S} / S(n) \simeq F_{\nu(x)} / \bar{G}_{\nu(x)} .
$$

Here, $\tilde{S} / S(n)$ is the space of orbits of the $S(n)$-action given by Lemma 4.2 , its symplectic form descends from $\omega_{\tilde{S}}$ on $\tilde{S}$, while $\left(F_{\nu(x)} / \bar{G}_{\nu(x)}, \Omega_{\nu(x)}\right)$ is the reduced phase space of interest. Moreover, we constructed the following commutative diagram of maps:

$$
\begin{array}{ccc}
T^{*} \mathbb{T}_{n}^{0} & \stackrel{\tilde{\mathcal{I}}}{\longrightarrow} & \tilde{S} \\
\pi_{1} \downarrow & \downarrow \pi_{\tilde{S}} \\
T^{*} Q(n) & \stackrel{\mathcal{I}}{\longrightarrow} & \tilde{S} / S(n)
\end{array}
$$

The map $\mathcal{I}$ is well-defined by this diagram and is a diffeomorphism, because of Lemma 4.4. (To compare with (3.24), note that $\pi_{\tilde{S}} \circ \tilde{\mathcal{I}}=\pi \circ \tilde{\mathcal{I}}$ since the image of $\tilde{\mathcal{I}}$ is $\tilde{S} \subset F_{\nu}(x)$.) We also established the relation (3.19) as well as $\pi_{\tilde{S}}^{*}\left(\Omega_{\nu(x)}\right)=\omega_{\tilde{S}}$ (by (4.23)) and $\tilde{\mathcal{I}}^{*}\left(\omega_{\tilde{S}}\right)=\Omega_{T^{*} \mathbb{T}_{n}^{0}}$ (by Lemma 4.4). These relations and the fact that $\pi_{1}$ and $\pi_{\tilde{S}}$ are local diffeomorphisms imply that $\mathcal{I}^{*}\left(\Omega_{\nu(x)}\right)=\Omega_{T^{*} Q(n)}$. Q.E.D.

Proof of Theorem 3.2: Denote by $\mathcal{L}^{\tilde{S}}$ the restriction of the unreduced Lax matrix $\mathcal{L}(2.60)$ to $\tilde{S}(4.2)$. The definition directly yields the formula

$$
\mathcal{L}^{\tilde{S}}=T a^{-1} \mathcal{N}(T)^{-1}\left(\mathcal{N}(T)^{-1}\right)^{\dagger} a^{-1} T^{-1},
$$

where $T, a$ and $\mathcal{N}(T)$ are understood as evaluation functions on $\tilde{S} \simeq \mathbb{T}_{n}^{0} \times A$. Next, we remark that the restriction of the moment map constraint (3.14) to $\tilde{S}$ is equivalent to the relation

$$
\mathcal{N}(T) a \mathcal{L}^{\tilde{S}}(\mathcal{N}(T) a)^{\dagger}=\nu(x) \nu(x)^{\dagger}=e^{-x}\left[\mathbf{1}_{n}+\frac{e^{n x}-1}{n} v(x) v(x)^{\dagger}\right]
$$

which can be rewritten as

$$
e^{\frac{x}{2}} \mathcal{L}_{j k}^{\tilde{S}}-e^{-\frac{x}{2}} T_{j}^{-1} \mathcal{L}_{j k}^{\tilde{S}} T_{k}=2 \mathcal{U}_{j} \overline{\mathcal{U}}_{k} a_{j}^{-1} a_{k}^{-1} \sinh \frac{x}{2},
$$

if we define

$$
\mathcal{U}_{j}:=\left[\frac{e^{-\frac{x}{2}}\left(e^{n x}-1\right)}{2 n \sinh \frac{x}{2}}\right]^{\frac{1}{2}}\left(\mathcal{N}(T)^{-1} v(x)\right)_{j}:=\eta_{j}\left|\mathcal{U}_{j}\right|
$$

Here, $T=\operatorname{diag}\left(T_{1}, \ldots, T_{n}\right)$ and $a=\operatorname{diag}\left(a_{1}, \ldots, a_{n}\right)$. By solving (4.27) for $\mathcal{L}^{\tilde{S}}$ we arrive at

$$
\mathcal{L}_{j k}^{\tilde{S}}=\eta_{j} \frac{2 a_{j}^{-1}\left|\mathcal{U}_{j}\right| a_{k}^{-1}\left|\mathcal{U}_{k}\right| \sinh \frac{x}{2}}{e^{\frac{x}{2}}-e^{-\frac{x}{2}} T_{j}^{-1} T_{k}} \eta_{k}^{-1} .
$$

With the inverse $\mathcal{N}(T)^{-1}$ displayed in [11], it is also straightforward to calculate that

$$
\left|\mathcal{U}_{j}\right|=\prod_{m>j}\left[1+\frac{\sinh ^{2} \frac{x}{2}}{\sin ^{2}\left(q_{j}-q_{m}\right)}\right]^{\frac{1}{2}}
$$


where we use the parametrization $T_{k}=e^{2 \mathrm{i} q_{k}}$ for all $k=1, \ldots, n$. Next, let us parametrize $a \in A$ according to (4.19) and insert also (4.30) into (4.29). Then we obtain

$$
\mathcal{L}_{j k}^{\tilde{S}}=\frac{\gamma_{j} \gamma_{k}^{-1} e^{\frac{p_{j}+p_{k}}{2}} \sinh \frac{x}{2}}{\sinh \left(\frac{x}{2}+\mathrm{i} q_{j}-\mathrm{i} q_{k}\right)} \prod_{m \neq j}\left[1+\frac{\sinh ^{2} \frac{x}{2}}{\sin ^{2}\left(q_{j}-q_{m}\right)}\right]^{\frac{1}{4}} \prod_{s \neq k}\left[1+\frac{\sinh ^{2} \frac{x}{2}}{\sin ^{2}\left(q_{k}-q_{s}\right)}\right]^{\frac{1}{4}}
$$

with $\gamma_{j}:=\eta_{j} e^{\mathrm{i} q_{j}}$. Hence $\mathcal{L}^{\tilde{S}}$ is conjugate to the standard Ruijsenaars-Schneider Lax matrix $L$ in (1.1).

Recall from (4.18) that the map $\tilde{\mathcal{I}}: T^{*} \mathbb{T}_{n}^{0} \rightarrow F_{\nu(x)}$ in (3.21), (3.22) was obtained from the map in (4.3) by using the identification $T^{*} \mathbb{T}_{n}^{0} \simeq \mathbb{T}_{n}^{0} \times \mathbb{R}^{n}$ (3.17) and the parametrization (4.19) of $a_{j}$. Therefore the foregoing arguments prove Theorem 3.2. Q.E.D.

Remark 4.1. The statement of Theorem 3.2 was also obtained in [11, but there the details of the proof were omitted for lack of space. The 'useful substitution' (4.19) was introduced in [11 (Eq. (3.13) in loc. cit.) just on the basis that it converts the expression $T a^{-1} \mathcal{N}(T)^{-1}\left(\mathcal{N}(T)^{-1}\right)^{\dagger} a^{-1} T^{-1}$ (4.25) into a conjugate of the standard Lax matrix $L(q, p)$ (1.1). The deeper geometric meaning of this substitution is now revealed by Lemma 4.4 above. Note that $\mathcal{L}^{\tilde{S}}$ transforms by conjugation under the 'residual gauge transformations' of Lemma 4.2, and this corresponds to the fact that $L(q, p)$, viewed as a function on $T^{*} \mathbb{T}_{n}^{0}$ (3.17), transforms by conjugation under the natural $S(n)$-action.

Remark 4.2. The fact that $T^{*} Q(n)$ appears as a factor space (4.24), and not directly as a global gauge slice, reflects the fact that $Q(n)$ is not a submanifold of $\mathbb{T}_{n}^{0}$ (see also Appendix B).

Remark 4.3. It is worth pointing out that the consequence (4.27) of the moment map constraint is essentially identical to the 'commutation relation of the Lax matrix' that played an important rôle in the analysis presented in [30].

\section{$5 \quad$ Proofs of Theorems 3.3 and 3.4}

The proofs of Theorems 3.3 and 3.4 will be based on a series of preliminary lemmas. From now on we adopt the identification $\hat{p} \simeq \operatorname{diag}\left(\hat{p}_{1}, \ldots, \hat{p}_{n}\right)$ for any $\hat{p} \in \overline{\mathfrak{C}}_{0}(\underline{3.26})$.

Lemma 5.1. Every element $K$ of the group $G L(n, \mathbb{C})$ can be decomposed as

$$
K=k_{L} \triangleright\left(e^{-\hat{p}} k_{R}^{-1}\right), \quad k_{L}, k_{R} \in U(n), \quad \hat{p} \in \overline{\mathfrak{C}}_{0} .
$$

Moreover, if $\tau$ is any element of the maximal torus $\mathbb{T}_{n}<U(n)$ then the triple $k_{L} \tau, \hat{p}, \tau^{-1} k_{R} \tau$ gives the same element $K$ as the triple $k_{L}, \hat{p}, k_{R}$, and this is the maximal possible ambiguity of the decomposition (5.1) if $\hat{p}$ is regular $\left(\hat{p}_{i}>\hat{p}_{i+1}, \forall i=1, \ldots, n-1\right)$.

Proof. The statement of the lemma is a direct consequence of the standard Cartan decomposition of the elements of $G L(n, \mathbb{C})$. Indeed, it is well known that every element $K$ of the group $G L(n, \mathbb{C})$ can be decomposed as

$$
K=\eta_{L} e^{-\hat{p}} \eta_{R}^{-1}, \quad \eta_{L}, \eta_{R} \in U(n), \quad \hat{p} \in \overline{\mathfrak{C}}_{0} .
$$


For each $K$, the diagonal matrix $\hat{p}$ in the standard Cartan decomposition (5.2) is defined unambiguously. Moreover, simultaneous right multiplication of the pair $\eta_{L}, \eta_{R}$ by an element $\tau$ of the maximal torus $\mathbb{T}_{n}$ gives an equally good pair $\eta_{L} \tau, \eta_{R} \tau$ and this is the maximal possible ambiguity of the decomposition (5.2) if $\hat{p}$ is regular.

From (5.2) and the definition (2.21) of the quasi-adjoint action, we obtain

$$
K=k_{L} \triangleright\left(e^{-\hat{p}} k_{R}^{-1}\right), \quad k_{L}, k_{R} \in U(n),
$$

where

$$
k_{L}=\eta_{L}, \quad k_{R}=\Xi_{R}\left(\eta_{L} e^{-\hat{p}}\right) \eta_{R}
$$

The proof is finished by noting that $\left(\eta_{L} \tau, \eta_{R} \tau\right)$ corresponds by (5.4) to $\left(k_{L} \tau, \tau^{-1} k_{R} \tau\right)$ for all $\tau \in \mathbb{T}_{n}$. Q.E.D.

Lemma 5.2. If $K=k_{L} \triangleright\left(e^{-\hat{p}} k_{R}^{-1}\right)$ (5.1) is a solution of the moment map constraint (3.14) then $\hat{p} \in \overline{\mathfrak{C}}_{x}$, as defined in (3.26).

Proof. By using the formula

$$
\Lambda_{L}(K)=\Lambda_{R}\left(K^{-1}\right), \quad \forall K \in G L(n, \mathbb{C}),
$$

we can rewrite the moment map constraint (3.14) as

$$
\Lambda\left(k_{L} \triangleright\left(e^{-\hat{p}} k_{R}^{-1}\right)\right)=\Lambda_{L}\left(k_{L} e^{-\hat{p}}\right) \Lambda_{L}\left(\Xi_{R}^{-1}\left(k_{L} e^{-\hat{p}}\right) k_{R} e^{\hat{p}}\right)=\nu(x),
$$

or, equivalently, as

$$
\Lambda\left(k_{L} \triangleright\left(e^{-\hat{p}} k_{R}^{-1}\right)\right) \Lambda\left(k_{L} \triangleright\left(e^{-\hat{p}} k_{R}^{-1}\right)\right)^{\dagger}=k_{L} e^{-\hat{p}} k_{R} e^{2 \hat{p}} k_{R}^{-1} e^{-\hat{p}} k_{L}^{-1}=\nu(x) \nu(x)^{\dagger} .
$$

By means of (3.11), the last equality can be further rewritten as

$$
k_{R} e^{2 \hat{p}} k_{R}^{-1}=\left[e^{2 \hat{p}} e^{-x}+e^{-x} \frac{e^{n x}-1}{n} e^{\hat{p}} k_{L}^{-1} v(x) v(x)^{\dagger} k_{L} e^{\hat{p}}\right] .
$$

The equality of the characteristic polynomials of the matrices on the two sides of (5.8) gives

$$
\prod_{j}\left(e^{2 \hat{p}_{j}}-\lambda\right)=\prod_{j}\left(e^{2 \hat{p}_{j}-x}-\lambda\right)+e^{-x} \frac{e^{n x}-1}{n} \sum_{j}\left(e^{2 \hat{p}_{j}}\left|w_{j}\right|^{2} \prod_{k \neq j}\left(e^{2 \hat{p}_{k}-x}-\lambda\right)\right),
$$

where

$$
w:=k_{L}^{-1} v(x)
$$

and $\lambda$ is a complex variable. To derive (5.9), we used the identity

$$
\operatorname{det}\left(\mathbf{1}_{n}+u y^{\dagger}\right)=1+y^{\dagger} u
$$

which is valid for arbitrary $n$-component column vectors $u$ and $y$. 
Suppose that (5.8) holds for some regular $K$, i.e., $\hat{p}_{1}>\hat{p}_{2}>\ldots>\hat{p}_{n}$. We can then evaluate the polynomials on both sides of (5.9) at the $n$ different values $\lambda=e^{2 \hat{p}_{j}-x}, j=1, \ldots, n$. This yields

$$
\left|w_{j}\right|^{2}=n \frac{1-e^{-x}}{1-e^{-n x}} \prod_{k \neq j} \frac{1-e^{2 \hat{p}_{j}-2 \hat{p}_{k}-x}}{1-e^{2 \hat{p}_{j}-2 \hat{p}_{k}}}, \quad j=1, \ldots, n .
$$

Consider first the case $x>0$. On account of $\hat{p}_{1}>\hat{p}_{2}>\ldots>\hat{p}_{n}$, we find from (5.12) for each $j=1, \ldots, n-1$ the following inequality

$$
\prod_{k>j}\left(e^{2 \hat{p}_{j}-2 \hat{p}_{k}-x}-1\right)=\frac{1-e^{-n x}}{1-e^{-x}} \frac{\left|w_{j}\right|^{2}}{n} \prod_{k>j}\left(e^{2 \hat{p}_{j}-2 \hat{p}_{k}}-1\right) \prod_{k<j} \frac{1-e^{2 \hat{p}_{j}-2 \hat{p}_{k}}}{1-e^{2 \hat{p}_{j}-2 \hat{p}_{k}-x}} \geq 0 .
$$

Now we prove by induction that

$$
\hat{p}_{l}-\hat{p}_{l+1}-\frac{x}{2} \geq 0, \quad \forall l=1, \ldots, n-1 .
$$

First of all, for $j=n-1$, the inequality (5.13) gives immediately (5.14) for $l=n-1$. It is easy to see that if (5.14) holds for $l=j+1, j+2, \ldots, n-1$, then it holds also for $l=j$. Indeed, this follows from

$$
0 \leq \prod_{k>j}\left(e^{2 \hat{p}_{j}-2 \hat{p}_{k}-x}-1\right)=\left(e^{2 \hat{p}_{j}-2 \hat{p}_{j+1}-x}-1\right) \prod_{k>j+1}\left(e^{2 \hat{p}_{j}-2 \hat{p}_{k-1}+2 \hat{p}_{k-1}-2 \hat{p}_{k}-x}-1\right) .
$$

The case $x<0$ is very similar. The point of departure is the following inequality

$$
\prod_{k<j}\left(1-e^{2 \hat{p}_{j}-2 \hat{p}_{k}-x}\right)=\frac{e^{-n x}-1}{e^{-x}-1} \frac{\left|w_{j}\right|^{2}}{n} \prod_{k<j}\left(1-e^{2 \hat{p}_{j}-2 \hat{p}_{k}}\right) \prod_{k>j} \frac{e^{2 \hat{p}_{j}-2 \hat{p}_{k}}-1}{e^{2 \hat{p}_{j}-2 \hat{p}_{k}-x}-1} \geq 0, \quad \forall j=2, \ldots, n .
$$

In this case we prove by induction that

$$
\hat{p}_{l-1}-\hat{p}_{l}+\frac{x}{2} \geq 0, \quad \forall l=2, \ldots, n .
$$

Now (5.16) for $j=2$ gives (5.17) for $l=2$. By using

$$
0 \leq \prod_{k<j}\left(1-e^{2 \hat{p}_{j}-2 \hat{p}_{k}-x}\right)=\left(1-e^{2 \hat{p}_{j}-2 \hat{p}_{j-1}-x}\right) \prod_{k<j-1}\left(1-e^{2 \hat{p}_{j}-2 \hat{p}_{k+1}+2 \hat{p}_{k+1}-2 \hat{p}_{k}-x}\right),
$$

one sees that if (5.17) holds for $l=2, \ldots, j-1$, then it holds also for $l=j$.

So far we have proved Lemma 5.2 for the regular solutions of the moment map constraint (3.14), i.e., for those $K$ (5.1) for which $\hat{p}_{1}>\hat{p}_{2}>\ldots>\hat{p}_{n}$. We remark that such regular solutions exist. As an example, consider $K=k_{L} \triangleright\left(e^{-\hat{p}} k_{R}^{-1}\right)$ with $\hat{p}$ such that

$$
\hat{p}_{l}-\hat{p}_{l+1}=\frac{|x|}{2}, \quad \forall l=1, \ldots, n-1 .
$$

In fact, a solution is then provided by $k_{L}:=\kappa_{L}(x)$ (given by (3.31)) and $k_{R}:=\kappa_{R}(x)$, where, for $x>0$

$$
\kappa_{R}(x)_{n 1}=\kappa_{R}(x)_{i, i+1}(x)=1, \quad i=1, \ldots, n-1, \quad \kappa_{R}(x)_{i j}=0 \quad \text { otherwise }
$$


and for $x<0$

$$
\kappa_{R}(x):=\kappa_{R}(-x)^{-1}
$$

To finish the proof, it remains to treat the case of non-regular solutions of (3.14), for which two or more $\hat{p}_{j}$ 's are equal to each other. Suppose that such a non-regular solution, $K_{0}$, exists. Note that the space of solutions of (3.14), $F_{\nu(x)}$, is connected, since it is the total space of a principal fiber bundle with connected structure group and connected base, as follows from Lemma 3.2 and Theorem 3.1. Then take a regular solution, $K_{1}$, e.g. the one exhibited above, and connect $K_{0}$ with $K_{1}$ by a continuous path $K_{s}, s \in[0,1]$, in $F_{\nu(x)}$. Now the diagonal matrix $\hat{p}(s)$ in the modified Cartan decomposition (5.1) of $K_{s}$ (or, in other words, the spectrum of the element $\sqrt{K_{s} K_{s}^{\dagger}}$ ) varies continuously with $s$. However, this is not possible because the set of the non-regular elements of $\overline{\mathfrak{C}}_{0}$ is disconnected from $\overline{\mathfrak{C}}_{x}$ for $x \neq 0$. Hence, non-regular solutions of the constraint (3.14) do not exist. Q.E.D.

Lemma 5.3. If $K=k_{L} \triangleright\left(e^{-\hat{p}} k_{R}^{-1}\right)$ (5.1) is a solution of the moment map constraint (3.14) then the matrix $k_{R}$ must have the form

$$
k_{R}=\delta_{l} \theta(x, \hat{p}) \delta_{r} .
$$

Here $\delta_{l}, \delta_{r}$ are some diagonal unitary matrices and $\theta(x, \hat{p})$ is the real orthogonal matrix defined for every $\hat{p} \in \overline{\mathfrak{C}}_{x}$ by

$$
\begin{gathered}
\theta(x, \hat{p})_{j k}:=\frac{\sinh \left(\frac{x}{2}\right)}{\sinh \left(\hat{p}_{k}-\hat{p}_{j}\right)} \prod_{m \neq j, k}\left[\frac{\sinh \left(\hat{p}_{j}-\hat{p}_{m}-\frac{x}{2}\right) \sinh \left(\hat{p}_{k}-\hat{p}_{m}+\frac{x}{2}\right)}{\sinh \left(\hat{p}_{j}-\hat{p}_{m}\right) \sinh \left(\hat{p}_{k}-\hat{p}_{m}\right)}\right]^{\frac{1}{2}}, j \neq k, \\
\theta(x, \hat{p})_{j j}:=\prod_{m \neq j}\left[\frac{\sinh \left(\hat{p}_{j}-\hat{p}_{m}-\frac{x}{2}\right) \sinh \left(\hat{p}_{j}-\hat{p}_{m}+\frac{x}{2}\right)}{\sinh ^{2}\left(\hat{p}_{j}-\hat{p}_{m}\right)}\right]^{\frac{1}{2}} .
\end{gathered}
$$

Proof. Consider the following variant of the moment map constraint (5.8):

$$
k_{R}(x) e^{2 \hat{p}} e^{\frac{x}{2}} k_{R}(x)^{-1}=e^{2 \hat{p}} e^{-\frac{x}{2}}+2\left(\sinh \frac{x}{2}\right) \xi(x) \xi(x)^{\dagger},
$$

where the vector $\xi(x)$ is defined as

$$
\xi(x):=\sqrt{\frac{e^{n x}-1}{n\left(e^{x}-1\right)}} e^{\hat{p}} k_{L}^{-1} v(x) .
$$

Here, our notation emphasizes the dependence of $k_{R}$ and $\xi$ on $x$ while their dependence on $\hat{p}$ remains tacit. Observe from the comparison of (5.10), (5.12) and (5.26) that

$$
\left|\xi(x)_{j}\right|^{2}=e^{2 \hat{p}_{j}} \prod_{k \neq j} \frac{e^{x}-e^{2 \hat{p}_{j}-2 \hat{p}_{k}}}{1-e^{2 \hat{p}_{j}-2 \hat{p}_{k}}} .
$$

For any given $\hat{p} \in \overline{\mathfrak{C}}_{x}$ and $\xi(x)$ subject to (5.27), the constraint (5.25) admits a solution for $k_{R}(x)$, since the characteristic polynomials of the matrices on the two sides of (5.25) are equal. 
The solution $k_{R}(x)$ can be chosen to be unitary because the matrix on the right hand side of (5.25) is Hermitian. Suppose that a pair $\left(k_{R}(x), \xi(x)\right) \in U(n) \times \mathbb{C}^{n}$ satisfies (5.25), at some fixed $\hat{p} \in \overline{\mathfrak{C}}_{x}$. Then all pairs satisfying (5.25) can be obtained by replacing the given solution by $\left(\delta_{l} k_{R}(x) \delta_{r}, \delta_{l} \xi(x)\right)$ with arbitrary $\delta_{l}, \delta_{r} \in \mathbb{T}_{n}$, since (5.25) fixes the vector $\xi(x)$ up to phases, according to (5.27), and the eigenvalues of $e^{2 \hat{p}} e^{\frac{x}{2}}$ are distinct.

Let us rearrange the constraint (5.25) as

$$
k_{R}(x)^{-1} e^{2 \hat{p}} e^{-\frac{x}{2}} k_{R}(x)=e^{2 \hat{p}} e^{\frac{x}{2}}-2\left(\sinh \frac{x}{2}\right) k_{R}(x)^{-1} \xi(x) \xi(x)^{\dagger} k_{R}(x) .
$$

Let us also consider (5.25) for $x \rightarrow-x$,

$$
k_{R}(-x) e^{2 \hat{p}} e^{-\frac{x}{2}} k_{R}(-x)^{-1}=e^{2 \hat{p}} e^{\frac{x}{2}}-2\left(\sinh \frac{x}{2}\right) \xi(-x) \xi(-x)^{\dagger} .
$$

By comparing (5.28) and (5.29), we conclude that

$$
\left(k_{R}(x)^{-1} \xi(x)\right)_{j}=e^{\mathrm{i} \eta_{j}} \xi(-x)_{j}
$$

where the $e^{\mathrm{i} \eta_{j}}$ are some phases. Indeed, this follows from the fact that the constraint (5.29) determines the components of $\xi(-x)$ up to phases.

Let us multiply the constraint (5.25) from the right by $k_{R}(x)$. This gives

$$
k_{R}(x) e^{2 \hat{p}} e^{\frac{x}{2}}-e^{2 \hat{p}} e^{-\frac{x}{2}} k_{R}(x)=2\left(\sinh \frac{x}{2}\right) \xi(x) \xi(x)^{\dagger} k_{R}(x) .
$$

Next, by spelling out (5.31) in components and taking into account (5.30), we obtain

$$
\left(e^{2 \hat{p}_{l}+\frac{x}{2}}-e^{2 \hat{p}_{j}-\frac{x}{2}}\right) k_{R}(x)_{j l}=2\left(\sinh \frac{x}{2}\right) \xi(x)_{j} \xi(-x)_{l}^{\dagger} e^{-\mathrm{i} \eta_{l}} .
$$

For any $\hat{p} \in \mathfrak{C}_{x}$, we can deduce from (5.32) that

$$
k_{R}(x)_{j l}=2\left(\sinh \frac{x}{2}\right) \frac{e^{-\mathrm{i} \eta_{l}} \xi(x)_{j} \xi(-x)_{l}^{\dagger}}{e^{2 \hat{p}_{l}+\frac{x}{2}}-e^{2 \hat{p}_{j}-\frac{x}{2}}} .
$$

Now we note that if $\hat{p} \in \mathfrak{C}_{x}$, then $\theta(x, \hat{p})$, defined by (5.23) and (5.24), can be also rewritten as

$$
\theta(x, \hat{p})_{j l}=2\left(\sinh \frac{x}{2}\right) \frac{\left|\xi(x)_{j}\right|\left|\xi(-x)_{l}\right|}{e^{2 \hat{p}_{l}+\frac{x}{2}}-e^{2 \hat{p}_{j}-\frac{x}{2}}}
$$

where the absolute values of the components of the vectors $\xi( \pm x)$ are given by (5.27). It is clear from (5.33) and (5.34) that that each unitary solution $k_{R}(x)$ of (5.25) verifies

$$
k_{R}(x)=\delta_{l} \theta(x, \hat{p}) \delta_{r} \quad \text { with some } \quad \delta_{l}, \delta_{r} \in \mathbb{T}_{n}, \quad \forall \hat{p} \in \mathfrak{C}_{x},
$$

which proves (5.22) for $\hat{p} \in \mathfrak{C}_{x}$. Moreover, the formula in (5.35) entails that the real matrix $\theta(x, \hat{p})$ must be itself unitary, and hence orthogonal, and it must also satisfy (5.25) for all 
$\hat{p} \in \mathfrak{C}_{x}$. By the continuity of $\theta(x, \hat{p})$ as a function of $\hat{p} \in \overline{\mathfrak{C}}_{x}$, it then follows that $\theta(x, \hat{p})$ must be an orthogonal matrix for all $\hat{p} \in \overline{\mathfrak{C}}_{x}$, and there must exists also a vector, say $\tilde{\xi}(x, \hat{p})$, such that

$$
\theta(x, \hat{p}) e^{2 \hat{p}} e^{\frac{x}{2}} \theta(x, \hat{p})^{-1}=e^{2 \hat{p}} e^{-\frac{x}{2}}+2\left(\sinh \frac{x}{2}\right) \tilde{\xi}(x, \hat{p}) \tilde{\xi}(x, \hat{p})^{\dagger}, \quad \forall \hat{p} \in \overline{\mathfrak{C}}_{x} .
$$

Indeed, the fact that $\left(\theta(x, \hat{p}) e^{2 \hat{p}} e^{\frac{x}{2}} \theta(x, \hat{p})^{-1}-e^{2 \hat{p}} e^{-\frac{x}{2}}\right) /\left(2 \sinh \frac{x}{2}\right)$ is a rank-one projector for all $\hat{p} \in \mathfrak{C}_{x}$ implies that the same statement holds also at the boundary of $\overline{\mathfrak{C}}_{x}$. (The rank cannot decrease at the boundary, since the vectors $\xi(x)$ satisfying (5.25) cannot vanish at any $\hat{p} \in \overline{\mathfrak{C}}_{x}$.) Thus we have shown that $\theta(x, \hat{p})$ is unitary and solves (5.25) for all $\hat{p} \in \overline{\mathfrak{C}}_{x}$. By the remarks given after (5.27), this guarantees the validity of (5.22) at every $\hat{p} \in \overline{\mathfrak{C}}_{x}$. We note in passing that, since the vector $\tilde{\xi}(x, \hat{p})$ is determined by $(5.36)$ up to an overall phase at any fixed $\hat{p}$, and $\theta(x, \hat{p})$ is real and continuous, the components of $\tilde{\xi}(x, \hat{p})$ can be chosen to be real, continuous functions of $\hat{p} \in \overline{\mathfrak{C}}_{x}$. Q.E.D.

Lemma 5.4. If $K=k_{L} \triangleright\left(e^{-\hat{p}} k_{R}^{-1}\right)$ (5.1) is a solution of the moment map constraint (3.14) then the matrix $k_{L}$ must have the form

$$
k_{L}=h \kappa_{L}(x) \zeta(x, \hat{p})^{-1} \delta^{-1} .
$$

Here $h \in G_{v(x)}$ (3.13), $\delta$ is some diagonal unitary matrix, the matrix $\kappa_{L}(x)$ is given by (3.31), and $\zeta(x, \hat{p})$ is the real orthogonal matrix defined for every $\hat{p} \in \overline{\mathfrak{C}}_{x}$ by

$\zeta(x, \hat{p})_{a a}=r(x, \hat{p})_{a}, \zeta(x, \hat{p})_{i j}=\delta_{i j}-\frac{r(x, \hat{p})_{i} r(x, \hat{p})_{j}}{1+r(x, \hat{p})_{a}}, \zeta(x, \hat{p})_{i a}=-\zeta(x, \hat{p})_{a i}=r(x, \hat{p})_{i}, \quad i, j \neq a$,

where $a=n$ for $x>0, a=1$ for $x<0$ and, for all $x \neq 0$,

$$
r(x, \hat{p})_{j}:=\sqrt{\frac{1-e^{-x}}{1-e^{-n x}}} \prod_{k \neq j} \sqrt{\frac{1-e^{2 \hat{p}_{j}-2 \hat{p}_{k}-x}}{1-e^{2 \hat{p}_{j}-2 \hat{p}_{k}}}}, \quad j=1, \ldots, n .
$$

Proof. First, let us show that the above real matrix $\zeta(x, \hat{p})$ is orthogonal. For this, note that

$$
\sum_{j}\left|r(x, \hat{p})_{j}\right|^{2}=1 \text {. }
$$

This can be deduced from the comparison of (5.39) and (5.12) by using (5.9) for $\lambda=0$. One can then easily check that the columns of the matrix $\zeta(x, \hat{p})$ form an orthonormal system.

We know from Eqs. (5.10), (5.12) and (5.39) that there exists $\delta \in \mathbb{T}_{n}$ such that

$$
k_{L}^{-1} v(x)=\sqrt{n} \delta r(x, \hat{p}),
$$

where the vector $r(x, \hat{p})$ is defined by its components (5.39). On the other hand, the formula (3.31) leads immediately to

$$
\kappa_{L}(x)^{-1} v(x)=\left(\begin{array}{c}
0 \\
\cdot \\
\cdot \\
\cdot \\
0 \\
\sqrt{n}
\end{array}\right) \quad \text { for } x>0 \text { and } \quad \kappa_{L}(x)^{-1} v(x)=\left(\begin{array}{c}
\sqrt{n} \\
0 \\
\cdot \\
\cdot \\
\cdot \\
0
\end{array}\right) \quad \text { for } x<0
$$


For all $x$, we then obtain

$$
\zeta(x, \hat{p}) \kappa_{L}(x)^{-1} v(x)=\sqrt{n} r(x, \hat{p}) .
$$

By combining this with (5.41), we get

$$
\delta^{-1} k_{L}^{-1} v(x)=\zeta(x, \hat{p}) \kappa_{L}(x)^{-1} v(x),
$$

which implies the existence of an element $h$ from the isotropy group $G_{v(x)}$ of the vector $v(x)$ (3.12) such that (5.37) holds. Q.E.D.

Lemma 5.5. For each $\tau=\operatorname{diag}\left(\tau_{1}, \ldots, \tau_{n}\right) \in \mathbb{T}_{n}$ set

$$
\tau_{(x)}:=\operatorname{diag}\left(\tau_{2}, \ldots, \tau_{n}, 1\right) \quad \text { if } \quad x>0, \quad \tau_{(x)}:=\operatorname{diag}\left(1, \tau_{1}, \ldots, \tau_{n-1}\right) \quad \text { if } \quad x<0 .
$$

Using the previous notations, define the map $K_{x}: G_{v(x)} \times \mathbb{T}_{n} \times \overline{\mathfrak{C}}_{x} \rightarrow G L(n, \mathbb{C})$ by

$$
K_{x}(h, \tau, \hat{p}):=\left(h \kappa_{L}(x) \tau_{(x)} \zeta(x, \hat{p})^{-1}\right) \triangleright\left(e^{-\hat{p}} \tau \tau_{(x)}^{-1} \theta(x, \hat{p})^{-1}\right), \quad \forall(h, \tau, \hat{p}) \in G_{v(x)} \times \mathbb{T}_{n} \times \overline{\mathfrak{C}}_{x}
$$

Then the image of the map $K_{x}$ coincides with the submanifold $F_{\nu(x)}=\Lambda^{-1}(\nu(x))$ of $G L(n, \mathbb{C})$.

Proof. Consider $h=\mathbf{1}_{n}, \tau=\mathbf{1}_{n}$ and $\hat{p} \in \overline{\mathfrak{C}}_{x}$. We first wish to show that $K_{x}\left(\mathbf{1}_{n}, \mathbf{1}_{n}, \hat{p}\right)$ solves the constraint (3.14). This statement is equivalent to

$$
\theta(x, \hat{p}) e^{2 \hat{p}} e^{\frac{x}{2}} \theta(x, \hat{p})^{-1}=e^{2 \hat{p}} e^{-\frac{x}{2}}+2\left(\sinh \frac{x}{2}\right) \Xi(x, \hat{p}) \Xi(x, \hat{p})^{\dagger},
$$

where the real vector $\Xi(x, \hat{p})$ is defined as

$$
\Xi(x, \hat{p}):=\sqrt{\frac{e^{n x}-1}{e^{x}-1}} e^{\hat{p}} r(x, \hat{p}), \quad \forall \hat{p} \in \overline{\mathfrak{C}}_{x},
$$

with $r(x, \hat{p})$ in (5.39). This is so simply because (5.43) holds, and the moment map constraint is (5.25) with (5.26).

Let us recall from the proof of Lemma 5.3 that there exists a real vector $\tilde{\xi}(x, \hat{p})$ that satisfies (5.36). We also know that $\tilde{\xi}(x, \hat{p})$ verifies $\left|\tilde{\xi}(x, \hat{p})_{j}\right|_{\tilde{\xi}}=\Xi(x, \hat{p})_{j}$ for all $j$, and is determined by (5.36) up to an overall sign. Notice from (5.27) that $\tilde{\xi}(x, \hat{p})_{n} \neq 0$ holds for $x>0$ and $\tilde{\xi}(x, \hat{p})_{1} \neq 0$ holds for $x<0$, at each $\hat{p} \in \overline{\mathfrak{C}}_{x}$. This fact allows us to fix the sign ambiguity of $\tilde{\xi}(x, \hat{p})$ by requiring that $\tilde{\xi}(x, \hat{p})_{n}=\Xi(x, \hat{p})_{n}$ for $x>0$ and $\tilde{\xi}(x, \hat{p})_{1}=\Xi(x, \hat{p})_{1}$ for $x<0$.

Now we are going to prove that the unique vector $\tilde{\xi}(x, \hat{p})$ specified above actually satisfies

$$
\tilde{\xi}(x, \hat{p})=\Xi(x, \hat{p}), \quad \forall \hat{p} \in \overline{\mathfrak{C}}_{x},
$$

which converts (5.36) into (5.47). We start by noting from Eqs. (5.23) and (5.24) that

$$
\theta(-x, \hat{p})=\theta(x, \hat{p})^{-1} .
$$

Eqs. (5.36) and (5.50) together imply

$$
\theta(-x, \hat{p}) e^{2 \hat{p}} e^{-\frac{x}{2}} \theta(-x, \hat{p})^{-1}=e^{2 \hat{p}} e^{\frac{x}{2}}-2\left(\sinh \frac{x}{2}\right) \theta(x, \hat{p})^{-1} \tilde{\xi}(x, \hat{p}) \tilde{\xi}(x, \hat{p})^{\dagger} \theta(x, \hat{p}) .
$$


This entails

$$
\theta(x, \hat{p})^{-1} \tilde{\xi}(x, \hat{p})= \pm \tilde{\xi}(-x, \hat{p})
$$

where the overall sign will be determined soon. Let us multiply Eq. (5.36) from the right by $\theta(x, \hat{p})$. This gives

$$
\theta(x, \hat{p})(x) e^{2 \hat{p}} e^{\frac{x}{2}}-e^{2 \hat{p}} e^{-\frac{x}{2}} \theta(x, \hat{p})=2\left(\sinh \frac{x}{2}\right) \tilde{\xi}(x, \hat{p}) \tilde{\xi}(x, \hat{p})^{\dagger} \theta(x, \hat{p}) .
$$

Using Eqs. (5.52) and (5.53), we then find easily

$$
\theta(x, \hat{p})_{j l}= \pm 2\left(\sinh \frac{x}{2}\right) \frac{\tilde{\xi}(x, \hat{p})_{j} \tilde{\xi}(-x, \hat{p})_{l}}{e^{2 \hat{p}_{l}+\frac{x}{2}}-e^{2 \hat{p}_{j}-\frac{x}{2}}} .
$$

On the other hand, we know from (5.34) that

$$
\theta(x, \hat{p})_{j l}=2\left(\sinh \frac{x}{2}\right) \frac{\Xi(x, \hat{p})_{j} \Xi(-x, \hat{p})_{l}}{e^{2 \hat{p}_{l}+\frac{x}{2}}-e^{2 \hat{p}_{j}-\frac{x}{2}}} .
$$

Let us restrict $\hat{p}$ to $\mathfrak{C}_{x}$ in the above two equations (although both (5.54) and (5.55) extend from $\mathfrak{C}_{x}$ to $\overline{\mathfrak{C}}_{x}$ by continuity). Comparing Eqs. (5.54) and (5.55) for $j=n, l=1$ and $j=1, l=n$ fixes the positive sign in (5.52) and (5.54). Comparing Eqs. (5.54) and (5.55) for $j=n, l$ arbitrary and $l=1, j$ arbitrary gives then $\tilde{\xi}(x, \hat{p})=\Xi(x, \hat{p})$ for all $\hat{p} \in \mathfrak{C}_{x}$. By the continuity of $\tilde{\xi}(x, \hat{p})$ and $\Xi(x, \hat{p})$ at every $\bar{p} \in \overline{\mathfrak{C}}_{x}$, the claim of Eq. (5.49) then holds everywhere.

In the above we have proved that $K_{x}\left(\mathbf{1}_{n}, \mathbf{1}_{n}, \hat{p}\right)$ solves the moment map constraint (3.14). Notice from (5.42) and (5.45) that

$$
\kappa_{L}(x) \tau_{(x)} \kappa_{L}(x)^{-1} \in G_{v(x)}, \quad \forall \tau \in \mathbb{T}_{n} .
$$

It follows that whenever $k_{L}, \hat{p}$ and $k_{R}$ solve (5.8), then also $h \kappa_{L}(x) \tau_{(x)} \kappa_{L}(x)^{-1} k_{L}, \hat{p}$ and $k_{R} \tau^{-1} \tau_{(x)}$ solve (5.8) for every $h \in G_{v(x)}$ and $\tau \in \mathbb{T}_{n}$. This means that also $K_{x}(h, \tau, \hat{p})$ solves the moment map constraint (3.14).

Let us now show that all solutions of the moment map constraint (3.14) are of the form $K_{x}(h, \tau, \hat{p})$. Using the Lemmas 5.2, 5.3 and 5.4, we know that the most general solution of the moment map constraint must be of the form $k_{L} \triangleright\left(e^{-\hat{p}} k_{R}^{-1}\right)$, where $\hat{p} \in \overline{\mathfrak{C}}_{x}$ and

$$
k_{R}=\delta_{l} \theta(x, \hat{p}) \delta_{r}, \quad k_{L}=h \kappa_{L}(x) \zeta(x, \hat{p})^{-1} \delta^{-1}
$$

with arbitrary $h \in G_{v(x)}$ and certain $\delta_{l}, \delta_{r}, \delta \in \mathbb{T}_{n}$. The substitution of (15.57) into the moment map constraint (5.8) gives

$$
\theta(x, \hat{p}) e^{2 \hat{p}} \theta(x, \hat{p})^{-1}=e^{2 \hat{p}} e^{-x}+e^{-x} \frac{e^{n x}-1}{n} e^{\hat{p}} \delta_{l}^{-1} \delta \zeta(x, \hat{p}) \kappa_{L}(x)^{-1} v(x) v(x)^{\dagger} \kappa_{L}(x) \zeta(x, \hat{p})^{-1} \delta^{-1} \delta_{l} e^{\hat{p}} .
$$

On the other hand, from Eqs. (5.38), (5.42), (5.47) and (5.48), we deduce

$$
\theta(x, \hat{p}) e^{2 \hat{p}} \theta(x, \hat{p})^{-1}=e^{2 \hat{p}} e^{-x}+e^{-x} \frac{e^{n x}-1}{n} e^{\hat{p}} \zeta(x, \hat{p}) \kappa_{L}(x)^{-1} v(x) v(x)^{\dagger} \kappa_{L}(x) \zeta(x, \hat{p})^{-1} e^{\hat{p}} .
$$


The compatibility of Eqs. (5.58) and (5.59) requires that

$$
\delta_{l}^{-1} \delta \zeta(x, \hat{p}) \kappa_{L}(x)^{-1} v(x)=\gamma \zeta(x, \hat{p}) \kappa_{L}(x)^{-1} v(x)
$$

with some phase $\gamma \in U(1)$. This then implies that

$$
\delta_{l}^{-1} \delta \zeta(x, \hat{p}) \kappa_{L}(x)^{-1}=\gamma \zeta(x, \hat{p}) \kappa_{L}(x)^{-1} h^{-1}
$$

with some $h^{\prime} \in G_{v(x)}$. By taking the inverse of this equation, we obtain

$$
\kappa_{L}(x) \zeta(x, \hat{p})^{-1} \delta^{-1} \delta_{l}=h^{\prime} \gamma^{-1} \kappa_{L}(x) \zeta(x, \hat{p})^{-1} .
$$

Finally, we conclude

$$
k_{L} \triangleright\left(e^{-\hat{p}} k_{R}^{-1}\right)=\left[h \kappa_{L}(x) \zeta(x, \hat{p})^{-1} \delta^{-1}\right] \triangleright\left[e^{-\hat{p}} \delta_{r}^{-1} \theta(x, \hat{p})^{-1} \delta_{l}^{-1}\right]=K_{x}\left(h^{\prime \prime}, \aleph\left(x, \delta_{l} \delta_{r}\right), \hat{p}\right),
$$

where

$$
h^{\prime \prime}=h h^{\prime} \kappa_{L}(x) \aleph\left(x, \delta_{l} \delta_{r}\right)_{(x)}^{-1} \kappa_{L}(x)^{-1} \in G_{v(x)}
$$

with

$$
\aleph(x, \tau)_{j}:=\prod_{k=j}^{n} \tau_{k}^{-1}, \quad x>0 \quad \text { and } \quad \aleph(x, \tau)_{j}:=\prod_{k=1}^{j} \tau_{k}^{-1}, \quad x<0 .
$$

The proof is complete by the last equality in (5.63). To derive this, in addition to (5.62) we also employed the identity

$$
\aleph(x, \tau)(\aleph(x, \tau))_{(x)}^{-1}=\tau^{-1}, \quad \forall \tau \in \mathbb{T}_{n},
$$

which is satisfied by the bijection $\aleph(x, \cdot): \mathbb{T}_{n} \rightarrow \mathbb{T}_{n}$ defined by (5.65). Q.E.D.

Recall the following lemma proved in [17] (Lemma 5.1 therein).

Lemma 5.6 ([17]). Let $A<G L(n, \mathbb{C})$ be the subgroup of real diagonal matrices with positive entries. Consider the three maps $\iota, \iota_{L, R}: U(n) \times A \times U(n) \rightarrow G L(n, \mathbb{C})$ defined by

$$
\iota\left(\eta_{L}, a, \eta_{R}\right):=\eta_{L} a \eta_{R}^{-1}, \quad \iota_{L}\left(\eta_{L}, a, \eta_{R}\right):=\eta_{L} a, \quad \iota_{R}\left(\eta_{L}, a, \eta_{R}\right):=a \eta_{R}^{-1} .
$$

Then the $\iota$-pullback and $\iota_{L, R}$-pullbacks of the symplectic form $\omega_{+}$given by (2.8) are related as

$$
\iota^{*} \omega_{+}=\iota_{L}^{*} \omega_{+}+\iota_{R}^{*} \omega_{+}
$$

Lemma 5.7. Using the notations (5.46) and (5.65), define the smooth map $k_{x}$ from the phase space $\hat{P}=\mathbb{T}_{n} \times \mathfrak{C}_{x}$ of the dual trigonometric Ruijsenaars-Schneider system into $G L(n, \mathbb{C})$ by

$$
k_{x}(\hat{q}, \hat{p}):=K_{x}\left(\mathbf{1}_{n}, \aleph\left(x, e^{\mathrm{i} \hat{q}}\right), \hat{p}\right) .
$$

Then it holds

$$
k_{x}^{*} \omega_{+}=\hat{\omega}
$$


where $\hat{\omega}$ is the symplectic form on $\hat{P}$ given by (1.4).

Proof. Define the map $\eta: \mathbb{T}_{n} \times \mathfrak{C}_{x} \rightarrow U(n) \times A \times U(n)$ by

$$
\eta(\tau, \hat{p}):=\left(\eta_{L}(\hat{p}), e^{-\hat{p}}, \eta_{R}(\tau, \hat{p})\right), \quad \forall \tau \in \mathbb{T}_{n}, \quad \forall \hat{p} \in \mathfrak{C}_{x},
$$

where

$$
\eta_{L}(\hat{p}):=\kappa_{L}(x) \zeta(x, \hat{p})^{-1}, \quad \eta_{R}(\tau, \hat{p}):=\Xi_{R}\left(\kappa_{L}(x) \zeta(x, \hat{p})^{-1} e^{-\hat{p}}\right)^{-1} \theta(x, \hat{p}) \tau_{(x)} \tau^{-1} .
$$

It follows from Eqs. (2.21), (5.46) and (5.67) that

$$
K_{x}\left(\mathbf{1}_{n}, \tau, \hat{p}\right)=\left(\kappa_{L}(x) \tau_{(x)} \kappa_{L}(x)^{-1}\right) \triangleright \iota(\eta(\tau, \hat{p})) .
$$

Now we wish to calculate the pull-back $K_{x}\left(\mathbf{1}_{n}\right)^{*} \omega_{+}$where the map $K_{x}\left(\mathbf{1}_{n}\right): \mathbb{T}_{n} \times \mathfrak{C}_{x} \rightarrow G L(n, \mathbb{C})$ is defined by $K_{x}\left(\mathbf{1}_{n}\right)(\tau, \hat{p}):=K_{x}\left(\mathbf{1}_{n}, \tau, \hat{p}\right)$. The factor $\kappa_{L}(x) \tau_{(x)} \kappa_{L}(x)^{-1}$ does not contribute to this pull-back, since $K_{x}\left(\mathbf{1}_{n}\right)$ takes its values in $F_{\nu(x)}$ by Lemma 4.5 and $\kappa_{L}(x) \tau_{(x)} \kappa_{L}(x)^{-1}$ acts by gauge transformations on $F_{\nu(x)}$ because of (5.56). By combining (5.73) with Lemma 4.6, we then obtain

$$
K_{x}\left(\mathbf{1}_{n}\right)^{*} \omega_{+}=\eta^{*} \iota^{*} \omega_{+}=\eta^{*} \iota_{L}^{*} \omega_{+}+\eta^{*} \iota_{R}^{*} \omega_{+}
$$

Next, notice that

$$
\eta^{*} \iota_{L}^{*} \omega_{+}=0
$$

This is because the element $\eta_{L}(\hat{p}) e^{-\hat{p}}$ is real, the images of the Iwasawa maps $\Lambda_{L}, \Lambda_{R}, \Xi_{R}, \Xi_{L}$ are therefore real, too, and hence the imaginary part of the expression under the trace in (2.8) vanishes in this case.

In order to find $\eta^{*} \iota^{*} \omega_{+}=\eta^{*} \iota_{R}^{*} \omega_{+}$, we have to calculate

$$
\Lambda_{L}\left(a \eta_{R}^{-1}\right)=a, \quad \Xi_{R}\left(a \eta_{R}^{-1}\right)=\rho \tau_{(x)} \tau^{-1}, \quad \Xi_{L}\left(a \eta_{R}^{-1}\right)=\tau \tau_{(x)}^{-1} \mu, \quad \Lambda_{R}\left(a \eta_{R}^{-1}\right)=\rho a^{-1} \mu,
$$

where

$$
\rho:=\eta_{R}(\tau, \hat{p}) \tau \tau_{(x)}^{-1}, \quad \mu:=\Xi_{L}\left(e^{-\hat{p}} \rho^{-1}\right) .
$$

By using the formula (2.8), we compute directly

$$
\begin{gathered}
\eta^{*} \iota_{R}^{*} \omega_{+}=\frac{1}{2} \Im \operatorname{tr}\left(d \hat{p} \wedge\left(d \tau_{(x)} \tau_{(x)}^{-1}-d \tau \tau^{-1}-\tau \tau_{(x)}^{-1} d \mu \mu^{-1} \tau_{(x)} \tau^{-1}\right)\right)+ \\
+\frac{1}{2} \Im \operatorname{tr}\left(\left(d \rho \rho^{-1}+\rho d \hat{p} \rho^{-1}+\rho e^{\hat{p}} d \mu \mu^{-1} e^{-\hat{p}} \rho^{-1}\right) \wedge\left(d \rho \rho^{-1}+\rho\left(d \tau_{(x)} \tau_{(x)}^{-1}-d \tau \tau^{-1}\right) \rho^{-1}\right)\right) .
\end{gathered}
$$

By eliminating the terms that vanish due to the fact that the imaginary part of a real number vanishes, it remains

$$
\eta^{*} \iota_{R}^{*} \omega_{+}=\frac{1}{2} \Im \operatorname{tr}\left(\left(2 d \hat{p}+\rho^{-1} d \rho+d \mu \mu^{-1}\right) \wedge\left(d \tau_{(x)} \tau_{(x)}^{-1}-d \tau \tau^{-1}\right)\right) .
$$

Finally, we note that both $\rho$ and $\mu$ are real orthogonal matrices, hence both $\rho^{-1} d \rho$ and $d \mu \mu^{-1}$ are 1-forms taking values in the space of antisymmetric matrices. Since the trace of the product of an antisymmetric matrix with a diagonal matrix vanishes, we arrive at

$$
K_{x}\left(\mathbf{1}_{n}\right)^{*} \omega_{+}=\eta^{*} \iota_{R}^{*} \omega_{+}=\Im \operatorname{tr}\left(d \hat{p} \wedge\left(\tau \tau_{(x)}^{-1} d\left(\tau_{(x)} \tau^{-1}\right)\right)\right) .
$$


With the help of the relation $k_{x}(\hat{q}, \hat{p})=K_{x}\left(\mathbf{1}_{n}\right)\left(\aleph\left(x, e^{\mathrm{i} \hat{q}}\right), \hat{p}\right)$ and (5.66) $)$, we conclude from (5.80) that

$$
k_{x}^{*} \omega_{+}=\sum_{j} d \hat{p}_{j} \wedge d \hat{q}_{j}=\hat{\omega} .
$$

Q.E.D

Proof of Theorem 3.3: First of all we remind that the dual Ruijsenaars-Schneider phase space $\hat{P}$ is identical to $\mathbb{T}_{n} \times \mathfrak{C}_{x}$ as a manifold (cf. Remark 3.4). The map $\mathcal{Z}_{x}: \hat{P} \rightarrow \hat{P}_{c}$, viewed as the map $\mathcal{Z}_{x}: \mathbb{T}_{n} \times \mathfrak{C}_{x} \rightarrow \hat{P}_{c}$, can be extended to a surjective map $\tilde{\mathcal{Z}}_{x}: \mathbb{T}_{n} \times \overline{\mathfrak{C}}_{x} \rightarrow \hat{P}_{c}$ by using the same formulae (3.28) and (3.29) that define $\mathcal{Z}_{x}$. In correspondence with the Cartesian product $\hat{P}_{c}=\mathbb{C}^{n-1} \times \mathbb{C}^{\times}$, we denote the components of this smooth map $\tilde{\mathcal{Z}}_{x}$ by $\tilde{z}$ and $\tilde{Z}$. Then it is straightforward to check that the following identities hold for all $\left(e^{\mathrm{i} \hat{q}}, \hat{p}\right) \in \mathbb{T}_{n} \times \overline{\mathfrak{C}}_{x}$ :

$$
\begin{gathered}
\hat{\zeta}(x, \tilde{z}(x, \hat{q}, \hat{p}))=\tau_{(x)} \zeta(x, \hat{p}) \tau_{(x)}^{-1}, \\
\hat{\theta}(x, \tilde{z}(x, \hat{q}, \hat{p}))=\tau_{(x)} \theta(x, \hat{p}) \tilde{\tau}_{(x)}^{-1}, \\
\Delta(x, \tilde{z}(x, \hat{q}, \hat{p}), \tilde{Z}(x, \hat{q}, \hat{p}))=\tau \tilde{\tau}_{(x)}^{-1} e^{-\hat{p}},
\end{gathered}
$$

where $\tau=\aleph\left(x, e^{\mathrm{i} \hat{q}}\right)$ as in (5.65),$\tau_{(x)}$ is given by (5.45), and for every $\tau \in \mathbb{T}_{n}$ we employ

$$
\tilde{\tau}_{(x)}:=\operatorname{diag}\left(1, \tau_{2}, \ldots, \tau_{n}\right) \quad \text { if } \quad x>0, \quad \tilde{\tau}_{(x)}:=\operatorname{diag}\left(\tau_{1}, \ldots, \tau_{n-1}, 1\right) \quad \text { if } \quad x<0 .
$$

Recall that the orthogonal matrices $\theta(x, \hat{p}), \zeta(x, \hat{p})$ were defined in Lemma 5.3 and 5.4 , respectively, and the matrices $\hat{\zeta}(x, z)$ and $\hat{\theta}(x, z)$ were introduced in Definition 3.3. The surjectivity of the map $\tilde{\mathcal{Z}}_{x}: \mathbb{T}_{n} \times \overline{\mathfrak{C}}_{x} \rightarrow \mathbb{C}^{n-1} \times \mathbb{C}^{\times}$now implies the unitarity of the matrices $\hat{\zeta}(x, z)$ and $\hat{\theta}(x, z)$ for every $x \neq 0$ and $z \in \mathbb{C}^{n-1}$.

The above identities give rise to the relation

$$
\left(\hat{\mathcal{I}} \circ \tilde{\mathcal{Z}}_{x}\right)(\hat{q}, \hat{p})=K_{x}\left(\mathbf{1}_{n}, \aleph\left(x, e^{\mathrm{i} \hat{q}}\right), \hat{p}\right), \quad \forall e^{\mathrm{i} \hat{q}} \in \mathbb{T}_{n}, \quad \forall \hat{p} \in \overline{\mathfrak{C}}_{x}
$$

where the map $\hat{\mathcal{I}}$ was defined in Eq. (3.42) and $K_{x}$ in Eq. (5.46). This implies by Lemma 5.5 and the surjectivity of the maps $\tilde{\mathcal{Z}}_{x}, \aleph(x,$.$) that every point of the constraint-manifold F_{\nu(x)}=$ $\Lambda^{-1}(\nu(x))$ can be written as $h \triangleright \hat{\mathcal{I}}(z, Z)$ with some $h \in G_{v(x)}$ and some $(z, Z) \in \mathbb{C}^{n-1} \times \mathbb{C}^{\times}$. In particular, the image of $\hat{\mathcal{I}}$ intersects every gauge orbit in $F_{\nu(x)}$.

Next, we wish to show that

$$
\hat{\mathcal{I}}^{*} \omega_{+}=\hat{\omega}_{c} .
$$

Referring to (5.69), the restriction of the relation (5.86) to $\mathbb{T}_{n} \times \mathfrak{C}_{x}$ can be expressed as

$$
k_{x}=\hat{\mathcal{I}} \circ \mathcal{Z}_{x} .
$$

By Eqs. (3.30) and (5.70), we have

$$
\mathcal{Z}_{x}^{*} \hat{\omega}_{c}=\hat{\omega}=k_{x}^{*} \omega_{+}=\mathcal{Z}_{x}^{*} \hat{\mathcal{I}}^{*} \omega_{+} .
$$


We recall from Remark 3.5 that the map $\mathcal{Z}_{x}$ yields a diffeomorphism between $\hat{P}$ and the dense open submanifold $\hat{P}_{c}^{0}$ of $\hat{P}_{c}$ given by

$$
\hat{P}_{c}^{0}:=\left\{(z, Z) \in \mathbb{C}^{n-1} \times \mathbb{C}^{\times} \mid z_{j} \neq 0, \forall j=1, \ldots, n-1\right\} .
$$

We thus see from Eq. (5.89) that the pull-back $\hat{\mathcal{I}}^{*} \omega_{+}$and the form $\hat{\omega}_{c}$ coincide everywhere on the dense open subset $\hat{P}_{c}^{0} \subset \hat{P}_{c}$. Therefore, by smoothness, they coincide everywhere on $\hat{P}_{c}$.

For $h \in G_{v(x)}$ and $(z, Z),\left(z^{\prime}, Z^{\prime}\right) \in \hat{P}_{c}$, we now prove the following implication:

$$
h \triangleright \hat{\mathcal{I}}(z, Z)=\hat{\mathcal{I}}\left(z^{\prime}, Z^{\prime}\right) \Longrightarrow h=\mathbf{1}_{n}, \quad z=z^{\prime}, \quad Z=Z^{\prime} .
$$

We present the argument in detail for the case $x>0$ and leave to the reader the analogous case $x<0$. The assumption on the left hand side of (5.91) entails that $h \triangleright \hat{\mathcal{I}}(z, Z)$ and $\hat{\mathcal{I}}\left(z^{\prime}, Z^{\prime}\right)$ have the same matrix $e^{-\hat{p}}$ in the modified Cartan decomposition (5.1). By means of the formulae (3.40), (3.41) and (3.42), this gives

$$
\Delta(x, z,|Z|)=\Delta\left(x, z^{\prime},\left|Z^{\prime}\right|\right)
$$

whence

$$
\left|Z^{\prime}\right|=|Z|, \quad\left|z_{i}^{\prime}\right|=\left|z_{i}\right|, \quad \forall i=1, \ldots, n-1 .
$$

Define the diagonal matrix $\Upsilon=\operatorname{diag}\left(\Upsilon_{1}, \ldots, \Upsilon_{n-1}, 1\right)$ by setting $\Upsilon_{j}:=\frac{z_{j}^{\prime}}{z_{j}}$ if $\left|z_{j}\right|=\left|z_{j}^{\prime}\right| \neq 0$ and $\Upsilon_{j}:=1$ otherwise. The inspection of the formulae (3.36), (3.37) and (3.38) together with Eq. (5.93) leads to the following equalities:

$$
\Upsilon \hat{\zeta}(x, z) \Upsilon^{-1}=\hat{\zeta}\left(x, z^{\prime}\right), \quad \Upsilon \hat{\theta}(x, z) \hat{\Upsilon}^{-1}=\hat{\theta}\left(x, z^{\prime}\right)
$$

where $\hat{\Upsilon}:=\operatorname{diag}\left(1, \Upsilon_{1}, \ldots, \Upsilon_{n-1}\right)$. Furthermore, the equality

$$
(h \triangleright \hat{\mathcal{I}}(z, Z))(h \triangleright \hat{\mathcal{I}}(z, Z))^{\dagger}=\hat{\mathcal{I}}\left(z^{\prime}, Z^{\prime}\right) \hat{\mathcal{I}}\left(z^{\prime}, Z^{\prime}\right)^{\dagger}
$$

implies

$$
h \kappa_{L}(x) \hat{\zeta}(x, z)^{-1} \Delta(x, z,|Z|)^{2} \hat{\zeta}(x, z) \kappa_{L}(x)^{-1} h^{-1}=\kappa_{L}(x) \hat{\zeta}\left(x, z^{\prime}\right)^{-1} \Delta(x, z,|Z|)^{2} \hat{\zeta}\left(x, z^{\prime}\right) \kappa_{L}(x)^{-1} .
$$

This gives

$$
\hat{\zeta}(x, z) \kappa_{L}(x)^{-1} h^{-1} \kappa_{L}(x)=\Gamma \hat{\zeta}\left(x, z^{\prime}\right),
$$

for some $\Gamma=\operatorname{diag}\left(\Gamma_{1}, \ldots, \Gamma_{n}\right) \in \mathbb{T}_{n}$ and, consequently,

$$
\hat{\zeta}(x, z) \kappa_{L}(x)^{-1} v(x)=\hat{\zeta}(x, z) \kappa_{L}(x)^{-1} h^{-1} \kappa_{L}(x) \kappa_{L}(x)^{-1} v(x)=\Gamma \hat{\zeta}\left(x, z^{\prime}\right) \kappa_{L}(x)^{-1} v(x) .
$$

By using Eqs. (5.42), (3.34) and the first equation of (5.94), we find from (5.98) that

$$
\Gamma_{n}=1 \quad \text { and } \quad \Gamma_{j}^{-1}=\Upsilon_{j} \quad \forall j \in\{1, \ldots, n-1\} \quad \text { for which } \quad z_{j} \neq 0 .
$$

By multiplying Eq. (5.97) by $\Gamma^{-1}$ from the right and by using the first equation of (5.94), we then arrive at

$$
\hat{\zeta}(x, z) \kappa_{L}(x)^{-1} h^{-1} \kappa_{L}(x) \Gamma^{-1}=\hat{\zeta}(x, z),
$$


and thus

$$
\kappa_{L}(x)^{-1} h \kappa_{L}(x)=\Gamma^{-1} .
$$

Now we return to the equation on the left hand side of the implication (5.91) and, by using Eqs. (3.42), (5.94) and (5.101), we rewrite it as

$$
\begin{gathered}
{\left[h \kappa_{L}(x) \hat{\zeta}(x, z)^{-1}\right] \triangleright\left[\Delta(x, z, Z) \hat{\theta}(x, z)^{-1}\right]=\left[\kappa_{L}(x) \Gamma^{-1} \hat{\zeta}(x, z)^{-1}\right] \triangleright\left[\Delta(x, z, Z) \hat{\theta}(x, z)^{-1}\right]=} \\
{\left[\kappa_{L}(x) \Gamma^{-1} \hat{\zeta}(x, z)^{-1} \Gamma\right] \triangleright\left[\Delta(x, z, Z) \Gamma^{-1} \hat{\theta}(x, z)^{-1} \Gamma\right]=\left[\kappa_{L}(x) \hat{\zeta}\left(x, z^{\prime}\right)^{-1}\right] \triangleright\left[\Delta\left(x, z^{\prime}, Z^{\prime}\right) \hat{\theta}\left(x, z^{\prime}\right)^{-1}\right] .}
\end{gathered}
$$

We remark that $\Gamma^{-1} \hat{\zeta}(x, z)^{-1} \Gamma=\Upsilon \hat{\zeta}(x, z)^{-1} \Upsilon^{-1}=\hat{\zeta}\left(x, z^{\prime}\right)^{-1}$, and hence from Eq. (5.102) we obtain

$$
\Delta\left(x, z^{\prime}, Z^{\prime}\right) \hat{\theta}\left(x, z^{\prime}\right)^{-1}=\Delta(x, z, Z) \Gamma^{-1} \hat{\theta}(x, z)^{-1} \Gamma .
$$

Taking into account Eq. (5.94), this yields

$$
\Upsilon \hat{\theta}(x, z) \hat{\Upsilon}^{-1} \Delta\left(x, z^{\prime}, Z^{\prime}\right)^{-1}=\Gamma^{-1} \hat{\theta}(x, z) \Gamma \Delta(x, z, Z)^{-1} .
$$

The $(j, j+1)$ entries of the matrices on the two sides of Eq. (5.104) never vanish. Together with (5.99), the equality of these entries implies that $\Gamma=\mathbf{1}_{n}=\Upsilon^{-1}$. We conclude that $h=\mathbf{1}_{n}$ by (5.101) and $z^{\prime}=z$ by the definition of $\Upsilon$. Similarly, the $(n, 1)$ entry never vanishes in (5.104), and this gives $Z^{\prime}=Z$. The implication (5.91) is therefore proven.

It is clear from its formula (3.42) and Lemma 3.2 that $\hat{\mathcal{I}}: \hat{P}_{c} \rightarrow F_{\nu(x)}$ is a smooth map. We see from the implication (5.91) that the map $\hat{\mathcal{I}}$ is injective and its image intersects every gauge orbit at most in one point. Since we have shown also that the image of $\hat{\mathcal{I}}$ intersects every gauge orbit, we conclude that condition 2) of Lemma 3.1 is satisfied. Eq. (5.87) guarantees that condition 1) of the same lemma holds. In conclusion, $\hat{\mathcal{I}}$ is a global cross section and $\left(\hat{P}_{c}, \hat{\omega}_{c}\right)$ is a model of the reduced phase space. Q.E.D.

Proof of Theorem 3.4: We first observe from (15.86) and the definition of $\hat{\mathcal{L}}(2.60)$ that

$$
\left(\hat{\mathcal{L}} \circ \hat{\mathcal{I}} \circ \mathcal{Z}_{x}\right)(\hat{q}, \hat{p})=\hat{\mathcal{L}}\left(K_{x}\left(\mathbf{1}_{n}, \aleph\left(x, e^{\mathrm{i} \hat{q}}\right), \hat{p}\right)\right)=\Xi_{R}\left(K_{x}\left(\mathbf{1}_{n}, \aleph\left(x, e^{\mathrm{i} \hat{q}}\right), \hat{p}\right)\right), \quad \forall(\hat{q}, \hat{p}) \in \hat{P} .
$$

Using the notation $X \sim Y$ to signify that the matrices $X$ and $Y$ are similar, we then conclude from (2.35) and the formula (5.46) of $K_{x}$ that

$$
\Xi_{R}\left(K_{x}\left(\mathbf{1}_{n}, \aleph\left(x, e^{\mathrm{i} \hat{q}}\right), \hat{p}\right)\right) \sim \Xi_{R}\left(e^{-\hat{p}}\left(\aleph\left(x, e^{\mathrm{i} \hat{q}}\right)\right)_{(x)}^{-1} \aleph\left(x, e^{\mathrm{i} \hat{q}}\right) \theta(x, \hat{p})^{-1}\right)=\theta(x, \hat{p}) e^{\mathrm{i} \hat{q}} .
$$

To obtain the last equality, we used the identity (5.66). The similarity transformation that appears in (5.106) is by a unitary matrix that one can find explicitly from the above. On the other hand, one can directly check with the aid of the formula of $\theta(x, \hat{p})$ given in Lemma 5.3 that the dual Ruijsenaars-Schneider Lax matrix (1.3) can be rewritten as

$$
\hat{L}(\hat{q}, \hat{p})=\hat{\delta} \theta(x, \hat{p}) e^{\mathrm{i} \hat{q}} \hat{\delta}^{-1}
$$

with

$$
\hat{\delta}=\operatorname{diag}\left(\hat{\delta}_{1}, \hat{\delta}_{2}, \ldots, \hat{\delta}_{n}\right), \quad \hat{\delta}_{j}=\prod_{m \neq j}\left[\frac{\sinh \left(\hat{p}_{j}-\hat{p}_{m}+\frac{x}{2}\right)}{\sinh \left(\hat{p}_{j}-\hat{p}_{m}-\frac{x}{2}\right)}\right]^{\frac{1}{4}}, \quad \forall j=1, \ldots, n
$$


Therefore the matrices $\left(\hat{\mathcal{L}} \circ \hat{\mathcal{I}} \circ \mathcal{Z}_{x}\right)(\hat{q}, \hat{p})$ and $\hat{L}(\hat{q}, \hat{p})$ are both similar to the matrix $\theta(x, \hat{p}) e^{\mathrm{i} \hat{q}}$. Q.E.D.

The implication (5.91) also confirms that $G_{v(x)}$ acts freely on $F_{\nu(x)}$, as was already shown in [11. Here, some further clarifying remarks are in order, which will be referred to in Section 6 .

Remark 5.1. Let $F_{\nu(x)}^{0}$ be the image of $G_{v(x)} \times \mathbb{T}_{n} \times \mathfrak{C}_{x}$ by the map $K_{x}$ (5.46). It follows from the above that $F_{\nu(x)}^{0}$ is a dense open submanifold of $F_{\nu(x)}$, which is stable under the gauge group $G_{v(x)}$ and is diffeomorphic to $G_{v(x)} \times \mathbb{T}_{n} \times \mathfrak{C}_{x}$ by $K_{x}$. Hence $F_{\nu(x)}^{0} / G_{v(x)}$ is a dense open submanifold of the full reduced phase space $F_{\nu(x)} / G_{v(x)}$. The phase space $(\hat{P}, \hat{\omega})(1.4)$ is a model of this dense open submanifold. In fact, a corresponding cross section (in the sense of Remark 3.2 ) is provided by that map $k_{x}: \hat{P} \rightarrow F_{\nu(x)}^{0}$ (5.69) that satisfies $k_{x}=\hat{\mathcal{I}} \circ \mathcal{Z}_{x}$ (5.88) with the symplectic embedding $\mathcal{Z}_{x}: \hat{P} \rightarrow \hat{P}_{c}$ given by Definition 3.2 .

Remark 5.2. We can define the $\mathbb{R}^{n}$-valued smooth (even real-analytic) $G_{v(x)}$-invariant function $\hat{\pi}$ on $F_{\nu(x)}$ by setting $\hat{\pi}\left(K_{x}(h, \tau, \hat{p})\right):=\hat{p}$. More directly, $\hat{\pi} \simeq \operatorname{diag}\left(\hat{\pi}_{1}, \ldots, \hat{\pi}_{n}\right)$ can be characterized by the property

$$
K K^{\dagger} \sim e^{-2 \hat{\pi}(K)} \quad \text { with } \quad \hat{\pi}(K) \in \overline{\mathfrak{C}}_{0},
$$

where, as before, $\sim$ denotes similarity of matrices. Clearly, $\hat{\pi}$ induces a smooth function on the full reduced phase space and it has $\overline{\mathfrak{C}}_{x}$ as its range. From the perspective of the completed dual Ruijsenaars-Schneider system, $\hat{\pi}$ can be viewed as a $\overline{\mathfrak{C}}_{x}$-valued globally well-defined 'position variable' (which coincides with $\hat{p}$ on the phase space $\hat{P}$ ).

We explained in [1] how Theorems 3.1 and 3.2 imply the known integration algorithm for the time development of the position variable $q$ along the commuting flows of the system $(P, \omega, L)$. Now we present the analogous result about the 'dual position variable' $\hat{\pi}$ (5.109) along the commuting flows of the dual system characterized by Theorems 3.3 and 3.4.

Remark 5.3. Take a Hamiltonian $\hat{H}:=\Xi_{R}^{*} \phi, \phi \in C^{\infty}(G)^{G}$ and regard it as a function on the model $\left(\hat{P}_{c}, \hat{\omega}_{c}\right)$ of the reduced phase space. Choose also an initial value $(z(0), Z(0)) \in \hat{P}_{c}$. Directly from the definitions, the associated initial value $\hat{\pi}(0):=\hat{\pi}(\hat{\mathcal{I}}(z(0), Z(0)))$ is subject to

$$
e^{-\hat{\pi}(0)}=\Lambda_{L}\left(\Delta_{0}\right) \quad \text { with } \quad \Delta_{0}:=\Delta(x, z(0), Z(0)) .
$$

By combining Theorem 3.3 with Corollary 2.1, it is easy to obtain the following result:

$$
e^{-2 \hat{\pi}(t)} \sim e^{-2 \hat{\pi}(0)} \exp \left[2 \mathrm{i} t \mathbf{D} \phi\left(\hat{\theta}(x, z(0)) \Xi_{R}\left(\Delta_{0}\right)\right)\right] .
$$

Since (5.109) defines $\hat{\pi}$ also as a $\bar{G}$-invariant function on the double, (5.111) follows immediately from (2.54) by replacing the initial value $\hat{\mathcal{I}}(z(0), Z(0))$ (3.42) by $\Delta(x, z(0), Z(0)) \hat{\theta}(x, z(0))^{-1}$. Indeed, the respective solutions $K(t)(2.49)$ are $\bar{G}$-related and yield the same $\hat{\pi}(t)$.

If $(z(0), Z(0)) \in\left(\hat{P}_{c} \backslash \hat{P}_{c}^{0}\right)$ (see (5.90) $)$, then the flow (5.111) does not stay in this lower dimensional closed submanifold in general, which explains by Theorem 3.4 why the commuting dual flows are not complete on $(\hat{P}, \hat{\omega})$. On the other hand, if $(z(0), Z(0)) \in \hat{P}_{c}^{0}$ and $(\hat{q}(0), \hat{p}(0))$ is the corresponding initial value in $\hat{P}=\mathcal{Z}_{x}^{-1}\left(\hat{P}_{c}^{0}\right)$, then (5.111) can be rewritten as

$$
e^{-2 \hat{\pi}(t)} \sim e^{-2 \hat{p}(0)} \exp \left[2 \mathrm{i} t \mathbf{D} \phi\left(\theta(x, \hat{p}(0)) e^{\mathrm{i} \hat{q}(0)}\right)\right] .
$$


To further elaborate, let us consider $\phi(g)=\operatorname{tr}(\chi(g))+$ c.c. with some complex power series $\chi$, for which $\mathbf{D} \phi(g)=\psi(g)-(\psi(g))^{\dagger}$ with $\psi(z):=z \chi^{\prime}(z)$ as was mentioned in (2.59). For $\psi(z)=\sum_{k=1}^{\infty} \psi_{k} z^{k}$, let us define $\tilde{\psi}(z):=\sum_{k=1}^{\infty} \bar{\psi}_{k} z^{k}$, where $\bar{\psi}_{k}$ is the complex conjugate of $\psi_{k}$. Then, by using (5.107), we can rewrite (5.112) equivalently as

$$
e^{-2 \hat{\pi}(t)} \sim e^{-2 \hat{p}(0)} \exp \left[2 \mathrm{i} t\left(\psi\left(\hat{L}_{0}\right)-\tilde{\psi}\left(\hat{L}_{0}^{-1}\right)\right)\right] \quad \text { with } \quad \hat{L}_{0}:=\hat{L}(\hat{q}(0), \hat{p}(0)) .
$$

These formulas for the flows were obtained previously in [30]. Our geometric picture renders their derivation essentially obvious.

\section{Discussion}

In this section, we summarize our construction in terms of diagrams of maps and explain the connection with the related results in [30]. Then we conclude and comment on open problems.

Our main arguments were concerned with the following diagram:

$$
\begin{array}{cccc}
P & \stackrel{\mathcal{I}}{\longrightarrow} & F_{\nu(x)} / G_{v(x)} \\
\mathcal{R} \downarrow & & \downarrow \text { id } \\
\hat{P}_{c} & \stackrel{\pi \circ \hat{\mathcal{I}}}{\longrightarrow} & F_{\nu(x)} / G_{v(x)}
\end{array}
$$

The maps $\mathcal{I}$ and $\pi \circ \hat{\mathcal{I}}$ are symplectomorphisms as claimed by Theorems 3.1 and 3.3. The map $\mathcal{R}$ is defined as the symplectomorphism that makes this diagram commute. We can add the phase space $(\hat{P}, \hat{\omega})(\underline{1.4})$ to (6.1) by using the embedding $\mathcal{Z}_{x}$ of Definition 3.2 and restrictions to the relevant dense open submanifolds. This leads to the second diagram:

$$
\begin{array}{ccccc}
P^{0} & \stackrel{\mathrm{id}}{\longrightarrow} & P^{0} & \stackrel{\mathcal{I}^{0}}{\longrightarrow} & F_{\nu(x)}^{0} / G_{v(x)} \\
\mathcal{R}^{0} \downarrow & & \mathcal{R}_{c}^{0} \downarrow & & \downarrow \text { id } \\
\hat{P} & \stackrel{\mathcal{Z}_{x}}{\longrightarrow} & \hat{P}_{c}^{0} & \stackrel{\pi \circ \hat{\mathcal{I}}^{0}}{\longrightarrow} & F_{\nu(x)}^{0} / G_{v(x)}
\end{array}
$$

Here, $P^{0} \subset P$ and $\hat{P}_{c}^{0} \subset \hat{P}_{c}$ are the dense open submanifolds symplectomorphic to the dense open submanifold $F_{\nu(x)}^{0} / G_{v(x)}$ of the reduced phase space, for which $\hat{\pi}$ (5.109) varies in $\mathfrak{C}_{x}$ (3.26). (See also (5.90) and Remark 5.1.) Whenever appropriate, the maps in (6.2) are obtained as the restrictions of those in (6.1). The map $\mathcal{R}^{0}$ is defined by the commutativity of the diagram (6.2). The symplectic forms $\omega(1.2)$ on $P, \hat{\omega}_{c}(3.27)$ on $\hat{P}_{c}$, and the reduced symplectic form on $F_{\nu(x)} / G_{v(x)}$ induce symplectic forms on the respective dense open submanifolds. Then all the maps $\mathcal{I}^{0}, \pi \circ \hat{\mathcal{I}}^{0}, \mathcal{R}_{c}^{0}, \mathcal{Z}_{x}$ and $\mathcal{R}^{0}$ are symplectomorphisms.

The maps $\mathcal{R}$ and $\mathcal{R}^{0}$ reproduce the 'complete' and the 'restricted' duality symplectomorphisms (alias action-angle maps) originally obtained by Ruijsenaars in [30] by means of direct arguments. In order to confirm this, we now present a useful consequence of our construction. 
Lemma 6.1. Consider a point $(q, p) \in P^{0}$ and its image $(\hat{q}, \hat{p}) \in \hat{P}$ by the symplectomorphism $\mathcal{R}^{0}$ defined by (6.2):

$$
\mathcal{R}^{0}(q, p)=(\hat{q}, \hat{p}),
$$

where $(q, p)$ parametrizes some point $\left(e^{2 \mathrm{i} q}, p\right)$ of the covering space $T^{*} \mathbb{T}_{n}^{0}$ (3.17) of $P$ (3.16). Then the Lax matrices $L(q, p)$ (1.1) and $\hat{L}(\hat{q}, \hat{p})$ (1.3) satisfy the similarity relations

$$
L(q, p) \sim e^{2 \hat{p}} \quad \text { and } \quad \hat{L}(\hat{q}, \hat{p}) \sim e^{2 \mathrm{i} q} .
$$

Proof. First, let us recall from Theorem 3.2 that $L(q, p) \sim(\mathcal{L} \circ \tilde{\mathcal{I}})\left(e^{2 \mathrm{i} q}, p\right)$. The definition (2.60) of the unreduced Lax matrix $\mathcal{L}$ gives $(\mathcal{L} \circ \tilde{\mathcal{I}})\left(e^{2 \mathrm{i} q}, p\right)=\tilde{\mathcal{I}}\left(e^{2 \mathrm{i} q}, p\right)^{-1}\left(\tilde{\mathcal{I}}\left(e^{2 \mathrm{i} q}, p\right)^{-1}\right)^{\dagger}$, and then we obtain

$\tilde{\mathcal{I}}\left(e^{2 \mathrm{i} q}, p\right)^{-1}\left(\tilde{\mathcal{I}}\left(e^{2 \mathrm{i} q}, p\right)^{-1}\right)^{\dagger} \sim\left(\tilde{\mathcal{I}}\left(e^{2 \mathrm{i} q}, p\right) \tilde{\mathcal{I}}\left(e^{2 \mathrm{i} q}, p\right)^{\dagger}\right)^{-1} \sim\left(\left(\left(\hat{\mathcal{I}} \circ \mathcal{Z}_{x}\right)(\hat{q}, \hat{p})\right)\left(\left(\hat{\mathcal{I}} \circ \mathcal{Z}_{x}\right)(\hat{q}, \hat{p})\right)^{\dagger}\right)^{-1} \sim e^{2 \hat{p}}$

Here we used that, because of $(\underline{6.3}), \tilde{\mathcal{I}}\left(e^{2 \mathrm{i} q}, p\right)$ and $\left(\hat{\mathcal{I}} \circ \mathcal{Z}_{x}\right)(\hat{q}, \hat{p})$ must lie on the same gauge orbit in $F_{\nu(x)}^{0}$, which implies the second similarity relation in (6.5) on account of the first relation in (2.35). The last relation in (6.5) follows from (5.109), since $\hat{\pi}\left(\hat{\mathcal{I}} \circ \mathcal{Z}_{x}(\hat{q}, \hat{p})\right)=\hat{p}$ holds on $\hat{P}$. Thus the first claim of (6.4) is proved. To prove the second claim of (6.4) , we notice from Theorem 3.4 together with the definition of $\hat{\mathcal{L}}$ in (2.60) and the second relation in (2.35) that

$$
\hat{L}(\hat{q}, \hat{p}) \sim\left(\hat{\mathcal{L}} \circ \hat{\mathcal{I}} \circ \mathcal{Z}_{x}\right)(\hat{q}, \hat{p}) \sim \Xi_{R}\left(\tilde{\mathcal{I}}\left(e^{2 \mathrm{i} q}, p\right)\right)=e^{2 \mathrm{i} q} .
$$

The last equality above is a direct consequence of the formula (4.18) of $\tilde{\mathcal{I}}\left(e^{2 \mathrm{i} q}, p\right)$. Q.E.D.

We see from (6.4) that our symplectomorphism $\mathcal{R}^{0}$ between the restricted phase spaces $P^{0}$ and $\hat{P}$ converts the action variables of the original system (1.1) into the particle-coordinates of the dual system (1.3), and vice versa. These relations completely characterize the map $\mathcal{R}^{0}$ and also hold for the restricted duality symplectomorphism constructed in [30]. Therefore the latter is indeed reproduced by our geometric construction. The complete duality symplectomorphism $\mathcal{R}$ of diagram (6.1) is also the same as the one obtained in [30], simply because the embedding $\mathcal{Z}_{x}: \hat{P} \rightarrow \hat{P}_{c}$ occurs in [30] as well and its image is dense in $\hat{P}_{c}$.

To conclude, we have shown in this paper that the duality symplectomorphism between the system $(P, \omega, L)(1.1)$ and the completion of the system $(\hat{P}, \hat{\omega}, \hat{L})(1.3)$ is nothing but the geometrically natural map between two models of the reduced phase space associated with a symplectic reduction of the Heisenberg double of $U(n)$. The character of the completion of the phase space $(\hat{P}, \hat{\omega})$ is thereby illuminated: $(\hat{P}, \hat{\omega})$ represents a dense open submanifold of the full reduced phase space wherein the reduced flows inherited from the free flows on the double are naturally complete. We have also seen that the reduction turns the two Abelian algebras $\mathcal{H}$ and $\hat{\mathcal{H}}(2.29)$ (spanned by the unreduced free Hamiltonians) into the Abelian algebras of the action and the particle-position variables of the system $(P, \omega, L)$, respectively, and the rôle of these algebras is exchanged when viewed from the perspective of the dual system. In addition, we

\footnotetext{
${ }^{4}$ To provide a short dictionary: our maps $\mathcal{R}^{0}$ and $\mathcal{R}$ reproduce the maps $\Phi$ and $\Phi^{\sharp}$ that feature in diagrams (1.67) and (1.74) in [30], respectively. The content of our Remark 5.3 is consistent with Theorem 5.7 in [30].
} 
obtained the Ruijsenaars-Schneider Lax matrices as well as the integration algorithms for their flows as easy by-products. The present article, together with the previous one [10], leave little doubt that all cases of the duality studied in [27, 29, 30] must permit analogous interpretation in terms of symplectic reduction of finite-dimensional integrable systems of group theoretic origin 5 . The details are not trivial and we plan to return to other examples elsewhere.

It is proper to mention at this point that although we expect that all cases of Ruijsenaars' duality can be treated by the reduction method, the direct approach also has its own advantages. For instance, it appears that the detailed analyses of the scattering behaviour performed in [27, 29, 30] cannot be simplified by group theoretic means.

It can be surmised from results in [12, 26] that the classical trigonometric $B C_{n}$ systems of van Diejen [36] must admit a derivation based on a reduction of the Heisenberg double of $U(2 n)$, which should produce the so far missing Lax matrices for these systems. We plan to elaborate this, building on the description of the free systems given in Section 2 of the present paper. The results collected there might prove to be useful in other studies in the reduction approach too, for example to obtain spin Ruijsenaars-Schneider systems.

Finally, it is pertinent to remark that the quantum mechanical analogue of Ruijsenaars' duality is the so-called bispectral property [6] and it is known that the quantum mechanical variants of the systems $(P, \omega, L)$ and $(\hat{P}, \hat{\omega}, \hat{L})$ form a bispectral pair [28]. This follows from explicit inspection of the eigenfunctions of the respective Hamiltonian operators. The eigenfunctions are provided by Macdonald polynomials [28, 37, 4], which also have close connections to quantum groups [9, 24, 21] and to double affine Hecke algebras [5, 25]. It would be interesting to understand the bispectral property, at least for certain (integer) values of the coupling constant, in terms of a quantum analogue of our classical reduction.

\section{A Regularity of the moment map value $\nu(x)$}

The purpose of this appendix is to demonstrate that $\nu(x)(3.10)$ is a regular value of the Poisson-Lie moment map $\Lambda$ associated with the quasi-adjoint action on $G L(n, \mathbb{C})$.

We first recall from [11] that every element $K \in G L(n, \mathbb{C})$ can be represented as

$$
K=g \triangleright\left(b T^{-1}\right) \quad \text { with } \quad g \in G=U(n), T \in \mathbb{T}_{n}, b \in B .
$$

It was also shown in [1] that every element of the constraint-manifold

$$
F_{\nu(x)}=\{K \in G L(n, \mathbb{C}) \mid \Lambda(K)=\nu(x)\}
$$

can be written as

$$
K=g \triangleright\left(\mathcal{N}(T) a T^{-1}\right) \quad \text { with } \quad g \in G_{\nu(x)}, T \in \mathcal{C}, a \in A .
$$

Here $\mathcal{C}$ is a subset of the regular elements $\mathbb{T}_{n}^{0} \subset \mathbb{T}_{n}$, such that $\mathcal{C}$ intersects every orbit of the permutation group $S(n)$ in $\mathbb{T}_{n}^{0}$ precisely in one point. $A<B$ is the group of diagonal matrices

\footnotetext{
${ }^{5}$ The question of possible dualities in the elliptic case is wide open. For the reduction approach to elliptic systems, see for example [2] and references therein.
} 
with positive real entries, and $\mathcal{N}(T)$ belongs to the subgroup $N<B$ of upper-triangular matrices with unit diagonal, given explicitly by the formula (4.1).

The formula (2.22) of $\Lambda$ gives

$$
\Lambda\left(b T^{-1}\right)=b T b^{-1} T^{-1} \in N, \quad \forall T \in \mathbb{T}_{n}, \forall b \in B .
$$

By using this together with the $U(n)$ equivariance of $\Lambda$ and (A.1), we easily obtain that $\operatorname{det} \Lambda(K)=1$ for all $K \in G L(n, \mathbb{C})$. In other words, $\Lambda$ takes its values in the normal subgroup $S B$ of $B$ consisting of elements of unit determinant.

Proposition A.1. The constant $\nu(x)($ (3.10) is a regular value of the Poisson-Lie moment map $\Lambda: G L(n, \mathbb{C}) \rightarrow S B$.

Proof. By the equivariance property of $\Lambda$ and (A.3), it is sufficient to show that the derivative (tangent) map

$$
\lambda_{K}:=T \Lambda(K): T_{K} G L(n, \mathbb{C}) \rightarrow T_{\nu(x)} S B
$$

is surjective at every point of $F_{\nu(x)}$ of the form

$$
K=\mathcal{N}(T) a T^{-1} \quad \text { with } \quad T \in \mathcal{C}, a \in A .
$$

Now the tangent space to $S B$ at any of its elements can be identified, as a vector space, with $s \mathcal{B}$ : the space of upper triangular, traceless matrices with real diagonal entries. This simply follows from the structure of $S B$ as a matrix group. Take an arbitrary $Y \in s \mathcal{B}$ and consider the tangent vector

$$
Y K \in T_{K} G L(n, \mathbb{C}),
$$

which is the velocity of the curve $e^{Y t} K$ at $t=0$. At $K$ of the form (A.6), the formula (A.4) leads to

$$
\lambda_{K}(Y K)=Y \nu(x)-\nu(x)\left(T Y T^{-1}\right) \in \operatorname{Lie}(N) \subset s \mathcal{B}, \quad \forall Y \in s \mathcal{B} .
$$

This equation implies that for any $Z \in \operatorname{Lie}(N)$ there exists a unique $Y \in \operatorname{Lie}(N)$ for which $\lambda_{K}(Y K)=Z$. To verify this, consider the principal gradation of $g l(n, \mathbb{C})$ by 'heights' and write accordingly

$$
Y=Y_{1}+Y_{2}+\cdots+Y_{n-1}, \quad Z=Z_{1}+Z_{2}+\cdots+Z_{n-1}, \quad \nu(x)=\mathbf{1}_{n}+\nu_{1}+\nu_{2}+\cdots+\nu_{n-1} .
$$

First, the grade 1 part of

$$
Y \nu(x)-\nu(x)\left(T Y T^{-1}\right)=Z
$$

reads

$$
Y_{1}-T Y_{1} T^{-1}=Z_{1}
$$

which determines $Y_{1}$ uniquely in terms of $Z_{1}$ and $T$ since $T$ is regular. Second, the grade 2 part of (A.10) reads

$$
Y_{2}-T Y_{2} T^{-1}+\left(Y_{1} \nu_{1}-\nu_{1} T Y_{1} T^{-1}\right)=Z_{2}
$$

which determines $Y_{2}$ uniquely in terms of $Z_{1}, Z_{2}$ and $T$. Obviously, this argument can be continued increasing the grade until the top grade, $(n-1)$, is reached. 
We have seen that the image of $\lambda_{K}($ A.5), at $K$ in $(\underline{A .6})$, contains $\operatorname{Lie}(N)$. Denoting by $s \mathcal{A}$ the space of diagonal elements of $s \mathcal{B}$, we have

$$
s \mathcal{B}=s \mathcal{A}+\operatorname{Lie}(N),
$$

and it remains to show that the $s \mathcal{A}$-projections of the elements in the image of $\lambda_{K} \operatorname{span} s \mathcal{A}$. For $W \in u(n)$, denote by $W \triangleright K$ the velocity of the curve $e^{t W} \triangleright K$ at $t=0$. Because the moment map is equivariant, we obtain

$$
\lambda_{K}(W \triangleright K)=\operatorname{dress}_{W} \nu(x) \equiv \nu(x)\left(\nu^{-1}(x) W \nu(x)\right)_{\mathcal{B}},
$$

where we use the unique decomposition

$$
X=X_{\mathcal{B}}+X_{u(n)}, \quad \forall X \in g l(n, \mathbb{C}) .
$$

Note from (3.10) that

$$
\nu(x)=\mathbf{1}_{n}+\left(2 \sinh \frac{x}{2}\right) I_{1}+\cdots, \quad \text { where } \quad I_{1}=\sum_{j=1}^{n-1} E_{j, j+1}
$$

and the dots stand for the higher grade parts of $\nu(x)$. Let us now take an element $W \in u(n)$ of the form

$$
W=W_{-1}-\left(W_{-1}\right)^{\dagger}, \quad W_{-1}=\sum_{k=1}^{n-1} \xi_{k} E_{k+1, k}
$$

with some real numbers $\xi_{k}$. Then we easily obtain

$$
\left.\left(\nu^{-1}(x) W \nu(x)\right)\right)_{\mathcal{B}}=-\left(2 \sinh \frac{x}{2}\right)\left[I_{1}, W_{-1}\right]+\text { non-zero grade contributions. }
$$

Therefore, from (A.13), in this case we get the $s \mathcal{A}$-projection

$$
\pi_{s \mathcal{A}}\left(\lambda_{K}(W \triangleright K)\right)=-2\left(\sinh \frac{x}{2}\right) \sum_{k=1}^{n-1} \xi_{k}\left(E_{k, k}-E_{k+1, k+1}\right) .
$$

These elements span $s \mathcal{A}$. Hence we conclude that $\lambda_{K}($ A.5 $)$ is surjective. Q.E.D.

Remark A.1. It was shown in [11] (page 135) that the 'constituents' $T \in \mathcal{C}, a \in A$ and $g \in G_{\nu(x)}$ that appear in (A.3) are uniquely determined by $K \in F_{\nu(x)}$ up to the obvious freedom of multiplying $g$ by an element from the central $U(1)$ subgroup of $U(n)$. This means, in particular, that $\bar{G}_{\nu(x)}($ (3.13) $)$ acts freely on $F_{\nu(x)}$. To avoid possible confusion, we point out that the set $\mathcal{C}$ cannot be taken as a globally smooth submanifold of $\mathbb{T}_{n}^{0}$. This follows from the non-triviality of the $S(n)$-bundle $\mathbb{T}_{n}^{0} \rightarrow Q(n)$; see the subsequent appendix. 


\section{B The structure of the configuration space $Q(n)$}

In this appendix we deal with some features of the non-trivial manifold,

$$
Q(n)=\mathbb{T}_{n}^{0} / S(n)
$$

which is the configuration space of $n$ indistinguishable non-coinciding 'point-particles' moving on the circle. We shall expound the following statements:

1. The principal $S(n)$-bundle $\mathbb{T}_{n}^{0} \rightarrow Q(n)$ is topologically non-trivial.

2. The manifold $Q(n)$ is orientable if and only if $n$ is odd.

3. $Q(n)$ cannot be separated into the Cartesian product of a 'center of mass circle' and a 'space of relative positions'.

Note that the first statement can be extracted also from [30] and the second one generalizes the result of Leinaas and Myrheim [19] who showed that $Q(2)$ is the open Möbius strip.

To begin, we remark that the elements of the manifold $\mathbb{T}_{n}^{0}$ can be viewed as the configurations of $n$ distinguished non-coinciding points on a circle. We can label those points by integers $1, \ldots, n$ according to their registration on the diagonal in the matrix realization of $\mathbb{T}_{n}^{0}$. Consider an element $E$ of $\mathbb{T}_{n}^{0}$ and its $S(n)$-action image $\sigma(E)$ under any permutation $\sigma \in S(n)$ that changes the cyclic order of the distinguished points on the circle. It is evident that we cannot smoothly connect the element $E$ with its image $\sigma(E)$ without violating the condition of non-coincidence. This means that the element $E$ and its image $\sigma(E)$ live respectively in two different connected components of $T_{n}^{0}$. On the other hand, it is not difficult to see that the permutations respecting the cyclic order (i.e. the cyclic permutations) can be realized smoothly, which means that the element $E$ and its image under the cyclic permutation live on the same connected component in $\mathbb{T}_{n}^{0}$. Thus the connected components of $\mathbb{T}_{n}^{0}$ correspond to the coset space $S(n) / \mathbb{Z}_{n}$. In particular, this implies that the principal $S(n)$-bundle $\mathbb{T}_{n}^{0} \rightarrow Q(n)$ is topologically non-trivial, since if it were trivial then the element $E$ and its image $\sigma(E)$ would live on different connected components for each permutation $\sigma$ not equal to the identity.

Consider the connected component $K$ of $\mathbb{T}_{n}^{0}$ characterized by the requirement that the order of the labels $1<2<\ldots<n$ conforms with the cyclic order in which the distinguished points appear on the circle. We know from the preceding paragraph that the manifold $K$ is a topologically non-trivial $\mathbb{Z}_{n}$-covering of the manifold $Q(n)$. In fact, the non-triviality of the $\mathbb{Z}_{n}$-bundle $K \rightarrow Q(n)$ follows from the connectedness of $K$, which also implies that $Q(n)$ is connected. Now we remark that $K$ can be identified as the Cartesian product of the unit circle, on which the distinguished points live, and of the $(n-1)$-dimensional open simplex $\operatorname{Simp}_{n-1}$ given by

$$
\operatorname{Simp}_{n-1}:=\left\{\delta \in \mathbb{R}^{n-1} \mid \delta_{j}>0, \quad \sum_{j=1}^{n-1} \delta_{j}<2 \pi\right\} .
$$

Indeed, the point $z$ on the unit circle corresponds to the position of the distinguished point 1 , the number $0<\delta_{1}<2 \pi$ is the angle between the points 1 and $2, \delta_{2}$ between the points 2 and 3 
etc. The generator $\Gamma$ of the cyclic group $\mathbb{Z}_{n}$ is the cyclic permutation $(123 \ldots n) \rightarrow(n 12 \ldots n-1)$. It is very easy to find the action of $\Gamma$ on $K=U(1) \times \operatorname{Simp}_{n-1}$ :

$$
\Gamma\left(z, \delta_{1}, \delta_{2}, \ldots, \delta_{n-2}, \delta_{n-1}\right)=\left(z e^{\mathrm{i} \delta_{1}}, \delta_{2}, \delta_{3}, \ldots, \delta_{n-1}, 2 \pi-\sum_{j=1}^{n-1} \delta_{j}\right)
$$

A simple calculation of the Jacobian gives the transformation law of the volume form on $K$ under the $\Gamma$-transformation:

$$
\Gamma^{*}\left(\mathrm{i} z^{-1} d z \wedge d \delta_{1} \wedge \ldots \wedge d \delta_{n-1}\right)=(-1)^{n+1} \mathrm{i} z^{-1} d z \wedge d \delta_{1} \wedge \ldots \wedge d \delta_{n-1} .
$$

We observe that for $n$ odd the volume form is $\mathbb{Z}_{n}$ invariant and therefore it descends to an everywhere non-vanishing $n$-form on the quotient $K / \mathbb{Z}_{n}=Q(n)$. This means that for $n$ odd $Q(n)$ is orientable. Suppose now that there is also an everywhere non-vanishing $n$-form $\alpha$ on $Q(n)$ for $n$ even. Its pull-back $\tilde{\alpha}$ to $K$ must be given by a formula

$$
\tilde{\alpha}=f\left(z, \delta_{1}, \ldots, \delta_{n-1}\right) \mathrm{i} z^{-1} d z \wedge d \delta_{1} \wedge \ldots \wedge d \delta_{n-1},
$$

where $f\left(z, \delta_{1}, \ldots, \delta_{n-1}\right)$ is a smooth and everywhere non-vanishing real function on $K$. On the other hand, it must hold also that

$$
\Gamma^{*}(\tilde{\alpha})=\tilde{\alpha},
$$

because otherwise $\tilde{\alpha}$ would not be the pull-back of $\alpha$ from $Q(n)$. For $n$ even, the condition (B.6) says that

$$
f\left(z e^{\mathrm{i} \delta_{1}}, \delta_{2}, \delta_{3}, \ldots, \delta_{n-1}, 2 \pi-\sum_{j=1}^{n-1} \delta_{j}\right)=-f\left(z, \delta_{1}, \delta_{2}, \ldots, \delta_{n-2}, \delta_{n-1}\right) .
$$

Since $f$ changes sign if we move from a point on $K$ to its $\Gamma$ image and $f$ is smooth, it must vanish somewhere on $K$, which is in contradiction with the orientability of $Q(n)$ for $n$ even.

So far we have proved the first two statements displayed at the beginning. For the last statement, we notice that the permutation action (B.3) of $\mathbb{Z}_{n}$ on $K$ commutes with the obvious action of $U(1)$ on $K$ given by

$$
u\left(z, \delta_{1}, \delta_{2}, \ldots, \delta_{n-2}, \delta_{n-1}\right)=\left(u z, \delta_{1}, \delta_{2}, \ldots, \delta_{n-2}, \delta_{n-1}\right), \quad u \in U(1) .
$$

Therefore this $U(1)$ action descends to $Q(n)$. In specific systems, e.g. in the trigonometric Ruijsenaars-Schneider and Sutherland systems, the $U(1)$ action on $Q(n)$ just described can be interpreted as global rotation symmetry. Suppose that one tries to separate $Q(n)$ into the Cartesian product of a 'center of mass circle' and some manifold of 'relative positions', say $R(n-1)$. A reasonable definition of such separation requires the existence of a product representation, $Q(n) \simeq U(1) \times R(n-1)$, such that $U(1)$ acts only on $U(1)$ and not on $R(n-1)$. One might contemplate actions $\mathcal{A}_{u}$ of the global rotations on $U(1) \times R(n-1)$ defined by

$$
\mathcal{A}_{u}(w, r):=\left(u^{k} w, r\right), \quad \forall(w, r) \in U(1) \times R(n-1),
$$

where $k$ is a fixed, non-zero integer. The choice $k=1$ might appear the most natural, while $k=n$ corresponds to taking the hypothetical center of mass as the product of the $n$ points on 
the unit circle. However, we now show that neither of these separations of $Q(n)$ exists. Indeed, if we consider a point $\left(z, \delta_{1}, \ldots, \delta_{n-1}\right)$ in $K$ such that $\delta_{j}=\frac{2 \pi}{n}$ for every $j$, then we obtain

$$
e^{\frac{2 \pi \mathrm{i}}{n}}\left(z, \frac{2 \pi}{n}, \frac{2 \pi}{n}, \ldots, \frac{2 \pi}{n}\right)=\left(z e^{\frac{2 \pi \mathrm{i}}{n}}, \frac{2 \pi}{n}, \frac{2 \pi}{n}, \ldots, \frac{2 \pi}{n}\right)=\Gamma\left(z, \frac{2 \pi}{n}, \frac{2 \pi}{n}, \ldots, \frac{2 \pi}{n}\right) .
$$

Hence the action of $e^{\frac{2 \pi \mathrm{i}}{n}} \in U(1)$ leaves invariant the point of $Q(n)$ covered by $\left(z, \frac{2 \pi}{n}, \frac{2 \pi}{n}, \ldots, \frac{2 \pi}{n}\right) \in$ $K$. On the other hand, the isotropy group of the generic elements of $Q(n)$ is trivial under the natural $U(1)$ action. In contrast, under the 'separated action' (B.9) all points have the same isotropy group, $\mathbb{Z}_{k}$. This contradiction implies the validity of our third statement.

Acknowledgements. We wish to thank Ian Marshall for useful comments on the manuscript. L.F. was partially supported by the Hungarian Scientific Research Fund (OTKA) under the grant K 77400.

\section{References}

[1] Alekseev, A.Yu., Malkin, A.Z.: Symplectic structures associated to Lie-Poisson groups. Commun. Math. Phys. 162, 147-174 (1994)

[2] Arutyunov, G.E., Chekhov, L.O., Frolov, S.A.: Quantum dynamical $R$-matrices. In: Moscow Seminar in Mathematical Physics, AMS Transl. Ser. 2, Vol. 191, 1999, pp. 132

[3] Calogero, F.: Solution of the one-dimensional $N$-body problem with quadratic and/or inversely quadratic pair potentials. J. Math. Phys. 12, 419-436 (1971)

[4] Chalykh, O.: Macdonald polynomials and algebraic integrability. Adv. Math. 166, 193-259 (2002)

[5] Cherednik, I.: Double Affine Hecke Algebras. Cambridge University Press, 2005.

[6] Duistermaat, J.J., Grünbaum, F.A.: Differential equations in the spectral parameter. Commun. Math. Phys. 103, 177-240 (1986)

[7] Duistermaat, J.J., Kolk, J.A.C.: Lie Groups. Springer, 2000.

[8] Etingof, P.: Calogero-Moser Systems and Representation Theory. European Mathematical Society, 2007.

[9] Etingof, P.I., Kirillov Jr., A.A.: Macdonald's polynomials and representations of quantum groups. Math. Res. Lett. 1, 279-294 (1994)

[10] Fehér, L., Klimčík, C.: On the duality between the hyperbolic Sutherland and the rational Ruijsenaars-Schneider models. J. Phys. A: Math. Theor. 42, 185202 (2009) 
[11] Fehér, L., Klimčík, C.: Poisson-Lie generalization of the Kazhdan-Kostant-Sternberg reduction. Lett. Math. Phys. 87, 125-138 (2009)

[12] Fehér, L., Pusztai, B.G.: A class of Calogero type reductions of free motion on a simple Lie group. Lett. Math. Phys. 79, 263-277 (2007)

[13] Fock, V., Gorsky, A., Nekrasov, N., Rubtsov, V.: Duality in integrable systems and gauge theories. JHEP 07, 028 (2000)

[14] Fock, V.V., Rosly, A.A.: Poisson structure on moduli of flat connections on Riemann surfaces and the r-matrix. In: Moscow Seminar in Mathematical Physics, AMS Transl. Ser. 2, Vol. 191, 1999, pp. 67-86

[15] Gorsky, A., Nekrasov, N.: Relativistic Calogero-Moser model as gauged WZW theory. Nucl. Phys. B 436, 582-608 (1995)

[16] Kazhdan, D., Kostant, B., Sternberg, S.: Hamiltonian group actions and dynamical systems of Calogero type. Comm. Pure Appl. Math. XXXI, 481-507 (1978)

[17] Klimčík, C.: Quasitriangular WZW model. Rev. Math. Phys. 16, 679-808 (2004)

[18] Klimčík, C.: On moment maps associated to a twisted Heisenberg double. Rev. Math. Phys. 18, 781-821 (2006)

[19] Leinaas, J.M., Myrheim, J.: On the theory of identical particles. Nuovo Cim. B 37, 1-23 (1977)

[20] Lu, J.-H.: Moment maps and reduction of Poisson actions. In: Proc. Sem. Sud-Rhodanien de Geometrie à Berkeley (1989), Springer-Verlag MSRI Publ., Vol. 20, 1991, pp. 209-226

[21] Mimachi, K: Macdonald's operator from the center of the quantized universal enveloping algebra $U_{q}(g l(N))$. Int. Math. Res. Notices IMRN 1994 no.10, 415-424 (1994)

[22] Moser, J.: Three integrable Hamiltonian systems connected with isospectral deformations. Adv. Math. 16, 197-220 (1975)

[23] Nekrasov, N.: Infinite-dimensional algebras, many-body systems and gauge theories. In: Moscow Seminar in Mathematical Physics, AMS Transl. Ser. 2, Vol. 191, 1999, pp. 263-299

[24] Noumi, M.: Macdonald's symmetric polynomials as zonal spherical functions on some quantum homogeneous spaces. Adv. Math. 123, 16-77 (1996)

[25] Oblomkov, A.A.: Double affine Hecke algebras and Calogero-Moser spaces. Represent. Theory 8, 243266 (2004)

[26] Oblomkov, A.A., Stokman, J.V.: Vector valued spherical functions and MacdonaldKoornwinder polynomials. Compos. Math. 141, 1310-1350 (2005)

[27] Ruijsenaars, S.N.M.: Action-angle maps and scattering theory for some finite-dimensional integrable systems I. The pure soliton case. Commun. Math. Phys. 115, 127-165 (1988) 
[28] Ruijsenaars, S.N.M.: Finite-dimensional soliton systems. In: Integrable and Superintegrable Systems, ed. B. Kupershmidt, World Scientific, 1990, pp. 165-206

[29] Ruijsenaars, S.N.M.: Action-angle maps and scattering theory for some finite-dimensional integrable systems II. Solitons, antisolitons and their bound states. Publ. RIMS 30, 865$1008(1994)$

[30] Ruijsenaars, S.N.M.: Action-angle maps and scattering theory for some finite-dimensional integrable systems III. Sutherland type systems and their duals. Publ. RIMS 31, 247-353 (1995)

[31] Ruijsenaars, S.N.M.: Systems of Calogero-Moser type. In: Proceedings of the 1994 CRMBanff Summer School 'Particles and Fields', Springer, 1999, pp. 251-352

[32] Ruijsenaars, S.N.M., Schneider, H.: A new class of integrable models and their relation to solitons. Ann. Phys. (N.Y.) 170, 370-405 (1986)

[33] Semenov-Tian-Shansky, M.A.: Dressing transformations and Poisson groups actions. Publ. RIMS 21, 1237-1260 (1985)

[34] Sutherland, B.: Exact results for a quantum many-body problem in one dimension. Phys. Rev. A4, 2019-2021 (1971)

[35] Sutherland, B.: Beautiful Models, World Scientific, 2004.

[36] van Diejen, J.F.: Deformations of Calogero-Moser systems and finite Toda chains. Theor. Math. Phys. 99, 549-554 (1994)

[37] van Diejen, J.F.: On the diagonalization of difference Ruijsenaars-Schneider systems. In: Symmetries and Integrability of Difference Equations, Amer. Math. Soc., 1996, pp. 79-89

[38] Zakrzewski, S.: Free motion on the Poisson $S U(N)$ group. J. Phys. A: Math. Gen. 30, 6535-6543 (1997) 